\title{
A variational multiscale finite element method for monolithic ALE computations of shock hydrodynamics using nodal elements
}

\author{
X. Zeng ${ }^{\mathrm{a}, *}$, G. Scovazzi ${ }^{\mathrm{a}}$ \\ ${ }^{a}$ Department of Civil and Environmental Engineering, Duke University, Durham, North Carolina 27708
}

\begin{abstract}
We present a monolithic arbitrary Lagrangian-Eulerian (ALE) finite element method for computing highly transient flows with strong shocks. We use a variational multiscale (VMS) approach to stabilize a piecewise-linear Galerkin formulation of the equations of compressible flows, and an entropy artificial viscosity to capture strong solution discontinuities. Our work demonstrates the feasibility of VMS methods for highly transient shock flows, an area of research for which the VMS literature is extremely scarce.

In addition, the proposed monolithic ALE method is an alternative to the more commonly used Lagrangian+remap methods, in which, at each time step, a Lagrangian computation is followed by mesh smoothing and remap (conservative solution interpolation). Lagrangian+remap methods are the methods of choice in shock hydrodynamics computations because they provide nearly optimal mesh resolution in proximity of shock fronts. However, Lagrangian+remap methods are not well suited for imposing inflow and outflow boundary conditions. These issues offer an additional motivation for the proposed approach, in which we first perform the mesh motion, and then the flow computations using the monolithic ALE framework.

The proposed method is second-order accurate and stable, as demonstrated by extensive numerical examples in two and three space dimensions.
\end{abstract}

Key words: Variational multiscale finite element method, nodal finite elements, entropy viscosity, monolithic arbitrary Lagrangian-Eulerian, shock hydrodynamics

\section{Introduction}

Shock hydrodynamics is characterized by very unsteady flows and the presence of strong discontinuities. Because of their excellent resolution near shocks, Lagrangian methods have been initially preferred in computations. However, pure Lagrangian methods are not feasible in most practical cases, since the naturally arising vorticity in the fluid induces mesh tangling. Hence, Lagrangian methods, per se, cannot be applied successfully in general computations, other than specific cases, such as one-dimensional or radially symmetric flows.

To overcome this issue, common shock hydrodynamics algorithms (hydrocodes, in short) combine a Lagrangian computation with a mesh smoothing procedure and a data-transfer stage at each time step. Examples include the next non-exhaustive list of publications in this area [1-15].

For the same total number of computational unknowns, Lagrangian+remap methods have significantly superior resolution near shock fronts than Eulerian computations. However, the former tends to have considerably more complicated implementations when inflow or outflow boundary conditions need to be specified, since they may require element deletion and insertion in some portion of the boundary.

The Lagrangian+remap approach is performed in three separate stages at each time step: The Lagrangian step, the mesh smoothing procedure, and the data remap. Among these, the remap, which can be performed as either a convective partial differential equation solve $[9,10,15]$ or a data transfer process $[16,17]$, has a computational structure quite different from the Lagrangian stage, and aften requires special strategies $\mathrm{n}$ order to preserve conservation.

\footnotetext{
*Corresponding author: Xianyi Zeng. Email: xy.zeng@ duke.edu

Email addresses: xy .zeng@duke.edu (X. Zeng), guglielmo.scovazzi@duke.edu (G. Scovazzi) Preprint submitted to Journal of Computational Physics
} 
In our approach, described in the current work for a single material, an arbitrary Lagrangian-Eulerian (ALE) mesh motion is performed as a preliminary step, followed by a direct (monolithic) solve of the ALE equations of compressible flow dynamics. It is then straightforward to conserve the mass, linear momentum, and the total energy in this direct ALE solve. This solution strategy leads to a monolithic arbitrary Lagrangian-Eulerian (ALE) method. Particularly, in the mesh motion step, we first displace the computational grid by the flow velocity and then apply a chosen mesh smoothing technique to the resulting grid. We consider two different mesh smoothing methods: A Laplacian smoothing and a linear elasticity solve.

The monolithic ALE flow solver is derived following the variational multiscale (VMS) finite element approach, and is inspired by recent developments for pure Lagrangian methods [4, 5, 11]. Hence, we incorporate subgrid-scale modeling into the standard Galerkin formulation of the governing equations to stabilize the system against convection and we use the entropy viscosity proposed by Guermond et al. [18-22] to capture the strong discontinuities. Finally, we employ a second-order explicit predictor/multi-corrector method [11, 23] to integrate the resulting system from one time step to the next.

We extend to ALE simulations the approach recently proposed in [22] to develop entropy-based discontinuity capturing operators for Lagrangian flows. In particular, we designed artificial (mass, viscous, heat) fluxes for this purpose. The specific form of the artificial mass flux proposed here is however different from the regularization discussed in [22, 24], as we cap the entropy viscosity by a von Neumann viscosity proportional to the mesh convective velocity. In the pure Lagrangian case, the mesh convective velocity vanishes and the artificial mass flux is inactive. In this case, our method behaves like most hydrocodes, in that no mass exchange occurs between flow particles (mesh nodes). This property is deemed important for multi-material Lagrangian flows, which will be the object of future work.

Both the variational multiscale stabilization and the artificial viscosity are designed using the discrete residuals of the flow equations. Therefore the proposed method is residual consistent and remains formally second-order accurate for sufficiently smooth solutions. We also would like to point the reader to the recent work of Ricchiuto, Deconinck, Abgrall and coauthors on residual distribution (RD) schemes, which yield stable and accurate dual-volume type nodal unstructured finite element methods for general nonlinear conservation laws [25-27], shallow water equations [28], inviscid flows on arbitrary grids [29], and, more recently, ALE computations [30]. The interested reader can refer to [27] for a comprehensive introduction and survey of recent works. Very recent work of Waltz et al. [31] attacks ALE strong shock computations from the finite volume method perspective.

Monolithic ALE methods have been popularized in fluid-structure interaction (FSI) computations in the context of aeroelasticity [32-36]. There are however some differences in mesh motion strategies between aeroelasticity and shock hydrodynamics, in that the mesh motion for monolithic ALE-FSI is typically determined from the motion of the structure, whereas in the current work the mesh motion is guided by the flow motion. In shock hydrodynamics ALE, one cannot make the assumption any longer that computational grids are nearly equally spaced throughout the computation, whereas this is typically the case when mesh motion is completely guided by the boundary motion in FSI applications.

Another important aspect of the proposed method is that it attempts to address highly transient compressible flows with a stabilized finite element method. To the authors' knowledge, there is effectively no trace in the literature of a similar attempt. For more details on stabilized methods for steady compressible flows, the reader can refer to the survey in [37]. Because we allow more pronounced mesh distortion, it proves crucial to incorporate the Galilean invariance concept in designing the numerical algorithm [38, 39]. Our computational strategy leads to a conservative method, in spite of the fact that we retain primitive variables (density, velocity, and internal energy).

The remainder of the article is organized as follows: The governing equations of the problem are summarized in Section 2, the proposed stabilized variational multiscale method is described in Section 3, and the entropy-based discontinuity capturing operators are presented in Section 4, various choices of mesh motion algorithms are detailed in Section 5, time integration is discussed in Section 6, and results from an extensive battery of numerical tests are shown in Section 7, conclusions are summarized in Section 8 


\section{The governing equations for shock hydrodynamics}

Throughout this article, $\chi$ and $\boldsymbol{x}$ denote the initial and current location of a portion of the computational mesh in a numerical simulation. They are related by a continuous motion map $\varphi: \mathbb{R}^{n_{d}} \times \mathbb{R}^{+} \rightarrow \mathbb{R}^{n_{d}}$ :

$$
\boldsymbol{x}=\varphi(\chi, t),
$$

where $n_{d}$ is the spatial dimension of the problem. We also use the notation $\varphi_{t}(\chi) \stackrel{\text { def }}{=} \varphi(\chi, t)$, so that $\varphi_{0}(\chi)=\varphi(\chi, 0)=\chi$. Analogously, the mesh velocity $\hat{v}$ is defined by:

$$
\hat{\boldsymbol{v}}=\left.\frac{\partial \boldsymbol{x}}{\partial t}\right|_{\chi}=\frac{\partial \varphi}{\partial t} .
$$

For purely Eulerian computations, $\varphi \equiv \chi$ and $\hat{v} \equiv 0$. In what follows, we distinguish between the mesh time-derivative $\left.\frac{\partial \phi}{\partial t}\right|_{\chi}$ (the time derivative at fixed $\chi$ ), and the Eulerian time-derivative, denoted by $\phi_{t}=\left.\frac{\partial \phi}{\partial t}\right|_{x}$.

Shock hydrodynamics is described, in Eulerian coordinates, by the compressible Navier-Stokes equations:

$$
\boldsymbol{U}_{t}+\nabla \cdot\left(\boldsymbol{U} \boldsymbol{v}^{T}+\boldsymbol{G}\right)+\boldsymbol{B}=0,
$$

where

$$
\boldsymbol{U}=\left(\begin{array}{c}
\rho \\
\rho \boldsymbol{v} \\
\rho E
\end{array}\right), \quad \boldsymbol{G}=\left(\begin{array}{c}
0 \\
-\boldsymbol{\sigma} \\
-\boldsymbol{v}^{T} \boldsymbol{\sigma}+\boldsymbol{q}
\end{array}\right), \quad \text { and } \quad \boldsymbol{B}=\left(\begin{array}{c}
0 \\
\boldsymbol{b} \\
\rho(\boldsymbol{b} \cdot \boldsymbol{v}+r)
\end{array}\right) .
$$

Here $\rho, v$, and $E$ are the density, velocity, and total energy per unit mass, respectively, $\nabla$ denotes the gradient operator in $\boldsymbol{x}$, and $\nabla$. is the divergence operator, operating on the second index of $\boldsymbol{U} \boldsymbol{v}^{T}+\boldsymbol{G}$. Also, in the following, the body force $\boldsymbol{b}$, the heat flux $\boldsymbol{q}$, and the heat radiation $r$ are assumed zero for simplicity. The stress tensor is given by

$$
\sigma=-p \boldsymbol{I}+\boldsymbol{\sigma}_{\mathrm{visc}}, \quad \sigma_{\mathrm{visc}}=\rho v \boldsymbol{\epsilon}(\boldsymbol{v}),
$$

where $\boldsymbol{\epsilon}(\boldsymbol{v})=\left(\nabla \boldsymbol{v}+(\nabla \boldsymbol{v})^{T}\right) / 2$ is the symmetric strain rate tensor, $p$ is the thermodynamic pressure, and $v$ is the kinematic viscosity. The total energy density can be expressed as

$$
E=e+\frac{v \cdot v}{2},
$$

where $e$ is the specific internal energy related by the density and pressure by the equation of state

$$
e=e(p, \rho) .
$$

Furthermore, we will consider inviscid flows, for which $v=0$. However the form 2.5 of the equations will still be useful when applying an artificial viscosity technique to numerically capture strong solution discontinuities.

\subsection{Conservative ALE equations for shock hydrodynamics}

In ALE computations, the solution is computed on a moving mesh. Correspondingly, the time derivative holding the grid coordinate fixed is needed, and can be computed from the Eulerian time derivative using

$$
\left.\frac{\partial}{\partial t}\right|_{\chi}=\left.\frac{\partial}{\partial t}\right|_{x}+\hat{\boldsymbol{v}} \cdot \nabla
$$

Then, equation 2.3 becomes

$$
\left.\frac{\partial \boldsymbol{U}}{\partial t}\right|_{\chi}+\boldsymbol{c} \cdot \nabla \boldsymbol{U}+(\nabla \cdot \boldsymbol{v}) \boldsymbol{U}+\nabla \cdot \boldsymbol{G}=0,
$$

where $\boldsymbol{c}=\boldsymbol{v}-\hat{\boldsymbol{v}}$ is the mesh convective velocity. A conservative form of equation 2.97) can be obtained introducing the geometric conservation law:

$$
\left.\frac{\partial \hat{J}}{\partial t}\right|_{\chi}-\hat{J} \nabla \cdot \hat{v}=0
$$


where $\hat{J}=\operatorname{det}\left(\frac{\partial x}{\partial \chi}\right)$ is the Jacobian determinant of the mesh deformation gradient. Combining 2.10 and 2.9 we obtain:

$$
\left.\frac{\partial(\hat{J} \boldsymbol{U})}{\partial t}\right|_{\chi}+\hat{J} \nabla \cdot\left(\boldsymbol{U} \boldsymbol{c}^{T}+\boldsymbol{G}\right)=0 .
$$

We assume $\hat{J}>0$ at all times, which is required for avoiding mesh tangling or inverted elements. For the mesh smoothing strategies described in Section 5, we experience no negative Jacobian determinant in the numerical examples of Section 7

\subsection{The equations for primitive variables}

In order to derive a Galilean invariant stabilization [38-40], when applying the variational multiscale framework, we prefer to construct the stabilization terms using primitive variables. For this purpose, it is instrumental to derive equations for the updates of $v, e$, and $p$. To this end, in 2.9], subtracting from the momentum equation the product of $v$ times the continuity equation yields:

$$
\left.\rho \frac{\partial \boldsymbol{v}}{\partial t}\right|_{\chi}+\rho \boldsymbol{c} \cdot \nabla \boldsymbol{v}-\nabla \cdot \sigma=0
$$

Left-multiplying equation 2.9 with $\left(\left(\frac{1}{2} v \cdot v-e\right),-v^{T}, 1\right)^{T}$ gives the equation for internal energy:

$$
\left.\rho \frac{\partial e}{\partial t}\right|_{\chi}+\rho c \cdot \nabla e-\sigma: \nabla v=0
$$

To derive the equation for the pressure, we replace the partial derivatives in $e$ by partial derivatives in $\rho$ and $p$ using

$$
d e=\left.\frac{\partial e}{\partial \rho}\right|_{p} d \rho+\left.\frac{\partial e}{\partial p}\right|_{\rho} d p
$$

and follow a standard thermodynamics argument [8, 41] for non-dissipative media to obtain:

$$
\left.\frac{\partial p}{\partial t}\right|_{\chi}+\boldsymbol{c} \cdot \nabla p+\rho c_{s}^{2} \nabla \cdot \boldsymbol{v}-\gamma_{G} \boldsymbol{\sigma}_{\mathrm{visc}}: \nabla \boldsymbol{v}=0
$$

Here $c_{s}$ is the speed of sound and $\gamma_{G}=\left(\left.\rho \frac{\partial e}{\partial p}\right|_{\rho}\right)^{-1}$ is the Grüneisen parameter. Note that the parameter $\gamma_{G}$, if no phase transitions occur, can be approximated by a constant, and, in the particular case of a perfect gas, we have $\gamma_{G}=\gamma-1$, where $\gamma$ is the specific heat capacity ratio.

\subsection{Initial and boundary conditions}

Let the initial fluid domain be $\Omega_{0}$, then the initial condition of the problem is given by:

$$
\boldsymbol{U}(\chi, 0)=\boldsymbol{U}_{0}(\chi), \quad \forall \chi \in \Omega_{0}
$$

Defining $\Omega_{t}=\varphi_{t}\left(\Omega_{0}\right)$ and its boundary $\Gamma_{t}=\partial \Omega_{t}$, we assume no topological change of the fluid domain during the computations:

$$
\Gamma_{t}=\varphi_{t}\left(\Gamma_{0}\right) .
$$

Moreover, we suppose that the set of mesh entities on the boundaries also remain unchanged during computations, that is, an internal node remains internal, and a boundary node remains on the boundary.

One of the major motivations in the development of monolithic ALE solvers for shock hydrodynamics is to simplify the treatment of inflow or outflow boundary conditions with respect to Lagrangian+remap multistage algorithms. With this purpose in mind, we consider here three types of boundary conditions, at a velocity boundary $\Gamma_{v}$, a pressure boundary $\Gamma_{p}$, or an Eulerian boundary $\Gamma_{\text {eul }}$, respectively. $\Gamma=\Gamma_{v} \cup \Gamma_{p} \cup \Gamma_{\text {eul }}$ is a partition of the entire boundary $\Gamma$, such that the intersection of any pair of different boundary types has empty interior. We further suppose:

$$
\Gamma_{v, t}=\varphi_{t}\left(\Gamma_{v, 0}\right), \quad \Gamma_{p, t}=\varphi_{t}\left(\Gamma_{p, 0}\right), \quad \Gamma_{\mathrm{eul}, t}=\varphi_{t}\left(\Gamma_{\mathrm{eul}, 0}\right),
$$


and the same property holds for the set of mesh entities on each type of boundaries. On the velocity boundary $\Gamma_{v}$, the boundary condition is given by:

$$
\boldsymbol{v}(\chi, t) \cdot \boldsymbol{n}=v_{n}, \quad \forall \chi \in \Gamma_{\boldsymbol{v}, t},
$$

where $\boldsymbol{n}$ is the unit outer normal vector on $\Gamma_{\boldsymbol{v}, t}$, and $v_{n}$ is the imposed normal component of the flow velocity. For example, in the case of a stationary slip wall, $v_{n}=0$, and in the case of a fluid-structure interface, $v_{n}$ is the normal component of the velocity of the structure. On the pressure boundary $\Gamma_{p}$, the boundary condition is given by:

$$
p(\chi, t)=p_{b}, \quad \forall \chi \in \Gamma_{p, t},
$$

where $p_{b}$ is a specified ambient pressure over the interface $\Gamma_{p, t}$. Because both the velocity boundary condition and the pressure boundary condition are widely used in the context of Lagrangian computations, we assume in addition that on $\Gamma_{v}$ and $\Gamma_{p}$, the mesh velocity is tangential to the boundary curves, i.e.:

$$
(\boldsymbol{v}(\chi, t)-\hat{\boldsymbol{v}}(\chi, t)) \cdot \boldsymbol{n}=0 \quad \text { or } \quad \boldsymbol{c}(\chi, t) \cdot \boldsymbol{n}=0 \quad \forall \chi \in \Gamma_{\boldsymbol{v}, t} \cup \Gamma_{p, t} .
$$

Lastly, we consider an Eulerian boundary $\Gamma_{\text {eul }}$ that does not change in time (hence the name "Eulerian"), so that

$$
\hat{\boldsymbol{v}}(\chi, t) \cdot \boldsymbol{n}=0, \quad \forall \chi \in \Gamma_{\mathrm{eul}, t},
$$

which allow to specify generalized inflow and outflow boundary conditions. The precise treatment of the boundary condition on $\Gamma_{\text {eul }}$ depends on the specific structure of the Riemann problem at the boundary, and is therefore problem dependent. One special case is when the entire solution is imposed at Dirichlet boundaries $\Gamma_{\text {eul-D. Namely, }}$

$$
\boldsymbol{U}=\boldsymbol{U}_{\mathrm{eul}-\mathrm{D}}
$$

Examples of such situation are supersonic inflow boundaries. The remainder of the Eulerian boundary is denoted by $\Gamma_{\text {eul-C }}=\Gamma_{\text {eul }} \backslash \Gamma_{\text {eul-D }}$, where we use the characteristic Riemann invariants decomposition proposed in [42].

\section{The variational multiscale finite element method}

A stabilized finite element method of the equations of transient compressible shock dynamics can be obtained using the variational multiscale analysis [43-45]. For simplicity, but without loss of generality, we consider a semidiscretized form of the equations. The concepts developed here can be generalized to specific time integration strategies, including also space-time algorithms. Since the variational multiscale analysis is based on a local linearization of the equations, it is not sufficient to control numerical spurious oscillations near strong shocks, for which appropriate discontinuity capturing operators are required. For the moment then, we will consider the situation in which flows are smooth and $\boldsymbol{\sigma}=-p \boldsymbol{I}_{n_{d} \times n_{d}}$. We also assume that the mesh velocity $\hat{\boldsymbol{v}}$ is already computed, that is, it is a datum in the problem at hand. Let us define

$$
(f, g)_{\Omega}=\int_{\Omega} f g d \boldsymbol{x}, \quad(\boldsymbol{f}, \boldsymbol{g})_{\Omega}=\int_{\Omega} \boldsymbol{f} \cdot \boldsymbol{g} d \boldsymbol{x}, \quad \text { and } \quad(\boldsymbol{\alpha}, \boldsymbol{\beta})_{\Omega}=\int_{\Omega} \boldsymbol{\alpha}: \boldsymbol{\beta} d \boldsymbol{x}
$$

for two scalars $f$ and $g$, two vectors $\boldsymbol{f}$ and $\boldsymbol{g}$, and two second-order tensors $\boldsymbol{\alpha}$ and $\boldsymbol{\beta}$, respectively. $\Omega$ is any domain and $\boldsymbol{x}$ is its Cartesian coordinate system. Similarly, for any regular boundary portion $\gamma \subset \Gamma$, we define

$$
\langle f, g\rangle_{\gamma}=\int_{\gamma} f g d s, \quad \text { and }\langle\boldsymbol{f}, \boldsymbol{g}\rangle_{\gamma}=\int_{\gamma} \boldsymbol{f} \cdot \boldsymbol{g} d s .
$$

The variational statement of 2.11 consists in finding solutions $\rho, v$, and $e$ in the trial spaces

$$
\begin{aligned}
& \mathcal{S}_{\rho}=\left\{\rho \in H^{1}\left(\Omega_{\chi}\right):\left.\rho\right|_{\Gamma_{\text {eul-D }}}=\rho_{\text {eul }}\right\}, \\
& \mathcal{S}_{\boldsymbol{v}}=\left\{\boldsymbol{v} \in H^{\mathrm{div}}\left(\Omega_{\chi}\right):\left.\boldsymbol{v} \cdot \boldsymbol{n}\right|_{\Gamma_{v}}=v_{n},\left.\boldsymbol{v}\right|_{\Gamma_{\text {eul-D }}}=\boldsymbol{v}_{\text {eul }}\right\}, \\
& \mathcal{S}_{e}=\left\{e \in H^{1}\left(\Omega_{\chi}\right):\left.e\right|_{\Gamma_{\text {eul-D }}}=e_{\text {eul }}\right\},
\end{aligned}
$$


such that

$$
\begin{aligned}
& 0=\frac{d}{d t}(\phi, \rho)_{\Omega_{x}}-(\nabla \phi, \rho \boldsymbol{c})_{\Omega_{x}}+\langle\phi, \rho \boldsymbol{c} \cdot \boldsymbol{n}\rangle_{\Gamma_{\text {eul }} \backslash \Gamma_{\text {eul-D }}}, \\
& 0=\frac{d}{d t}(\boldsymbol{\psi}, \rho \boldsymbol{v})_{\Omega_{x}}-(\nabla \boldsymbol{\psi}, \rho \boldsymbol{v} \otimes \boldsymbol{c}+p \boldsymbol{I})_{\Omega_{x}}+\langle\boldsymbol{\psi}, p \boldsymbol{n}\rangle_{\Gamma \backslash \Gamma_{\text {eul-D }}}+\langle\boldsymbol{\psi}, \rho(\boldsymbol{v} \otimes \boldsymbol{c}) \boldsymbol{n}\rangle_{\Gamma_{\text {eul }} \backslash \Gamma_{\text {eul-D }}}, \\
& 0=\frac{d}{d t}(\phi, \rho E)_{\Omega_{x}}-(\nabla \phi, \rho E \boldsymbol{c}+p \boldsymbol{v})_{\Omega_{x}}+\langle\phi, \rho E \boldsymbol{c} \cdot \boldsymbol{n}\rangle_{\Gamma_{\text {eul }} \backslash \Gamma_{\text {eul-D }}}+\langle\phi, p \boldsymbol{v} \cdot \boldsymbol{n}\rangle_{\Gamma_{\Gamma} \Gamma_{\text {eul-D }}},
\end{aligned}
$$

are satisfied for all test functions $\phi \in \mathcal{V}_{\rho}, \psi \in \mathcal{V}_{\rho v}$, and $\phi \in \mathcal{V}_{\rho E}$, respectively, where

$$
\begin{aligned}
\mathcal{V}_{\rho}=\mathcal{V}_{\rho E} & =\left\{\phi \in H^{1}\left(\Omega_{\chi}\right):\left.\phi\right|_{\Gamma_{\text {eul-D }}}=0\right\}, \quad \text { and } \\
\mathcal{V}_{\rho v} & =\left\{\boldsymbol{\psi} \in H^{\mathrm{div}}\left(\Omega_{\chi}\right):\left.\boldsymbol{\psi} \cdot \boldsymbol{n}\right|_{\Gamma_{\boldsymbol{v}}}=0,\left.\boldsymbol{\psi}\right|_{\Gamma_{\text {eul-D }}}=0\right\} .
\end{aligned}
$$

Trial and test functions move with the mesh coordinate, and their value at fixed $\chi$ remains constant over each time interval. The terms containing time-derivatives in (3.4) have been obtained using the following identity:

$$
\left(\phi,\left.\partial_{t}(\hat{J} \rho)\right|_{\chi}\right)_{\Omega_{\chi}}=\left.\int_{\Omega_{\chi}} \phi \frac{\partial}{\partial t}\right|_{\chi}(\hat{J} \rho) d \chi=\left.\int_{\Omega_{\chi}} \frac{\partial}{\partial t}\right|_{\chi}(\hat{J} \phi \rho) d \chi=\frac{d}{d t} \int_{\Omega_{\chi}} \hat{J} \phi \rho d \chi=\frac{d}{d t} \int_{\Omega_{x}} \phi \rho d \boldsymbol{x}=\frac{d}{d t}(\phi, \rho)_{\Omega_{x}} .
$$

The boundary terms can be derived using integration by parts and the assumptions $2.21,2.22$. For example, in (3.4a), we have

$$
(\phi, \hat{J} \nabla \cdot(\rho \boldsymbol{c}))_{\Omega_{\chi}}=(\phi, \nabla \cdot(\rho \boldsymbol{c}))_{\Omega_{x}}=-(\nabla \phi, \rho \boldsymbol{c})_{\Omega_{x}}+\langle\phi, \rho \boldsymbol{c} \cdot \boldsymbol{n}\rangle_{\Gamma}=-(\nabla \phi, \rho \boldsymbol{c})_{\Omega_{x}}+\langle\phi, \rho \boldsymbol{c} \cdot \boldsymbol{n}\rangle_{\Gamma_{\text {eul }} \backslash \Gamma_{\text {eul-D }}} .
$$

Following a similar strategy, we can also derive the Euler-Lagrangian equations associated with (3.4):

$$
\begin{aligned}
& 0=\left(\phi,\left.\partial_{t}(\hat{J} \rho)\right|_{\chi}\right)_{\Omega_{\chi}}+(\phi, \nabla \cdot(\rho \boldsymbol{c}))_{\Omega_{x}}, \\
& 0=\left(\boldsymbol{\psi},\left.\partial_{t}(\hat{J} \rho \boldsymbol{v})\right|_{\chi}\right) \Omega_{\Omega_{\chi}}+(\boldsymbol{\psi}, \nabla \cdot(\rho \boldsymbol{v} \otimes \boldsymbol{c}+p \boldsymbol{I}))_{\Omega_{x}}, \\
& 0=\left(\phi,\left.\partial_{t}(\hat{J} \rho E)\right|_{\chi}\right)_{\Omega_{\chi}}+(\phi, \nabla \cdot(\rho E \boldsymbol{c}+p \boldsymbol{v}))_{\Omega_{x}} .
\end{aligned}
$$

\subsection{VMS-stabilization I: Coarse-scale equations}

Let $\mathcal{T}=\left\{\Omega_{e}\right\}$ be a tessellation of the domain $\Omega_{\chi}$. The standard Galerkin formulation on the semi-discretized level is obtained by replacing the trial functions and test functions of 3.4 by their numerical approximations. More specifically, the trial spaces are

$$
\begin{aligned}
& \mathcal{S}_{\rho}^{h}=\left\{\rho^{h} \in H^{1}\left(\Omega_{\chi}\right) \cap C\left(\Omega_{\chi}\right):\left.\rho^{h}\right|_{\Omega_{e}} \in P^{1}\left(\Omega_{e}\right), \forall \Omega_{e} \in \mathcal{T} \text { and }\left.\rho^{h}\right|_{\Gamma_{\text {eul-D }}}=\rho_{\text {eul }}\right\}, \\
& \mathcal{S}_{v}^{h}=\left\{\boldsymbol{v}^{h} \in H^{\operatorname{div}}\left(\Omega_{\chi}\right) \cap\left(C\left(\Omega_{\chi}\right)\right)^{n_{d}}:\left.\boldsymbol{v}^{h}\right|_{\Omega_{e}} \in\left(P^{1}\left(\Omega_{e}\right)\right)^{n_{d}}, \forall \Omega_{e} \in \mathcal{T} \text { and }\left.\boldsymbol{v}^{h} \cdot \boldsymbol{n}\right|_{\Gamma_{v}}=v_{n},\left.\boldsymbol{v}^{h}\right|_{\Gamma_{\text {eul-D }}}=\boldsymbol{v}_{\text {eul }}\right\}, \\
& \mathcal{S}_{e}^{h}=\left\{e^{h} \in H^{1}\left(\Omega_{\chi}\right) \cap C\left(\Omega_{\chi}\right):\left.e^{h}\right|_{\Omega_{e}} \in P^{1}\left(\Omega_{e}\right), \forall \Omega_{e} \in \mathcal{T} \text { and }\left.e^{h}\right|_{\Gamma_{\text {eul-D }}}=e_{\text {eul }}\right\},
\end{aligned}
$$

and the corresponding finite-dimensional test function spaces are

$$
\begin{aligned}
\mathcal{V}_{\rho}^{h}=\mathcal{V}_{\rho E}^{h} & =\left\{\phi^{h} \in H^{1}\left(\Omega_{\chi}\right) \cap C\left(\Omega_{\chi}\right):\left.\phi^{h}\right|_{\Omega_{e}} \in P^{1}\left(\Omega_{e}\right), \forall \Omega_{e} \in \mathcal{T} \text { and }\left.\phi^{h}\right|_{\Gamma_{\text {eul-D }}}=0\right\}, \\
\mathcal{V}_{\rho \boldsymbol{v}}^{h} & =\left\{\boldsymbol{\psi}^{h} \in H^{\mathrm{div}}\left(\Omega_{\chi}\right) \cap\left(C\left(\Omega_{\chi}\right)\right)^{n_{d}}:\left.\boldsymbol{\psi}^{h}\right|_{\Omega_{e}} \in\left(P^{1}\left(\Omega_{e}\right)^{n_{d}}, \forall \Omega_{e} \in \mathcal{T} \text { and }\left.\boldsymbol{\psi}^{h} \cdot \boldsymbol{n}\right|_{\Gamma_{v}}=0,\left.\boldsymbol{\psi}^{h}\right|_{\Gamma_{\text {eul-D }}}=0\right\} .\right.
\end{aligned}
$$

We extend the VMS approach for Lagrangian shock hydrodynamics in [11] to the monolithic ALE framework. More specifically, the function spaces above are written as the direct sum of finite-dimensional, coarse-scale subspaces and infinite-dimensional, fine-scale subspaces (subgrid-scale subspaces):

$$
\mathcal{S}_{\rho}=\mathcal{S}_{\rho}^{h} \oplus \mathcal{S}_{\rho}^{\prime}, \quad \mathcal{S}_{\boldsymbol{v}}=\mathcal{S}_{v}^{h} \oplus \mathcal{S}_{v}^{\prime}, \quad \mathcal{S}_{e}=\mathcal{S}_{e}^{h} \oplus \mathcal{S}_{e}^{\prime},
$$

and

$$
\mathcal{V}_{\rho}=\mathcal{V}_{\rho}^{h} \oplus \mathcal{V}_{\rho}^{\prime}, \quad \mathcal{V}_{\rho v}=\mathcal{V}_{\rho v}^{h} \oplus \mathcal{V}_{\rho v}^{\prime}, \quad \mathcal{V}_{\rho E}=\mathcal{V}_{\rho E}^{h} \oplus \mathcal{V}_{\rho E}^{\prime}
$$


Here we define the direct non-orthogonal sums in 3.10 and 3.11 using the $H^{1}$-projector for scalar fields and the $H^{\text {div }}$-projector for vector fields. This approach allows simplifications in the treatment of the inter-element boundary terms in the stabilized formulation, as explained in [11]. Correspondingly, we write:

$$
\rho=\rho^{h}+\rho^{\prime}, \quad \boldsymbol{v}=\boldsymbol{v}^{h}+\boldsymbol{v}^{\prime}, \quad e=e^{h}+e^{\prime}, \quad p=p^{h}+p^{\prime},
$$

and, similarly, for the test functions, $\phi=\phi^{h}+\phi^{\prime}$ and $\boldsymbol{\psi}=\boldsymbol{\psi}^{h}+\boldsymbol{\psi}^{\prime}$. Applying the decomposition 3.12 to the EulerLagrangian equations 3.7 , tested onto the coarse-scale test functions $\left(\phi^{h}, \psi^{h}, \phi^{h}\right)$ yields:

$$
\begin{aligned}
& 0=\left(\phi^{h},\left.\partial_{t}\left(\hat{J}\left(\rho^{h}+\rho^{\prime}\right)\right)\right|_{\chi}\right)_{\Omega_{\chi}}+\left(\phi^{h}, \nabla \cdot\left(\left(\rho^{h}+\rho^{\prime}\right) \boldsymbol{c}^{h}\right)\right)_{\Omega_{x}}, \\
& 0=\left(\boldsymbol{\psi}^{h},\left.\partial_{t}\left(\hat{J}\left(\rho^{h}+\rho^{\prime}\right)\left(\boldsymbol{v}^{h}+\boldsymbol{v}^{\prime}\right)\right)\right|_{\chi}\right)_{\Omega_{\chi}}+\left(\boldsymbol{\psi}^{h}, \nabla \cdot\left(\left(\rho^{h}+\rho^{\prime}\right)\left(\boldsymbol{v}^{h}+\boldsymbol{v}^{\prime}\right) \otimes \boldsymbol{c}^{h}+\left(p^{h}+p^{\prime}\right) \boldsymbol{I}\right)\right)_{\Omega_{x},}, \\
& 0=\left(\phi^{h},\left.\partial_{t}\left(\hat{J}\left(\rho^{h}+\rho^{\prime}\right)\left(E^{h}+E^{\prime}\right)\right)\right|_{\chi}\right)_{\Omega_{\chi}}+\left(\phi^{h}, \nabla \cdot\left(\left(\rho^{h}+\rho^{\prime}\right)\left(E^{h}+E^{\prime}\right) \boldsymbol{c}^{h}+\left(p^{h}+p^{\prime}\right)\left(\boldsymbol{v}^{h}+\boldsymbol{v}^{\prime}\right)\right)\right)_{\Omega_{x}} .
\end{aligned}
$$

Let us now consider the following simplifying assumptions:

(a) There is no subgrid-scale mesh advection velocity, i.e., $\boldsymbol{c}^{\prime} \equiv 0$. This choice is motivated by the fact that, in the Lagrangian limit, the mesh convective velocity should vanish identically.

(b) Time-derivatives of the subgrid-scale quantities are neglected, using a quasi-static approach.

(c) Subgrid-scale quantities are assumed small, so that products of fine-scale terms can be neglected. This assumption is effectively implying linearization of the fine-scale terms in the equations.

(d) Subgrid-scale quantities vanish on the outer boundary $\Gamma$.

Hence, we obtain that 3.13 simplifies into

$$
\begin{aligned}
& 0=\left(\phi^{h},\left.\partial_{t}\left(\hat{J} \rho^{h}\right)\right|_{\chi}\right)_{\Omega_{\chi}}+\left(\phi^{h}, \nabla \cdot\left(\left(\rho^{h}+\rho^{\prime}\right) \boldsymbol{c}^{h}\right)\right)_{\Omega_{x}}, \\
& 0=\left(\boldsymbol{\psi}^{h},\left.\partial_{t}\left(\hat{J} \rho^{h} \boldsymbol{v}^{h}\right)\right|_{\chi}\right)_{\Omega_{\chi}}+\left(\boldsymbol{\psi}^{h}, \nabla \cdot\left(\left(\rho^{h} \boldsymbol{v}^{h}+\rho^{\prime} \boldsymbol{v}^{h}+\rho^{h} \boldsymbol{v}^{\prime}\right) \otimes \boldsymbol{c}^{h}+\left(p^{h}+p^{\prime}\right) \boldsymbol{I}\right)\right)_{\Omega_{x}}, \\
& 0=\left(\phi^{h},\left.\partial_{t}\left(\hat{J} \rho^{h} E^{h}\right)\right|_{\chi}\right)_{\Omega_{\chi}}+\left(\phi^{h}, \nabla \cdot\left(\left(\rho^{h} E^{h}+\rho^{\prime} E^{h}+\rho^{h} E^{\prime}\right) \boldsymbol{c}^{h}+\left(p^{h} \boldsymbol{v}^{h}+p^{\prime} \boldsymbol{v}^{h}+p^{h} \boldsymbol{v}^{\prime}\right)\right)\right)_{\Omega_{x}} .
\end{aligned}
$$

and, integrating by parts in space on every element of the discretization,

$$
\begin{aligned}
0= & \frac{d}{d t}\left(\phi^{h}, \rho^{h}\right)_{\Omega_{x}}-\left(\nabla \phi^{h},\left(\rho^{h}+\rho^{\prime}\right) \boldsymbol{c}^{h}\right)_{\Omega_{x}}+\left\langle\phi^{h}, \rho^{h} \boldsymbol{c}^{h} \cdot \boldsymbol{n}\right\rangle_{\Gamma_{\text {eul-C }}}, \\
0= & \frac{d}{d t}\left(\boldsymbol{\psi}^{h}, \rho^{h} \boldsymbol{v}^{h}\right)_{\Omega_{x}}-\left(\nabla \boldsymbol{\psi}^{h},\left(\rho^{h} \boldsymbol{v}^{h}+\rho^{\prime} \boldsymbol{v}^{h}+\rho^{h} \boldsymbol{v}^{\prime}\right) \otimes \boldsymbol{c}^{h}+\left(p^{h}+p^{\prime}\right) \boldsymbol{I}\right)_{\Omega_{x}}+ \\
& \left\langle\boldsymbol{\psi}^{h}, p^{h} \boldsymbol{n}\right\rangle_{\Gamma \backslash \Gamma_{\text {eul-D }}}+\left\langle\boldsymbol{\psi}^{h}, \rho^{h}\left(\boldsymbol{v}^{h} \otimes \boldsymbol{c}^{h}\right) \boldsymbol{n}\right\rangle_{\Gamma \backslash \Gamma_{\text {eul-D }},} \\
0= & \frac{d}{d t}\left(\phi^{h}, \rho^{h} E^{h}\right)_{\Omega_{x}}-\left(\nabla \phi^{h},\left(\rho^{h} E^{h}+\rho^{\prime} E^{h}+\rho^{h} E^{\prime}\right) \boldsymbol{c}^{h}+\left(p^{h} \boldsymbol{v}^{h}+p^{\prime} \boldsymbol{v}^{h}+p^{h} \boldsymbol{v}^{\prime}\right)\right)_{\Omega_{x}}+\left\langle\phi^{h}, \rho^{h} E^{h} \boldsymbol{c}^{h}\right\rangle_{\Gamma_{\text {eul-C}}} .
\end{aligned}
$$

To obtain 3.15), we use the properties of the fine-scale projectors. Considering as an example the continuity equation 3.14a, and applying element-by-element integration by parts, we obtain:

$$
\left(\phi^{h}, \nabla \cdot\left(\left(\rho^{h}+\rho^{\prime}\right) \boldsymbol{c}^{h}\right)\right)_{\Omega_{x}}=-\left(\nabla \phi^{h},\left(\rho^{h}+\rho^{\prime}\right) \boldsymbol{c}^{h}\right)_{\Omega_{x}}+\left\langle\phi^{h},\left(\rho^{h}+\rho^{\prime}\right) \boldsymbol{c}^{h} \cdot \boldsymbol{n}\right\rangle_{\Gamma_{\text {eul- } \mathrm{C}}}+\sum_{\gamma \in \Gamma^{\prime}}\left\langle\phi^{h}, \llbracket\left(\rho^{h}+\rho^{\prime}\right) \boldsymbol{c}^{h} \rrbracket\right\rangle_{\gamma},
$$

where $\Gamma^{\prime}$ is the collection of inner element boundaries, and $\llbracket \boldsymbol{f} \rrbracket=\boldsymbol{f}^{+} \boldsymbol{n}^{+}+\boldsymbol{f}^{-} \boldsymbol{n}^{-}$denotes the usual jump operator across an internal element boundary. Due to assumption (d), $\rho^{\prime}$ vanishes on $\Gamma_{\text {eul-C }}$, hence $\left\langle\phi^{h}, \rho^{\prime} \boldsymbol{c}^{h} \cdot \boldsymbol{n}\right\rangle_{\Gamma_{\text {eul-C }}}=0$. Furthermore, we define the subgrid-scale projector operator for density as $\mathbb{P}_{\rho}^{\prime}: H^{1}(\Omega) \rightarrow H_{0}^{1}(\Omega)$, that is, $\rho^{\prime} \in \mathcal{S}_{\rho}^{\prime} \subset H_{0}^{1}(\Omega)$, the space of functions in $H^{1}(\Omega)$ with zero trace. Following Prop. III.1.1 of Brezzi and Fotin [46], this condition is equivalent to enforcing that $\sum_{\gamma \in \Gamma^{\prime}}\left\langle\phi^{h}, \llbracket \rho^{\prime} \boldsymbol{c}^{h} \rrbracket\right\rangle_{\gamma}$ vanishes for every $\phi^{h}$. The remaining term $\sum_{\gamma \in \Gamma^{\prime}}\left\langle\phi^{h}, \llbracket \rho^{h} \boldsymbol{c}^{h} \rrbracket\right\rangle_{\gamma}$ is clearly zero because both $\rho^{h}$ and $c^{h}$ are globally continuous. Hence, 3.16 reduces to 3.15a, and a similar procedure can be used to derive 3.15b and 3.15c. 


\subsection{VMS-stabilization II: Fine-scale equations}

In order to estimate the fine-scale part of the solution, the governing equations are tested against fine-scale test functions. Using again the continuity equation as an example, we test $3.7 \mathrm{a}$ ) against $\phi^{\prime} \in \mathcal{V}_{\rho}^{\prime}$ and obtain

$$
0=\left(\phi^{\prime},\left.\partial_{t}\left(\hat{J}\left(\rho^{h}+\rho^{\prime}\right)\right)\right|_{\chi}\right)_{\Omega_{\chi}}+\left(\phi^{\prime}, \nabla \cdot\left(\left(\rho^{h}+\rho^{\prime}\right) c^{h}\right)\right)_{\Omega_{x}}
$$

and, segregating coarse-scale quantities to the right hand side,

$$
\left(\phi^{\prime},\left.\partial_{t}\left(\hat{J} \rho^{\prime}\right)\right|_{\chi}\right)_{\Omega_{\chi}}+\left(\phi^{\prime}, \nabla \cdot\left(\rho^{\prime} \boldsymbol{c}^{h}\right)\right)_{\Omega_{x}}=-\left(\phi^{\prime},\left.\partial_{t}\left(\hat{J} \rho^{h}\right)\right|_{\chi}+\hat{J} \nabla \cdot\left(\rho^{h} c^{h}\right)\right)_{\Omega_{\chi}}=\left(\phi^{\prime},-\operatorname{Res}_{\rho}^{h}\right)_{\Omega_{x}},
$$

where the coarse-scale residual for density is defined as

$$
\operatorname{Res}_{\rho}^{h}=\left.\frac{\partial \rho^{h}}{\partial t}\right|_{\chi}+c^{h} \cdot \nabla \rho^{h}+\rho^{h} \nabla \cdot v^{h}
$$

The structure of equation 3.18 motivates the following subgrid-scale density approximation:

$$
\rho^{\prime}=-\tau \operatorname{Res}_{\rho}^{h}
$$

where $\tau$ is a stabilization parameter with the dimensions of an intrinsic time scale of the problem under consideration. This approximation could be better understood if we interpret 3.18 as finding the weak solution $\rho^{\prime} \in \mathcal{S}_{\rho}^{\prime}$ associated with the problem:

$$
\left.\frac{\partial\left(\hat{J} \rho^{\prime}\right)}{\partial t}\right|_{\chi}+\hat{J} \nabla \cdot\left(c^{h} \rho^{\prime}\right)=-\hat{J} \operatorname{Res}_{\rho}^{h} \quad \text { or }\left.\quad \frac{\partial \rho^{\prime}}{\partial t}\right|_{\chi}+c^{h} \cdot \nabla \rho^{h}+\rho^{h} \nabla \cdot v^{h}=-\operatorname{Res}_{\rho}^{h},
$$

where the test functions are selected in $\mathcal{V}_{\rho}^{\prime}$. This differential equation motivates a linear relationship between the coarse-scale residual and the corresponding fine-scale variable. In accordance to VMS approach [27, 43, 47], $\tau$ can be considered as the average action of the Green's function operator over an element. The stabilizing effects of fine-scale quantities 3.20 could also be analyzed from the point of view of the space-time VMS framework for hyperbolic systems (see [48] for an example in the context of the wave equation). Similarly, we define the coarse-scale residuals for velocity, internal energy, and pressure, respectively, as

$$
\begin{aligned}
& \operatorname{Res}_{v}^{h}=\left.\frac{\partial \boldsymbol{v}^{h}}{\partial t}\right|_{\chi}+c^{h} \cdot \nabla \boldsymbol{v}^{h}+\frac{1}{\rho^{h}} \nabla p^{h}, \\
& \operatorname{Res}_{e}^{h}=\left.\frac{\partial e^{h}}{\partial t}\right|_{\chi}+c^{h} \cdot \nabla e^{h}+\frac{p^{h}}{\rho^{h}} \nabla \cdot v^{h}, \\
& \operatorname{Res}_{p}^{h}=\left.\frac{\partial p^{h}}{\partial t}\right|_{\chi}+c^{h} \cdot \nabla p^{h}+\rho^{h} c_{s}^{h 2} \nabla \cdot v^{h},
\end{aligned}
$$

and approximate $\boldsymbol{v}^{\prime}, e^{\prime}$, and $p^{\prime}$ by

$$
\boldsymbol{v}^{\prime}=-\tau \operatorname{Res}_{\boldsymbol{v}}^{h}, \quad e^{\prime}=-\tau \operatorname{Res}_{e}^{h}, \quad \text { and } \quad p^{\prime}=-\tau \operatorname{Res}_{p}^{h} .
$$

The discrete residuals are evaluated by interpolating the solution and computing its gradients at quadrature points. For example, for a tetrahedral grid, Res $\boldsymbol{v}_{\boldsymbol{v}}^{h}$ is evaluated by interpolating the linear fields $\boldsymbol{v}^{h}, \boldsymbol{c}^{h}$, and $\rho^{h}$ at the quadrature point, and combining them with the (elment-wise constant) gradients $\nabla \boldsymbol{v}^{h}$ and $\nabla p^{h}$. Lastly, we linearize (2.6) and approximate $E^{\prime}$ (used in $\left.3.15 \mathrm{c}\right)$ ) as

$$
E^{\prime}=e^{\prime}+v^{h} \cdot v^{\prime}
$$

The parameter $\tau$ is related to the average of the fine-scale Green's function over an element of the discretization, and can be estimated as in [39, 49]. In this work, we adopt the strategy of defining the stabilization matrix for the prime variables as a multiple of the identity matrix, so that only one scalar $\tau$ is required to define all subgrid-scale 
variables. Further modifications to the stabilization matrix may be required for low-Mach flow computations, as suggested in [50], although this topic is outside the scope of the current work. In the present work, $\tau$ is given by

$$
\tau=\frac{c_{\tau} c_{\text {geom }} \Delta t}{2 \sqrt{1+\left(\alpha_{E}^{h}\right)^{2}}}, \quad \text { with } \quad \alpha_{E}^{h}=\frac{\sup _{x \in \Omega_{e}}\left(c_{s}^{h}+\left\|c^{h}\right\|\right) \Delta t}{h_{e}} .
$$

Here $h_{e}$ is the size of the element that is defined by:

$$
h_{e}=c_{\mathrm{geom}}\left|\Omega_{e}\right|^{\frac{1}{n_{d}}}
$$

where $\left|\Omega_{e}\right|$ is the measure of the element $\Omega_{e} . \quad\left\|c^{h}\right\|$ is the local mesh convective speed, $\Delta t$ is the time-step size, $\alpha_{E}^{h}$ is the local Courant number, and $c_{\tau}$ and $c_{\text {geom }}$ are two constants. In $3.27 p_{2}$, the supremum sup $\operatorname{se}_{x \in \Omega_{e}}$ is computed as the maximum of $c_{s}^{h}+\left\|\boldsymbol{c}^{h}\right\|$ at each node of the element. The range $c_{\tau} \in[2,5]$ is typically used. In this work, we find that $c_{\tau}=2.5$ works well for all the numerical problems considered. $c_{\text {geom }}$ accounts for the topology of the element considered, and takes the values $c_{\text {geom }}=\sqrt{4 / \sqrt{3}} \approx 1.5$ for triangles and $c_{\text {geom }}=\sqrt[3]{12 / \sqrt{2}} \approx 2.0$ for tetrahedrons.

The choice of the element size $h_{e}$ may influence the numerical results [12, 22]. The particular choice (3.28) stems from the authors' experience with Lagrangian shock hydrodynamics. The reason for the use of a Jacobian (volume) based element length is to avoid large discrepancies in size between neighboring elements, especially in proximity of shock fronts in Lagrangian or ALE computations. A full investigation of all possible choices of computing $h_{e}$ is beyond the scope of this work; we, however, present a simple comparison of three different strategies in Section 7.3.1 for a Lagrangian computation of Sedov blast wave on an anisotropic grid.

\section{The entropy artificial viscosity}

Because the VMS approach is based on a local linearization/perturbation assumption, it is not suitable to capture strong shocks in nonlinear computations. To obviate this issue, we introduce an entropy-based artificial viscosity inspired by the work in [18-22] on discontinuity capturing operators. The key idea is that the entropy $s$ of the flow remains constant along fluid particle paths if solutions are smooth, whereas it jumps across strong discontinuities (such as shocks). It is therefore possible to use the residual of the entropy equation to detect the location of shocks and scale the artificial viscosity. We describe the approach in the specific case of ideal gases, although this methodology is very general and widely applicable to other classes of fluids. For an ideal gas, we define the entropy as (see Guermond et al. [22]):

$$
s=\frac{(\gamma+1) \rho}{\gamma-1}\left(\frac{p}{\rho^{\gamma}}\right)^{\frac{1}{\gamma+1}}=\frac{\gamma+1}{\gamma-1}(p \rho)^{\frac{1}{\gamma+1}},
$$

where $\gamma$ is the specific heat capacity ratio. The corresponding entropy flux is $f=s \boldsymbol{v}$, where $\boldsymbol{v}$ is the flow velocity, such that the following inequality holds:

$$
s_{t}+\nabla \cdot \boldsymbol{f} \geqslant 0, \quad \text { or }\left.\quad \frac{\partial s}{\partial t}\right|_{\chi}+\boldsymbol{c} \cdot \nabla s+s \nabla \cdot \boldsymbol{v} \geqslant 0 .
$$

We can then define an entropy residual

$$
\operatorname{Res}_{s}^{h}=\left.\frac{\partial s^{h}}{\partial t}\right|_{\chi}+c^{h} \cdot \nabla s^{h}+s^{h} \nabla \cdot v^{h} \geqslant 0,
$$

expected to be small ( $\sim O\left(h^{2}\right)$ for a second-order method) when the solution is smooth, and to be finite when there is a shock. The artificial entropy-based kinematic viscosity $v_{\text {ent }}$ is thus computed as

$$
v_{\mathrm{ent}}=S\left(c_{\mathrm{ent}} \tilde{h}_{e}^{2} \frac{\sup _{\Omega_{e}}\left|\operatorname{Res}_{s}^{h}\right|}{|| s-\bar{s}||_{\infty}+\epsilon \bar{s}}\right), \quad \bar{s}=\frac{1}{\left|\Omega_{x}\right|} \int_{\Omega_{x}} s d \boldsymbol{x},
$$




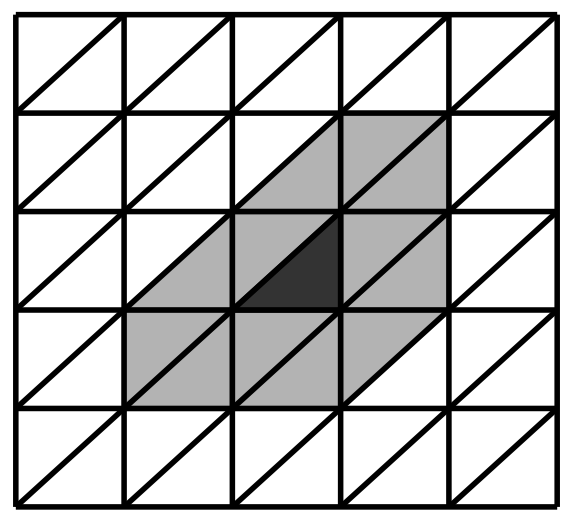

Figure 4.1: Computing the smoothed artificial viscosity: smoothed viscosity of the dark element is the maximum of the non-smoothed viscosity of all the shaded elements.

where $S(\cdot)$ is an appropriate smoothing operator and $c_{\text {ent }}$ a control parameter. Because $\operatorname{Res}_{s}^{h}$ is evaluated at each quadrature point, we take the supremum over each element $\Omega_{e}$ to obtain a representative entropy residual. The denominator $\|s-\bar{s}\|_{\infty}+\epsilon \bar{S}$ is a measure of current entropy scale; and $\epsilon=1 e-10$ is a small number to avoid division by zero. The modified length scale $\tilde{h}_{e}$ is computed as:

$$
\tilde{h}_{e}=h_{e} \min \left(1,\left|\Omega_{e ; 0}\right|^{\frac{1}{n_{d}}} /\left|\Omega_{e}\right|^{\frac{1}{n_{d}}}\right),
$$

where $h_{e}$ is given by $3.28 ;\left|\Omega_{e ; 0}\right|$ and $\left|\Omega_{e}\right|$ denote the measures of the element in the initial and current configurations, respectively. The motivation of taking the minimum in 4.5 is to avoid too large entropy viscosity for an element undergoing large expansion during ALE or Lagrangian computations. In this scenario, 4.5 ensures that the entropy viscosity scales with the square of the initial element length, which represents the user's expectation of the grid resolution.

Because the definition of entropy is in general not unique, the typical value of $c_{\text {ent }}$ depends on the specific definition of the entropy that is used to compute the viscosity. For the particular entropy 4.1, we find the value $c_{\text {ent }}=40.0$ works well for a large collection of shock dynamics problems.

For a strong shock it often happens that the entropy residual is large only in a single layer of elements at the shock front. If no smoother is applied, then the viscosity $v_{\mathrm{ent}}^{e}=c_{\mathrm{ent}} \tilde{h}_{e}^{2} \sup _{\Omega_{e}}\left|\operatorname{Res}_{s}^{h}\right| /\left(\| s-\left.\bar{s}\right|_{\infty}+\epsilon \bar{s}\right)$ is only active in a layer of very few (1-2) elements. This feature is not adequate, for example, in regions where multiple shocks interact causing wide oscillations in the viscosity. A simple smoother $S(\cdot)$ can then be introduced, by defining the smoothed viscosity over an element $\Omega_{e}$ as the maximum of all the non-smoothed viscosities of elements topologically connected to $\Omega_{e}$, that is:

$$
\left.v_{\mathrm{ent}}\right|_{\Omega_{e}}=\max _{\mathcal{T}_{e^{\prime}}: \overline{\Omega_{e}} \cap \overline{\mathcal{T}_{e^{\prime}}} \neq \varnothing} v_{\mathrm{ent}}^{e^{\prime}},
$$

where $v_{\text {ent }}^{e^{\prime}}$ is the non-smoothed viscosity over the element $\mathcal{T}_{e^{\prime}}$. This procedure is illustrated in Figure 4.1. where the smoothed viscosity of the element in dark $\left(\Omega_{e}\right)$ is the maximum of the non-smoothed viscosities of all the shaded elements (including $\Omega_{e}$ itself).

The residual-based viscosity $v_{\text {ent }}$ has the advantage that when the shock is weak, smooth solutions can be achieved with only a small amount of additional dissipation. Instead, in the case of strong shocks, entropy production may result excessive for the purpose of stabilization. A robust way to implement the entropy viscosity is to cap it with the value of the von Neumann and Richtmyer [3, 51] viscosities:

$$
\varepsilon_{\max }=c_{\max } h_{e}\left\|\boldsymbol{c}^{h}\right\|, \quad v_{\max }=c_{\max } h_{e}\left(c_{s}^{h}+\left\|\boldsymbol{c}^{h}\right\|\right),
$$

where the $c_{\max }=0.5$. The two values $\varepsilon_{\max }$ and $v_{\max }$ are used as the upper bounds of the artificial viscosities in the artificial mass flux and the artificial viscous stress, respectively. The motivation for using $\left\|c^{h}\right\|$ in $[4.7\}_{1}$ is that the mass is convected by the mesh convective velocity $\boldsymbol{c}^{h}$. 
The actual artificial viscosities $\varepsilon_{\text {art }}$ and $v_{\text {art }}$ are defined as

$$
\varepsilon_{\mathrm{art}}=\min \left(\varepsilon_{\mathrm{max}}, v_{\mathrm{ent}}\right), \quad v_{\mathrm{art}}=\min \left(v_{\max }, v_{\mathrm{ent}}\right) .
$$

These formula is dimensionally consistent, since the normalization of the global entropy scale is used in (4.4). Once 4.8 is computed, the terms $\left(\nabla \phi^{h}, \varepsilon_{\text {art }} \nabla \rho^{h}\right)_{\Omega_{x}},\left(\nabla \psi^{h}, \rho^{h} v_{\text {art }} \boldsymbol{\epsilon}\left(\boldsymbol{v}^{h}\right)\right)_{\Omega_{x}}$, and $\left(\nabla \phi^{h}, \rho^{h} v_{\text {art }} \boldsymbol{\epsilon}\left(\boldsymbol{v}^{h}\right) \boldsymbol{v}^{h}+\kappa_{\text {art }} \nabla T^{h}\right)_{\Omega_{x}}$ are added to the right-hand sides of the variational equations (3.4a), 3.4b), and (3.4c), respectively, to obtain the final weak form of the proposed method:

$$
\begin{aligned}
0= & \frac{d}{d t}\left(\phi^{h}, \rho^{h}\right)_{\Omega_{x}}-\left(\nabla \phi^{h},\left(\rho^{h}+\rho^{\prime}\right) \boldsymbol{c}^{h}-\varepsilon_{\mathrm{art}} \nabla \rho^{h}\right)_{\Omega_{x}}+\left\langle\phi^{h}, \rho^{h} \boldsymbol{c}^{h} \cdot \boldsymbol{n}\right\rangle_{\Gamma_{\text {eul-C }}}, \\
0= & \frac{d}{d t}\left(\boldsymbol{\psi}^{h}, \rho^{h} \boldsymbol{v}^{h}\right)_{\Omega_{x}}-\left(\nabla \boldsymbol{\psi}^{h},\left(\rho^{h} \boldsymbol{v}^{h}+\rho^{h} \boldsymbol{v}^{\prime}+\rho^{\prime} \boldsymbol{v}^{h}\right) \otimes \boldsymbol{c}^{h}+\left(p^{h}+p^{\prime}\right) \boldsymbol{I}-\rho^{h} v_{\mathrm{art}} \boldsymbol{\epsilon}\left(\boldsymbol{v}^{h}\right)\right)_{\Omega_{x}}+ \\
& \left\langle\boldsymbol{\psi}^{h}, p^{h} \boldsymbol{n}\right\rangle_{\Gamma \backslash \Gamma_{\text {eul-D }}}+\left\langle\boldsymbol{\psi}^{h}, \rho^{h}\left(\boldsymbol{v}^{h} \otimes \boldsymbol{c}^{h}\right) \boldsymbol{n}\right\rangle_{\Gamma_{\text {eul-C }}}, \\
0= & \frac{d}{d t}\left(\phi^{h}, \rho^{h} E^{h}\right)_{\Omega_{x}}-\left(\nabla \phi^{h},\left(\left(\rho^{h} E^{h}+\rho^{\prime} E^{h}+\rho^{h} E^{\prime}\right) \boldsymbol{c}^{h}+\left(p^{h} \boldsymbol{v}^{h}+p^{h} \boldsymbol{v}^{\prime}+p^{\prime} \boldsymbol{v}^{h}\right)-\rho^{h} v_{\mathrm{art}} \boldsymbol{\epsilon}\left(\boldsymbol{v}^{h}\right)-\kappa_{\mathrm{art}} \nabla T^{h}\right)_{\Omega_{x}}+\right. \\
& \left\langle\phi^{h}, \rho^{h} E^{h} \boldsymbol{c}^{h} \cdot \boldsymbol{n}\right\rangle_{\Gamma_{\text {eul-C }}}+\left\langle\phi^{h}, p^{h} \boldsymbol{v}^{h} \cdot \boldsymbol{n}\right\rangle_{\Gamma \backslash \Gamma_{\text {eul-D }}} .
\end{aligned}
$$

Here

$$
\kappa_{\mathrm{art}}=\frac{\rho^{h} v_{\mathrm{art}}}{\gamma-1},
$$

is an artificial diffusivity, and $T^{h}$ is the temperature, given by

$$
T^{h}=(\gamma-1) e^{h},
$$

for ideal gases and the subgrid-scale variables in (4.9) are computed using (3.20, , 3.25) and 3.26).

Remark: The final semi-discretized form 4.9) is both globally conservative for mass, linear momentum, and total energy. A global conservation statement can be derived by taking $\phi^{h} \equiv 1$ and $\psi^{h}=\boldsymbol{e}_{i}, i=1, \cdots, n_{d}$, the unit vectors in $\mathbb{R}^{n_{d}}$, when $\Gamma_{\text {eul-D }}=\varnothing$ and $\Gamma_{v}=\varnothing$. For more details see [52, 53].

Remark: The proposed method is locally mass conservative in the Lagrangian limit regardless of the value of $\varepsilon_{\text {art }}$. In fact, the derivations in [52, 53] can be adapted to construct nodal mass fluxes and establish a mass balance statement at each node. In the Lagrangian limit, note in particular that $\left\|c^{h}\right\|$ vanishes in 4.7 , and $\varepsilon_{\max }=0$. Consequently, the artificial mass flux vanishes, an important feature in Lagrangian computations. The proposed method is also locally conservative for the momentum and energy equations, following arguments similar to the ones presented in [52, 53].

\section{The mesh motion algorithm}

We describe a few options to move the mesh from its location $\boldsymbol{x}_{n}$ at $t_{n}$ to $\boldsymbol{x}_{n+1}$ at $t_{n+1}$. Mesh motion techniques may be tailored to specific problems and of course vary in computational cost. The exploration of the various options in this direction is outside the scope of this article, and we focus only on a few general strategies.

\subsection{The Laplacian smoothing algorithm}

A more versatile mesh updating algorithm is to use the Laplacian smoothing operator [54, 55]. Particularly, it works well with all the boundary conditions described in Section 2.3 Our algorithm is composed of three steps. First, we perform a Lagrangian update of the mesh positions by using

$$
\boldsymbol{x}^{\mathrm{lag}}=\boldsymbol{x}_{n}+\frac{1}{2} \Delta t\left(\boldsymbol{v}_{n}+\boldsymbol{v}_{n+1}\right)
$$

Since the new mesh position may violate the boundary requirements 2.21 and 2.22 , we correct the mesh point location on the Eulerian boundaries as follows:

$$
\boldsymbol{x}^{\mathrm{corr}}=\left\{\begin{array}{ll}
\boldsymbol{x}_{n} & \text { if } \boldsymbol{x}_{n} \in \Gamma_{\mathrm{eul}, t_{n}}, \\
\boldsymbol{x}^{\text {lag }} & \text { otherwise }
\end{array} .\right.
$$


Note that the pull-back of boundary nodes to their original configuration at $t_{n}$ may squeeze the elements near the Eulerian boundaries. However, when we compute the time step size by using the Courant number less than 1.0 with a conservative element length estimate, this procedure will not in general cause mesh tangling.

In the third stage, we apply the Laplacian smoothing operator to the mesh displacement field $\boldsymbol{d}=\boldsymbol{x}^{\text {corr }}-\boldsymbol{x}_{n}$. We describe the operator for nodal elements: Suppose $\mathcal{T}=\left\{\Omega_{e}\right\}$ is a tessellation of the domain $\Omega_{\chi}$ with $N_{\text {node }}$ nodes. We denote by $\boldsymbol{d}_{A}, A=1, \cdots, N_{\text {node }}$ the nodal mesh displacement vector associated with the node with mesh coordinate $\chi_{A}$. Let $N_{A}$ be the shape function corresponding to the mesh position $\Omega_{n}$, the consistent and lumped mass matrices are given by:

$$
\boldsymbol{M}=\left[M_{A B}\right], \quad M_{A B}=\int_{\Omega_{x_{n}}} N_{A} N_{B} d \boldsymbol{x}_{n},
$$

and

$$
\boldsymbol{M}^{\text {lump }}=\operatorname{diag}\left\{M_{A}\right\}, \quad M_{A}=\sum_{B=1}^{N_{\text {node }}} M_{A B},
$$

respectively. Then a smoothed mesh displacement field $\boldsymbol{d}^{\text {new }}$ is given by:

$$
\boldsymbol{d}_{A}^{\text {new }}=M_{A}^{-1} \sum_{B=1}^{N_{\text {node }}} M_{A B} \boldsymbol{d}_{B},
$$

which is clearly a local nodal update because the shape functions are compactly supported. The formula (5.4) also applies to boundary nodes and represents an "averaging" process among neighboring nodal displacements, from which the name "averaging method" found in the literature [56]. Then we perform an additional correction pass to obtain the final (smoothed) mesh displacement field $\boldsymbol{d}^{\mathrm{LS}}$ :

$$
\begin{aligned}
& \boldsymbol{d}_{A}^{\mathrm{LS}}=\boldsymbol{d}_{A}^{\text {new }}+\left(\left(\boldsymbol{d}_{A}-\boldsymbol{d}_{A}^{\text {new }}\right) \cdot \boldsymbol{n}_{A}\right) \boldsymbol{n}_{A}, \quad \forall \chi_{A} \in \Gamma, \\
& \boldsymbol{d}_{A}^{\mathrm{LS}}=\boldsymbol{d}_{A}^{\text {new }}, \quad \forall \chi_{A} \in \Omega_{\boldsymbol{\chi}} \backslash \Gamma .
\end{aligned}
$$

The $\boldsymbol{n}_{A}$ in $5.5 \mathrm{a}$ is the unit outer normal vector at $\chi_{A} \in \Gamma$, and this formula ensures that the nodal motion is tangential to the boundary during the smoothing. The new mesh position $\boldsymbol{x}_{n+1}$ is given by:

$$
x_{n+1}=x_{n}+d^{\mathrm{LS}} .
$$

Remark: The computational cost of the Laplacian smoothing algorithm resides in the assembly of the mass matrices (5.3) and the nodal updates (5.4). Because one typically reuses the computation of mass matrices when evolving the continuity equation in standard flow computations, the only additional cost associated with this algorithm are the nodal updates 5.4 . Hence, the Laplacian smoothing is highly efficient, even if multiple passes are used.

\subsection{A pseudo-linear-elasticity algorithm}

The Laplacian smoothing mesh motion algorithm is our preferred choice, since it tends to automatically refine the mesh resolution near shock fronts regardless of boundary conditions. This method, however, is also prone to over-coarsening the grid away from shock regions, jeopardizing at times the overall accuracy of the solution. For this reason, we also consider as an alternative a widely used pseudo-elasticity technique [57, 58] to deform the mesh.

In this method, $\boldsymbol{d}=\boldsymbol{x}_{n+1}-\boldsymbol{x}_{n}$ is treated as the displacement field in a quasi-static solid mechanics fictitious problem, with the undeformed material position given by $\boldsymbol{x}_{n}$. Introducing $\lambda$ and $\mu$ as fictitious Lamé's elasticity parameters, and solving the static linear elasticity equation

$$
\nabla_{\boldsymbol{x}_{n}} \cdot\left(\lambda \nabla_{\boldsymbol{x}_{n}} \cdot \boldsymbol{d} \boldsymbol{I}+\mu\left(\nabla_{\boldsymbol{x}_{n}} \boldsymbol{d}+\left(\nabla_{\boldsymbol{x}_{n}} \boldsymbol{d}\right)^{T}\right)\right)=0
$$

yields the deformed configuration of the grid. We complement the elliptic equation 5.7 by the boundary conditions:

$$
\begin{aligned}
\boldsymbol{d} & =\boldsymbol{x}^{\mathrm{corr}}-\boldsymbol{x}_{n}, \quad \boldsymbol{x}_{n} \in \Gamma \backslash \Gamma_{\boldsymbol{v}}, \\
\boldsymbol{d} \cdot \boldsymbol{n} & =\left(\boldsymbol{x}^{\mathrm{corr}}-\boldsymbol{x}_{n}\right) \cdot \boldsymbol{n}, \quad \boldsymbol{x}_{n} \in \Gamma_{\boldsymbol{v}} .
\end{aligned}
$$


Here $x^{\text {corr }}$ is the same as 5.2 1 The problem 5.7 - 5.8 leads to a linear solve with possible linear constraints if $\Gamma_{v}$ is not empty. We solve the resulting linear system using a preconditioned conjugate gradient algorithm.

Remark: It is possible to compute the two parameters $\lambda$ and $\mu$ adaptively in space to achieve certain computational targets (i.e., volume preservation). In this work we simply set $\lambda=\mu=1$.0. Interested readers may refer to [57] for a discussion of alternative strategies.

\subsection{Time-averaged versus time-interpolated mesh convective velocity}

Equation (3.4) is integrated in time from $t_{n}$ to $t_{n+1}$ assuming that the mesh position at both time instances is available. Namely, $\boldsymbol{x}_{n}$ is the solution from the previous time step, and $\boldsymbol{x}_{n+1}$ is computed using the method in this section. Specifically, we assume linear mesh motion in the time interval $\mathcal{I}=\left[t_{n}, t_{n+1}\right]$, and compute the instantaneous mesh position as

$$
\boldsymbol{x}_{n+\tau}=(1-\tau) \boldsymbol{x}_{n}+\tau \boldsymbol{x}_{n+1}
$$

with $\tau \in[0,1]$. Thus the mesh velocity is constant in each time interval, namely $\hat{\boldsymbol{v}}=\left(\boldsymbol{x}_{n+1}-\boldsymbol{x}_{n}\right) / \Delta t, \forall t \in \mathcal{I}$.

One advantage of the ALE framework is that when the mesh velocity is the same as the flow velocity, the equations reduce to the ones of Lagrangian dynamics. This property is also very desirable in the discretized equations, that is, the mesh convective velocity $\boldsymbol{c}=\boldsymbol{v}-\hat{\boldsymbol{v}}$ should automatically vanish for pure Lagrangian computations. However, this condition is not satisfied by the method presented in Sections 3.1 3.2. because $\hat{\boldsymbol{v}}$ is constant in each time step whereas neither $\boldsymbol{v}$ nor $\boldsymbol{v}^{h}$ are. To correct this situation, $v$ is replaced by $\overline{\boldsymbol{v}}^{h}$ in computations, where the latter is the flow velocity used in the Lagrangian mesh update procedure detailed in 5.1. Particularly, we have:

$$
\overline{\boldsymbol{v}}^{h}=\frac{1}{2}\left(\boldsymbol{v}_{n}^{h}+\boldsymbol{v}_{n+1}^{h}\right) .
$$

This modification affects only the calculation of $c^{h}$ and needs to be applied also at the boundary of the computational domain. The numerical Lagrangian computations at the end of this article show that using the time-interpolated mesh convective velocity $c^{h}=\boldsymbol{v}^{h}-\hat{v}$ leads to slightly more dissipative results than the time-averaged mesh convective velocity $c^{h}=\overline{\boldsymbol{v}}^{h}-\hat{\boldsymbol{v}}$.

\section{Time integration and other implementation aspects}

\subsection{A predictor/multi-corrector space-time method}

Time integration is performed using the explicit predictor multi-corrector algorithm discussed already in [8, 11, 23]. This method is based on a fixed-point iteration constructed with a space-time integrator, in which the solution is approximated as linear in time, and tested over function spaces that are constant in time. Full details and stability analysis are presented in [8]. For the sake of brevity, we omit the technical details, and we focus instead on specific aspects pertaining to the arbitrary Lagrangian-Eulerian framework. Algorithm 6.1 presents a summary of the timeintegration scheme, in which variables are updated following a Gauss-Seidel iteration.

\subsection{Temporal quadrature rules and the discrete geometric conservation law}

In the space-time algorithm that forms the basis to our explicit solver, the integration from $t_{n}$ to $t_{n+1}$ is performed using (3.4) and a temporal quadrature rule:

$$
\int_{0}^{1} f(\tau) d \tau \approx \sum_{q=1}^{n_{q}} w_{q} f\left(\tau_{q}\right), \quad \sum_{q=1}^{n_{q}} w_{q}=1 .
$$

The evaluation of the right hand side at any intermediate time $t_{n+\tau_{q}}=t_{n}+\tau_{q} \Delta t$ is performed by linear interpolation. For example, the flow density at any $t_{n+\tau_{q}}$ corresponding to the iteration $(i)$ is computed by:

$$
\rho_{n+\tau_{q}}^{(i), h}=\left(1-\tau_{q}\right) \rho_{n}^{h}+\tau_{q} \rho_{n+1}^{(i), h} .
$$

\footnotetext{
${ }^{1}$ Note that we only need to compute the corrected locations for the boundary nodes.
} 


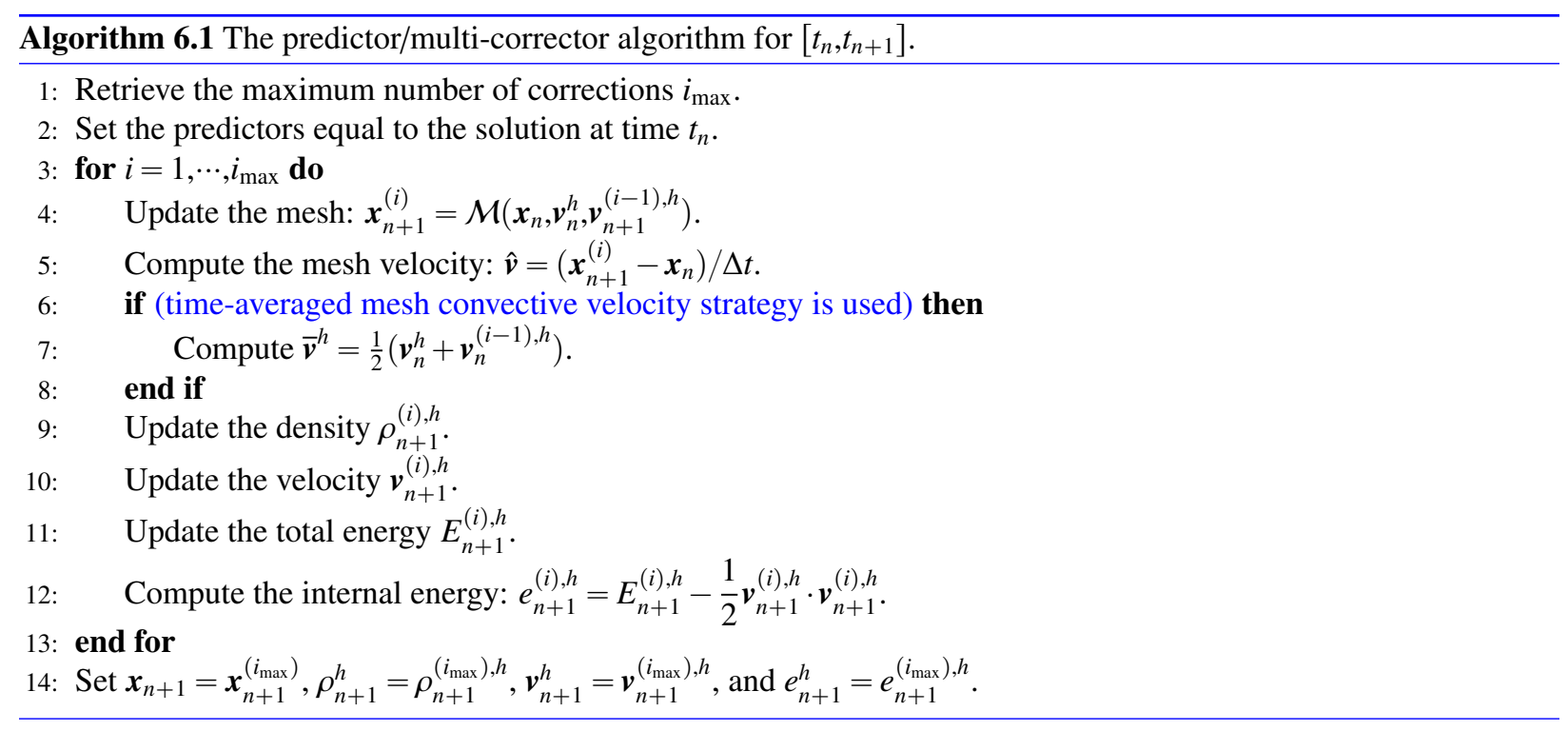

The same interpolation rule holds for mesh coordinates $\boldsymbol{x}_{n+\tau_{q}}$ and other flow variables.

The discrete geometric conservation law (DGCL) is well-known to be a desired property for ALE computations [11, 31, 59, 60]. In our context, the DGCL property can be satisfied by choosing appropriate temporal quadrature rules $\left\{w_{q}, \tau_{q}: q=1, \cdots, n_{q}\right\}$. Consider a special problem, for which the exact solution is the uniform flow with density $\rho=\rho_{0} \equiv 1$ (in both space and in time). Then we would expect the variational equations to be satisfied exactly for all test functions. In the case of the continuity equation, this requirement reads:

$$
\left.\int_{t_{n}}^{t_{n+1}} \int_{\Omega_{\chi}} \frac{\partial \hat{J}}{\partial t}\right|_{\chi} d \chi d t-\Delta t \sum_{q=1}^{n_{q}} w_{q} \int_{\Omega_{\chi}} \hat{J} \nabla \cdot \hat{v} d \chi=0 .
$$

For the linear mesh motion (5.9), a temporal integration rule with order $n_{d}$ is sufficient in order to satisfy the DGCL property. Hence, in our computations, we use:

$$
n_{q}=1, \quad w_{1}=1, \quad \tau_{1}=\frac{1}{2}
$$

for $n_{d} \leqslant 2$, and

$$
n_{q}=2, \quad w_{1,2}=\frac{1}{2}, \quad \tau_{1,2}=\frac{1}{2} \pm \frac{1}{2} \sqrt{\frac{1}{3}}
$$

for $n_{d}=3$.

\subsection{Computing the time-step size}

The time-step size in explicit dynamics is computed using a nominal Courant number. In particular, let $h_{e}^{\mathrm{cfl}}$ be the reference size of the element $\Omega_{e}$ to compute $\Delta t$, a conservative statement of the local Courant-Friedrich-Levy is:

$$
\sup _{x \in \Omega_{e}}\left(c_{s}^{h}+\left\|c^{h}\right\|+\frac{2 v_{\mathrm{art}}^{h}}{h_{e}^{\mathrm{cfl}}}\right) \Delta t \leqslant \alpha_{\mathrm{cfl}} h_{e}^{\mathrm{cfl}},
$$

where $v_{\mathrm{art}}^{h}$ is the artificial viscosity for the same element and the supremum is again taken as the maximum of the value at each node in the element. In principle, we should also consider the effects of the mass viscosity $\varepsilon_{\text {art }}$, however, (4.7p and (4.8) imply that $\varepsilon_{\text {art }}$ is always smaller than $v_{\text {art }}$. Thus we are safe to ignore the artificial mass viscosity in (6.5).

Applying the condition $\alpha_{\mathrm{cfl}} \leqslant 1, \sqrt[6.5]{ }$ motivates a conservative estimate of the element size $h_{e}^{\mathrm{cfl}}$ using the diameter of the circle or sphere inscribed into that element. Typically we have that $h_{e}^{\text {cfl }} \leqslant h_{e}$, where we recall that $h_{e}$ is the length scale in the stabilization terms. 
The time-step size computed according to a nominal Courant number $\alpha_{\mathrm{cfl}}$ is given by:

$$
\Delta t=\alpha_{\mathrm{cfl}} \min _{\Omega_{e} \subset \Omega} \frac{h_{e}^{\mathrm{cfl}}}{\sup _{x \in \Omega_{e}}\left(c_{s}^{h}+\left\|\boldsymbol{c}^{h}\right\|+2 v_{\mathrm{art}}^{h} / h_{e}^{\mathrm{cfl}}\right)} .
$$

A typical range of the Courant number for low-order explicit time-integrators, such as the first-order forward Euler, second-order Runge-Kutta, and the predictor/multi-corrector method, is $\alpha_{\mathrm{cff}} \in[0,1]$. In ALE computations, because the element in general deforms from one time step to the next, the reference element size $h_{e}^{\text {cfl }}$ also varies from time step to time step. In our computations we use a conservative value $\alpha_{\mathrm{cfl}}=0.8$ for all the numerical examples and experience no instability at all.

Remark: When only two corrector passes are performed, the proposed method corresponds to a TVD RK2 integrator (total-variation diminishing, second-order, two-stage Runge-Kutta method). In general, however, predictor/multicorrector time-integrators are less common in Eulerian computations, comparing to explicit Runge-Kutta methods. We choose this specific time-integrator mainly for its efficiency (low storage) and its ability to preserve the angular momentum in the limit $i_{\max } \rightarrow \infty$ [23], which proves to be important for Lagrangian computations. The proposed methodology can be extended to integrators other than the TVD-RK2. Low-storage Runge-Kutta methods [61] can be obtained by choosing the iteration number $i_{\max }$ and the coefficients $w_{q}$ and $\tau_{q}$ appropriately without adding to the storage burden.

\section{Numerical examples}

In this section, we present the numerical assessment of the proposed method. We begin by studying the accuracy of the method in Section 7.1. The capability of the monolithic ALE solver of handling mixed Lagrangian and Eulerian boundary conditions are presented in Section 7.2 , the stability of the method in computing with very strong shocks is demonstrated by blast wave simulations in Section 7.3 , and, finally, a three-dimensional example is offered in Section 7.4 .

For all the computations, we use the predictor/multi-corrector time-integrator with $i_{\max }=4$ (i.e., one more iterate with respect to a TVD-RK2 method) and fixed Courant number $\alpha_{\mathrm{cfl}}=0.8$. The VMS-stabilization parameter is fixed to $c_{\tau}=2.5$. Unless otherwise specified, we fix the entropy viscosity constant to $c_{\text {ent }}=40.0$. We also indicate in the title of each section which mesh motion strategies are considered for the particular numerical example.

\subsection{The two-dimensional Taylor-Green vortex (Eulerian, ALE with Laplacian smoothing, Lagrangian)}

We consider $c_{\text {ent }}=10.0$ and $c_{\text {ent }}=40.0$ in this section to assess the accuracy of the method. Both values demonstrate second-order convergence in all flow variables when the grids are sufficiently refined. The two-dimensional TaylorGreen vortex has a smooth and time-independent solutions [22]. This is achieved by adding a manufactured source of energy $\rho r$ :

$$
\left.\frac{\partial \rho E}{\partial t}\right|_{\chi}+\boldsymbol{c} \cdot \nabla(\rho E)+(\nabla \cdot v) \rho E+\nabla \cdot(p \boldsymbol{v})-\rho r=0 .
$$

Accordingly, we modify the discrete residuals for internal energy, pressure, and entropy as:

$$
\begin{aligned}
& \operatorname{Res}_{e}^{h}=\left.\frac{\partial e^{h}}{\partial t}\right|_{\chi}+c^{h} \cdot \nabla e^{h}+\frac{\partial p^{h}}{\partial \rho^{h}} \nabla \cdot v^{h}-r^{h}, \\
& \operatorname{Res}_{p}^{h}=\left.\frac{\partial p^{h}}{\partial t}\right|_{\chi}+\boldsymbol{c}^{h} \cdot \nabla p^{h}+\rho^{h} c_{s}^{h 2} \nabla \cdot \boldsymbol{v}^{h}-r^{h} \frac{\partial p}{\partial e}\left(\rho^{h}, e^{h}\right), \\
& \operatorname{Res}_{s}^{h}=\left.\frac{\partial s^{h}}{\partial t}\right|_{\chi}+\boldsymbol{c}^{h} \cdot \nabla s^{h}+s^{h} \nabla \cdot v^{h}-\rho^{h} r^{h}\left(\rho^{h} / p^{h \gamma}\right)^{\frac{1}{\gamma+1}} .
\end{aligned}
$$

For ideal gases, the last term in the pressure residual simplifies to $p_{e}\left(\rho^{h}, e^{h}\right)=(\gamma-1) \rho^{h}$. The initial conditions as well as the exact solutions for the Taylor-Green vortex are:

$$
\rho(\boldsymbol{x}, t)=1, \quad \boldsymbol{v}(\boldsymbol{x}, t)=(\sin (\pi x) \cos (\pi y),-\cos (\pi x) \sin (\pi y)), \quad p(\boldsymbol{x}, t)=\frac{1}{4}(\cos (2 \pi x)+\cos (2 \pi y))+1,
$$




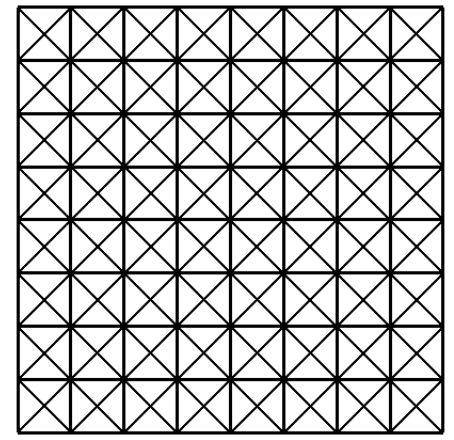

(a) Eulerian.

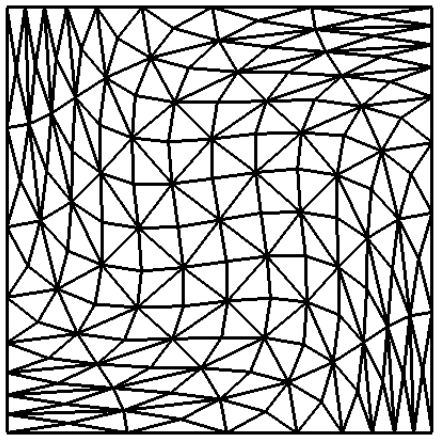

(b) ALE with Laplacian smoothing.

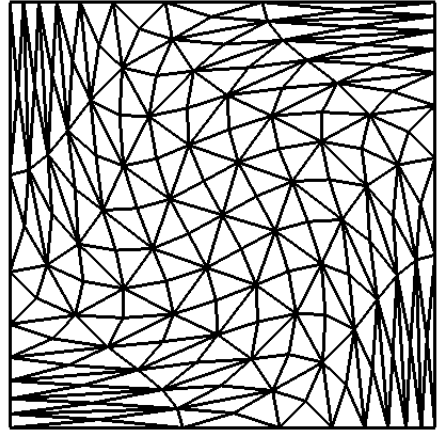

(c) Lagrangian.

Figure 7.1: The final positions of a $4 \times 8^{2}$ grid at $T=0.5$ by various mesh motion strategies for the Taylor-Green vortex.

on the domain $\Omega=[0,1]^{2}$. The specific heat ratio is $\gamma=5 / 3$ and the heat source is

$$
r(\boldsymbol{x}, t)=\frac{3 \pi}{8}(\cos (3 \pi x) \cos (\pi y)-\cos (\pi x) \cos (3 \pi y)) .
$$

We solve the problem using structured triangular grids, which are obtained by subdividing each cell of a uniform quadrilateral grid into four triangles of equal size. At the terminal time $T=0.5$, the final $4 \times 8^{2}$ mesh position is plotted in Figure 7.1 for computations of Eulerian, ALE (Laplacian smoothing), and Lagrangian type computations.

We plot the $L_{2}$-errors in density, velocity, internal energy, and total energy on a sequence of seven grids in Figure 7.2 and Figure 7.3 for the two choices $c_{\mathrm{ent}}=10.0$ and $c_{\mathrm{ent}}=40.0$, respectively. These grids have the number of elements ranging from $4 \times 4^{2}$ to $4 \times 256^{2}$. On the coarsest grids, the convergence rate is slow because the von Neumann viscosity is smaller than the entropy viscosity, whereas as soon as the grid size reaches $4 \times 16^{2}$, all the methods show second-order convergence in all flow variables.

Finally, because no flow particles enter or exit the computational domain, the Taylor-Green vortex problem is excellent to investigate the entropy conservation property of the proposed method. Because we do not directly test the transport equation for the entropy $s$, that is 4.2, exact conservation cannot be expected numerically, although this is the case analytically. In Figure 7.4a and Figure $7.4 \mathrm{~b}$ for two values of $c_{\text {ent }}$, we plot the history of the total entropy. In particular, as we refine the grids we observe that: (1) the initial total entropy converges to the same value, (2) the growth of the total entropy in time becomes negligible, and (3) larger $c_{\text {ent }}$ lead to larger growth rates of entropy for a fixed grid size.

We can draw the following conclusions on the accuracy of the proposed method from previous results:

1. When the mesh is sufficiently fine, the proposed method is formally second-order accurate in all flow variables regardless of the mesh motion strategy utilized.

2. The method tends to converge slowly on coarse grids, because the lower-order von Neumann viscosities are more likely to be active on these grids.

3. On finer grids, $v_{\text {ent }}$ becomes active and the method shows second-order convergence.

4. For a smaller value of $c_{\text {ent }}$ the second-order asymptotic convergence range is reached sooner.

5. The total entropy is conserved up to an error that vanishes when the mesh is refined.

\subsection{Channel flows}

\subsubsection{Flow past a Ni-bump (Eulerian)}

The Ni-bump problem [62] is a slightly more complicated channel flow problem, in which a small bump is placed on the bottom of the channel. This is a well accepted test for Eulerian computations of shocks with weak to moderate 


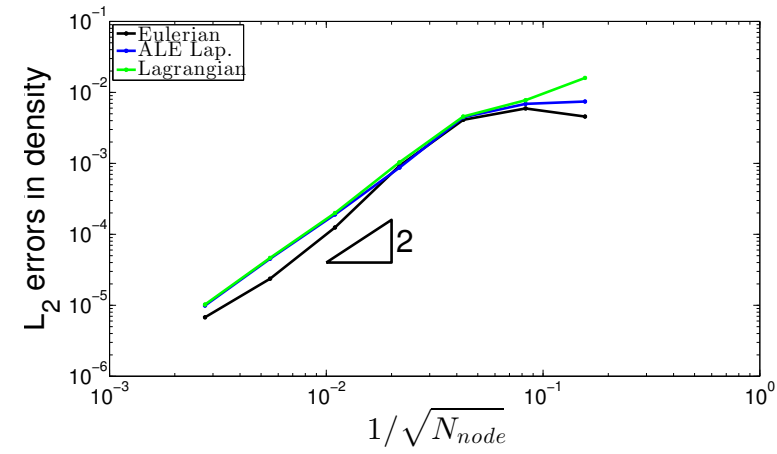

(a) $L_{2}$-errors in density.

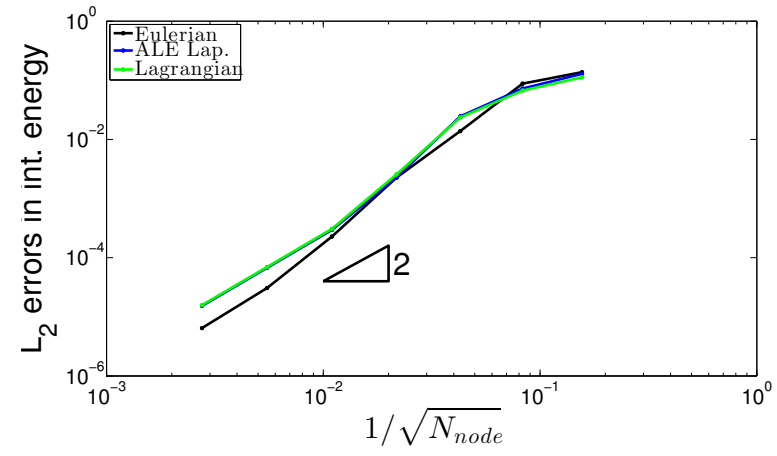

(c) $L_{2}$-errors in internal energy.

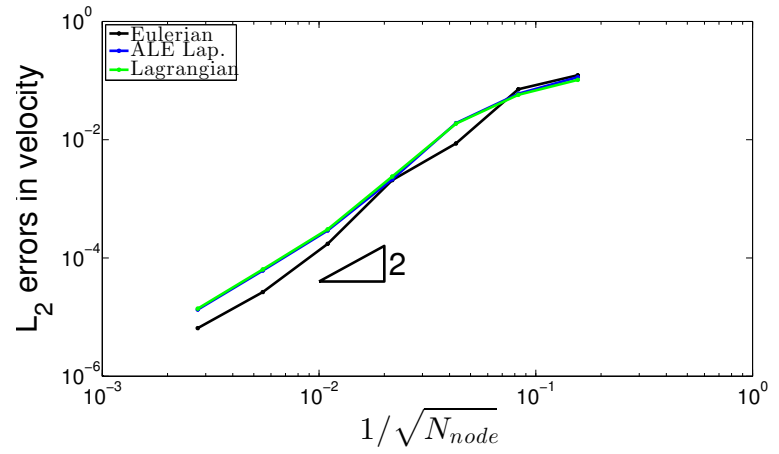

(b) $L_{2}$-errors in velocity.

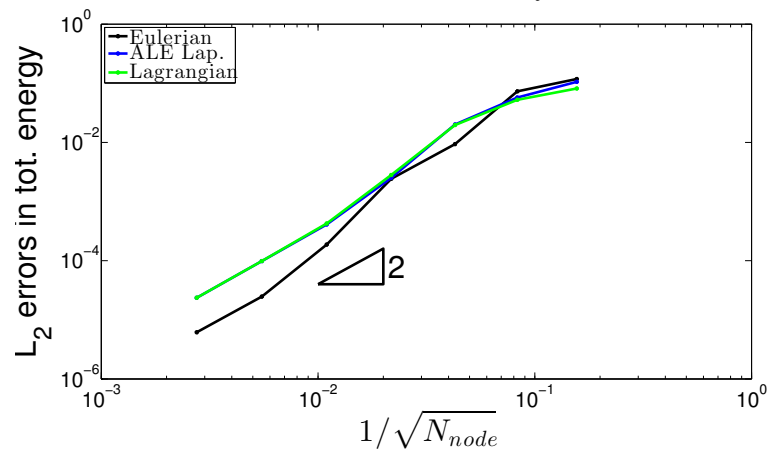

(d) $L_{2}$-errors in total energy.

Figure 7.2: $L_{2}$-errors in $\rho^{h}, v^{h}, e^{h}$, and $E^{h}$ vs. $1 / \sqrt{N_{\text {node }}}$ for the two-dimensional Taylor-Green vortex. The artificial viscosity parameter is set to $c_{\text {ent }}=10.0$. The blue curves, the black curves, and the green curves correspond to the Eulerian tests, the ALE tests with Laplacian-smoothing, and the Lagrangian tests, respectively. 


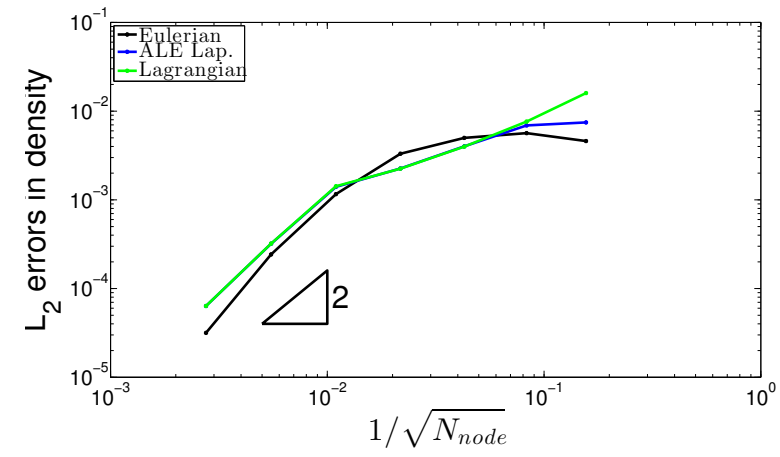

(a) $L_{2}$-errors in density.

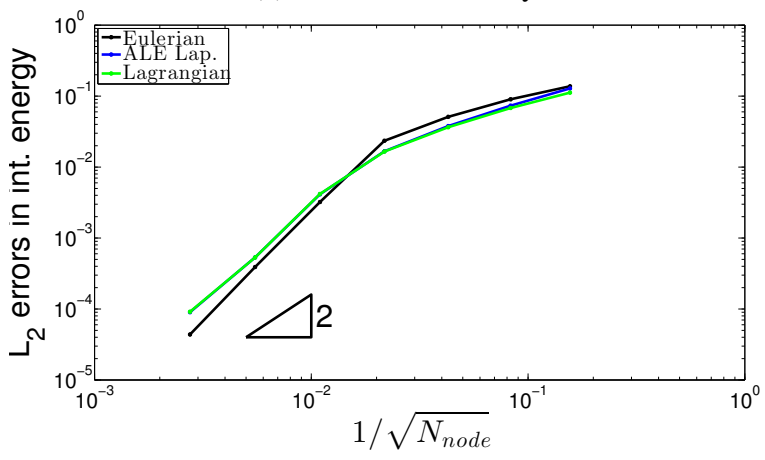

(c) $L_{2}$-errors in internal energy.

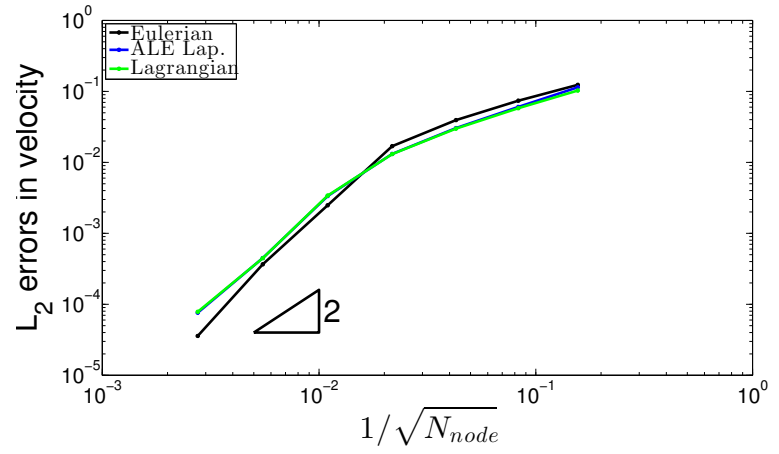

(b) $L_{2}$-errors in velocity.

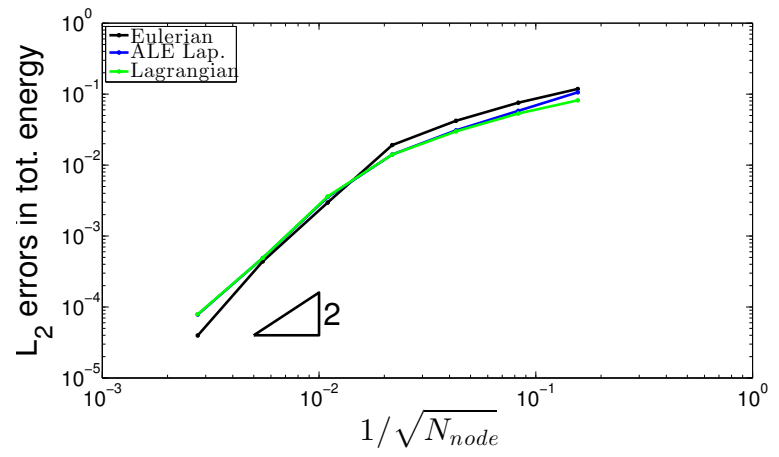

(d) $L_{2}$-errors in total energy.

Figure 7.3: $L_{2}$-errors in $\rho^{h}, v^{h}, e^{h}$, and $E^{h}$ vs. $1 / \sqrt{N_{\text {node }}}$ for the two-dimensional Taylor-Green vortex. The artificial viscosity parameter is set to $c_{\text {ent }}=40.0$. The blue curves, the black curves, and the green curves correspond to the Eulerian tests, the ALE tests with Laplacian-smoothing, and the Lagrangian tests, respectively.
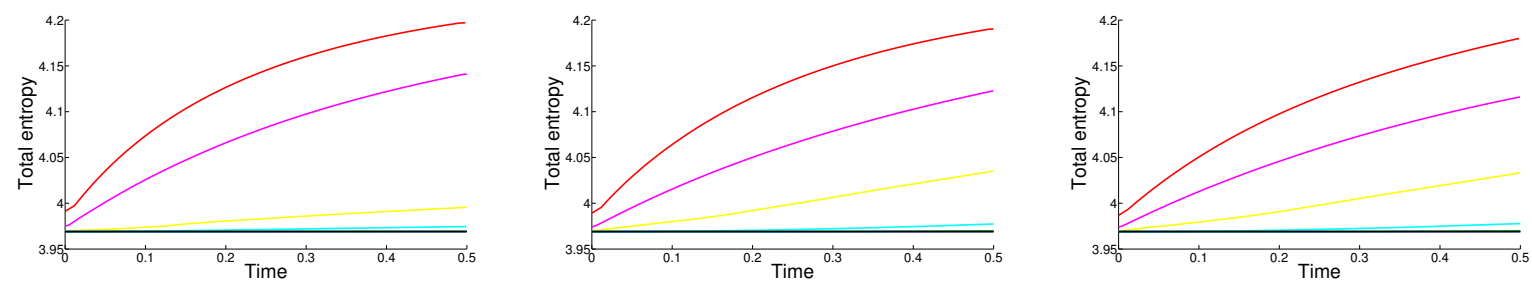

(a) $c_{\text {ent }}=10.0$, from left to right: Eulerian, ALE, and Lagrangian.
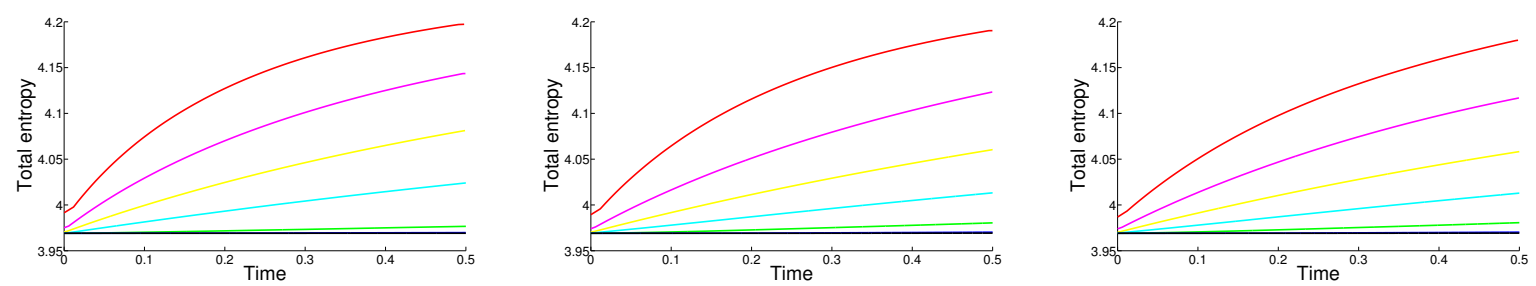

(b) $c_{\text {ent }}=40.0$, from left to right: Eulerian, ALE, and Lagrangian.

Figure 7.4: Two-dimensional Taylor-Green vortex: Total entropy vs. time using different entropy viscosity constants $c_{\text {ent }}$. Different colors represent different grids: $4 \times 4^{2}$ (red), $4 \times 8^{2}$ (magenta), $4 \times 16^{2}$ (yellow), $4 \times 32^{2}$ (cyan), $4 \times 64^{2}$ (green), $4 \times 128^{2}$ (blue), and $4 \times 256^{2}($ black) . 


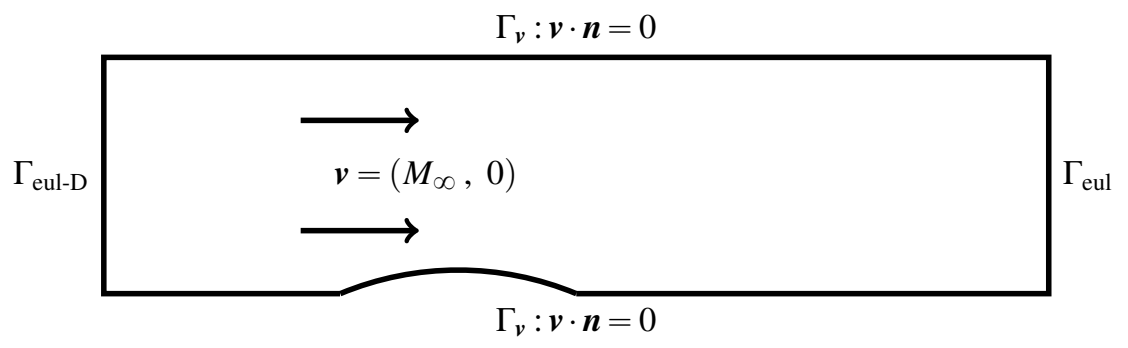

Figure 7.5: The computational domain for the Ni-bump problem.

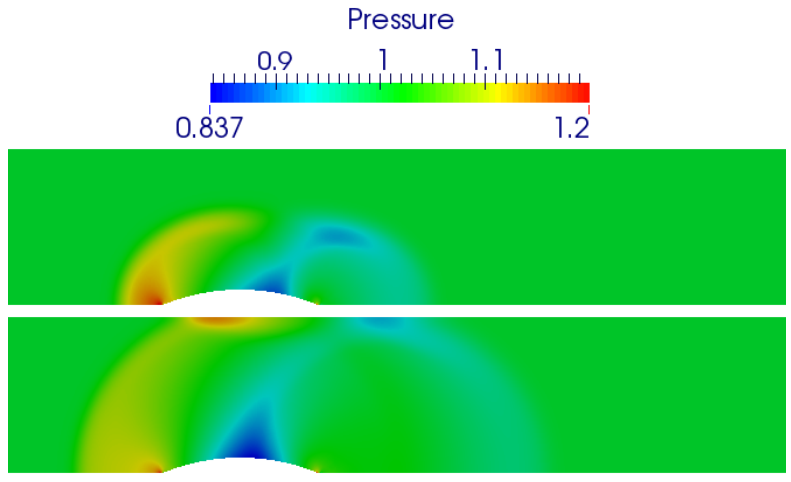

(a) Pressure.

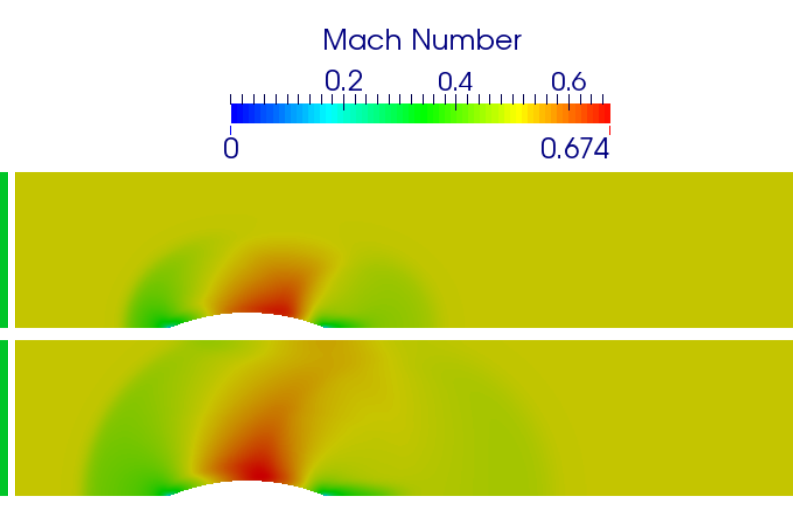

(b) Mach number.

Figure 7.6: Pressure and Mach number for a subsonic flow past a Ni-bump at $t=0.5$ (top) and $t=1.0$ (bottom). Numerical solutions use VMSstabilization $c_{\tau}=2.5$ and entropy viscosity $c_{\text {ent }}=40.0$.

strength, caused by the flow deflection at the trailing edge of the bump. Figure 7.5 depicts the problem, where a rectangular domain of size $1 \times 4$ contains a bump in the shape of an arc of radius 1.3 and height 0.1 . The mid-point of the bump span is located 1.5 downstream of the left domain boundary. The initial condition is given by ideal gas with $\gamma=1.4$ and

$$
\rho_{0}=1.4, \quad \boldsymbol{v}=\left(M_{\infty}, 0.0\right), \quad p=1.0,
$$

where $M_{\infty}$ is the free-stream Mach number. Inflow boundary conditions are specified at the left boundary, outflow boundary conditions at the right boundary, and zero normal velocity boundary condition on all other edges (upper, lower, and the bump). Note that the flow velocity is forced to be zero at the leading edge and the trailing edge of the bump.

The domain is subdivided into 71,072 unstructured triangles and 36,018 nodes, and computations are performed until time $T=1.0$. Because the time-interpolated and the time-averaged convection velocities lead to similar results, we only report the solutions with the latter.

We present the pressure and Mach number at $t=0.5,1.0$ for a subsonic channel flow with free-stream Mach number $M_{\infty}=0.5$ in Figure 7.6 Next, we increase the free-stream flow velocity to $M_{\infty}=2.0$, and plot the pressure and Mach number for this supersonic channel flow in Figures 7.7. The computational results show that the flow stays smooth and numerically stable throughout computations.

\subsubsection{Flow/shock past a stationary block (Eulerian)}

A more challenging problem is the flow past a square blockage, because of the singularities in the geometry. The computational domain is a rectangle of size $1 \times 5$, with a square $0.4 \times 0.4$ block, whose lower left (point A) is located at a distance of 1.4 downstream of the inlet. First we consider a supersonic flow, depicted in Figure 7.8 . Slip boundary conditions are specified at the upper and lower boundaries (including the sides of the block), and the flow velocity at 


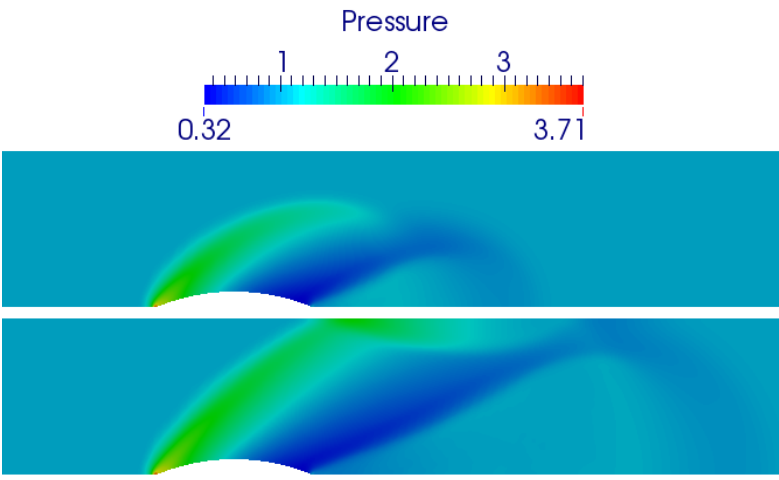

(a) Pressure.
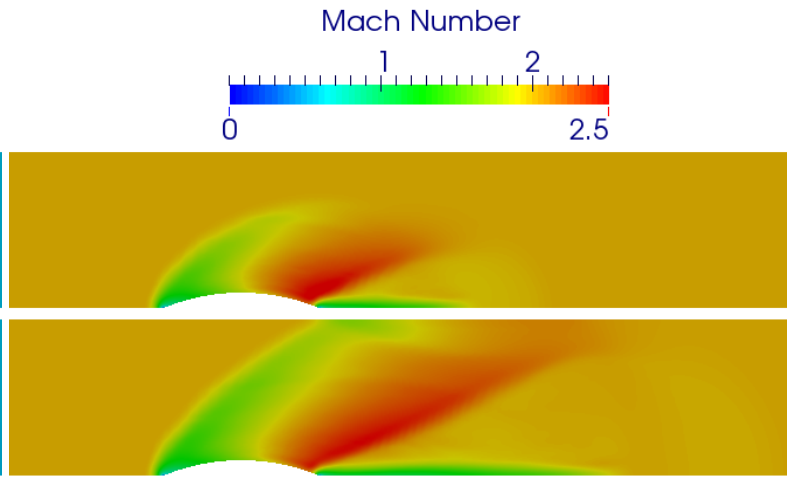

(b) Mach number.

Figure 7.7: Pressure and Mach number for a supersonic flow past a Ni-bump at $t=0.5$ (top) and $t=1.0$ (bottom). Numerical solutions use VMS-stabilization $c_{\tau}=2.5$ and entropy viscosity $c_{\mathrm{ent}}=40.0$.

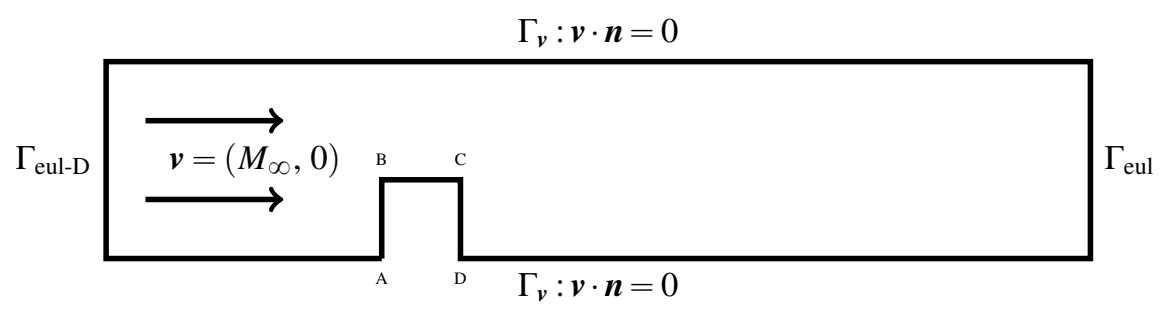

Figure 7.8: The computational domain for the uniform supersonic flow past a stationary block.

the four corners of the block (A, B, C, and D in the figure) is set to zero at all time. The left boundary designates an inflow boundary, and the right boundary is an outflow boundary.

In the first test, we consider a uniform flow with Mach number $M_{\infty}=1.4$ passing the block. The initial condition is a uniform ideal gas throughout the domain: $\rho_{0}=1.4, p_{0}=1.0, v=\left(M_{\infty}, 0.0\right)$, and $\gamma=1.4$. We solve the problem on a unstructured grid with 111,672 triangles and 56,477 nodes. The terminal time of the simulation is $T=3.6$, just before the wave generated by the reflection at the point B reaches the left boundary, so that our inflow boundary remains valid throughout the computation. We demonstrate the development of the flow pressure and Mach number (at $t=1.2$, 2.4, and 3.6) in Figures 7.9 .

Next we consider a similar problem, in which the initial condition is given by a right-moving planar shock instead of the uniform flow. For a relatively weak shock (Mach 1.2), we consider the same computational domain and the block of the same size as before, but this time the block is located at the center of the bottom of the channel, as shown in Figure 7.10 . The pre-shock (on the right of the shock front) flow variables are

$$
\rho_{\text {pre }}=1.4, \quad p_{\text {pre }}=1.0, \quad v_{\text {pre }}=(0.0,0.0),
$$

and the post-shock (to the left of the shock front) flow variables are

$$
\rho_{\text {post }}=1.878261, \quad p_{\text {post }}=1.513333, \quad v_{\text {post }}=(0.30556,0.0) .
$$

The flow is ideal gas with specific heat capacity ratio $\gamma=1.4$.

An unstructured triangular mesh of 111,612 elements and 56,447 nodes is used, and the terminal time of the simulation is $T=2.5$, when the reflected shock front at point B is about the reach the inlet boundary. The shock reaches the block right before $T=0.4$, and the flow development is plotted in Figures 7.11 for the pressure and Mach number at $t=1.0,1.5$, and 2.5 .

In the last example of this section, we increase the strength of the shock to Mach 5.0, and the block is moved upstream, so that the corner A is 1.0 downstream of the inlet, as shown in Figure 7.12 The pre-shock data remains 


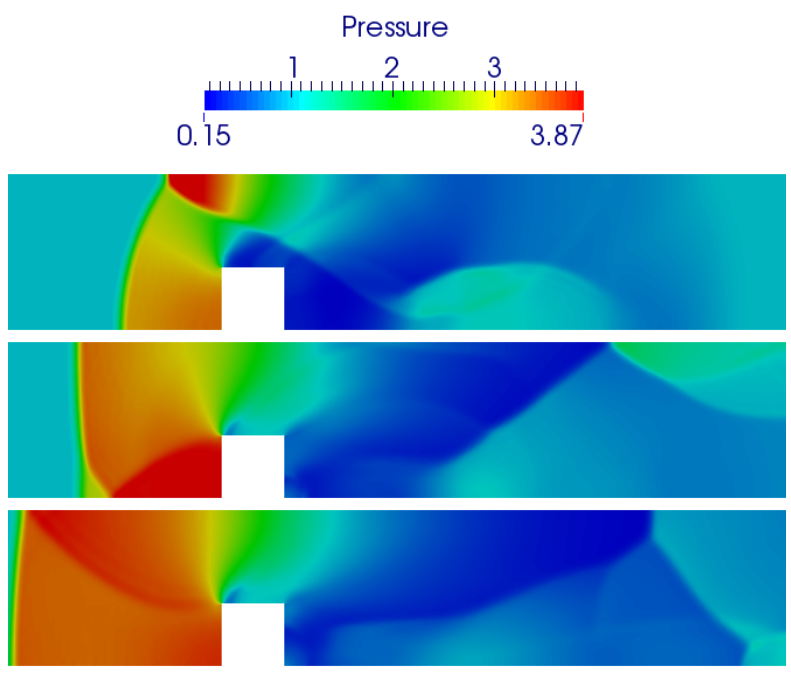

(a) Pressure.

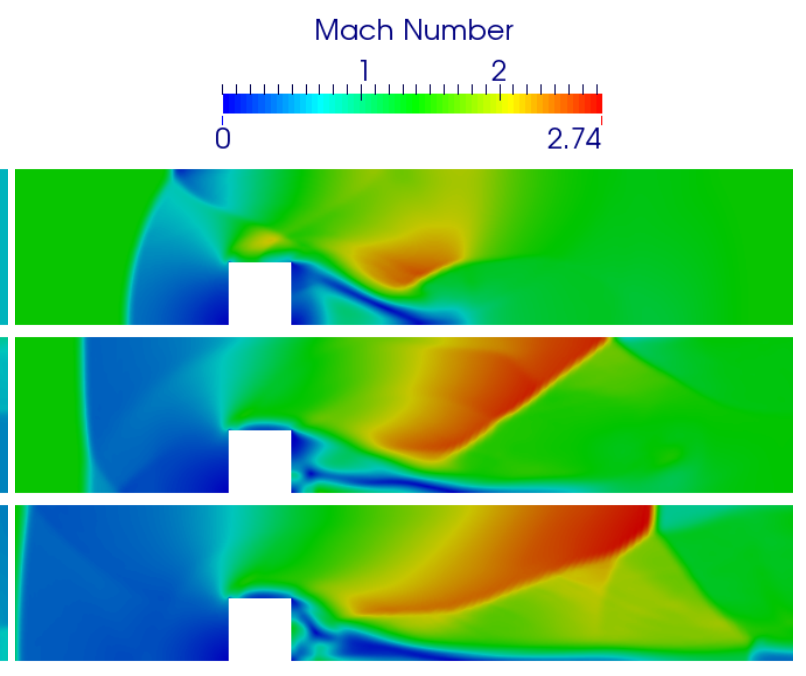

(b) Mach number.

Figure 7.9: Pressure and Mach number for a supersonic flow past a stationary block at time $t=1.2$ (top), $t=2.4$ (middle), and $t=3.6$ (bottom). Numerical solutions use VMS-stabilization $c_{\tau}=2.5$ and entropy viscosity $c_{\text {ent }}=40.0$.

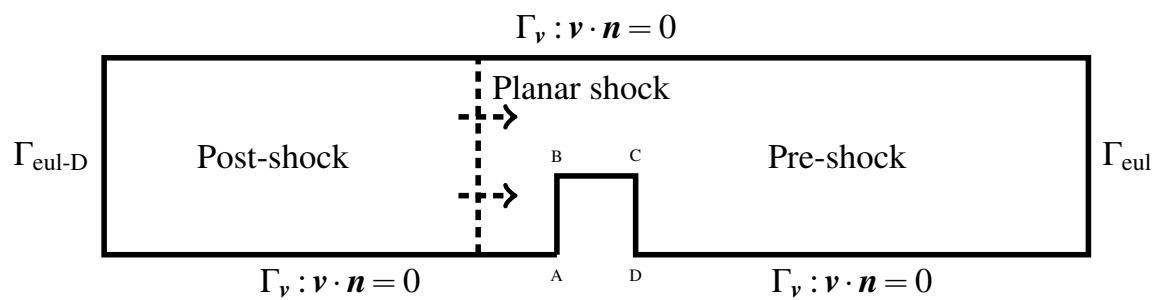

Figure 7.10: The computational domain for a Mach 1.2 planar shock past a stationary block.

Pressure
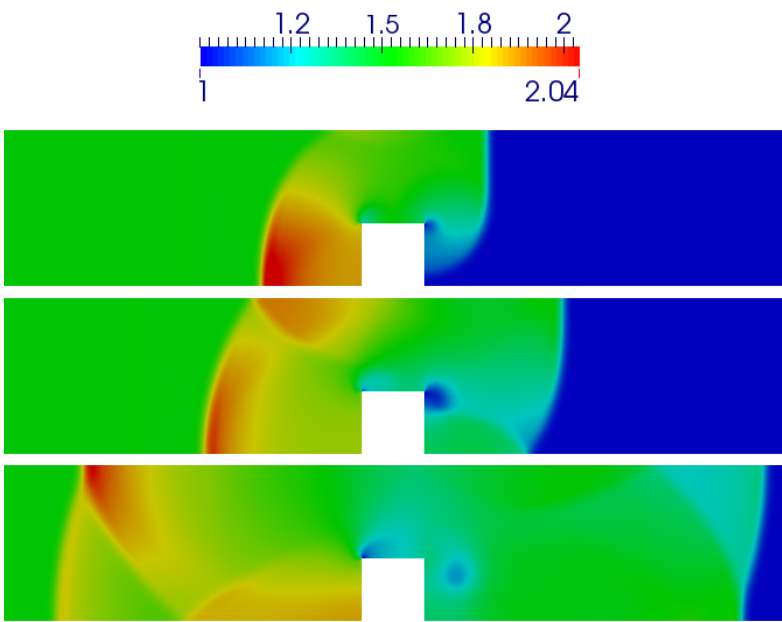

(a) Pressure.
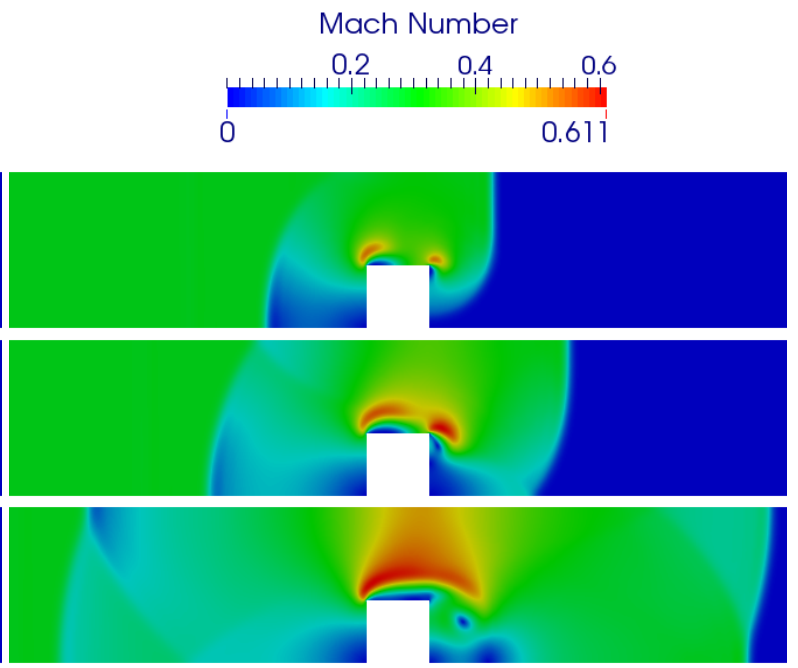

(b) Mach number.

Figure 7.11: Pressure and Mach number for a Mach 1.2 planar shock past a stationary block at time $t=1.0$ (top), $t=1.5$ (middle), and $t=2.5$ (bottom). Numerical solutions use VMS-stabilization $c_{\tau}=2.5$ and entropy viscosity $c_{\mathrm{ent}}=40.0$. 


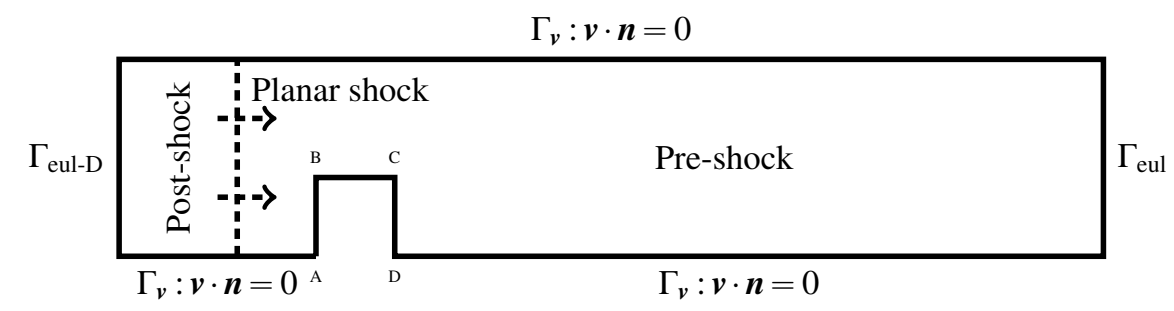

Figure 7.12: The computational domain for a Mach 5.0 planar shock past a stationary block.

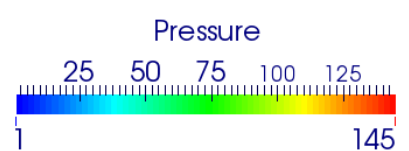

145
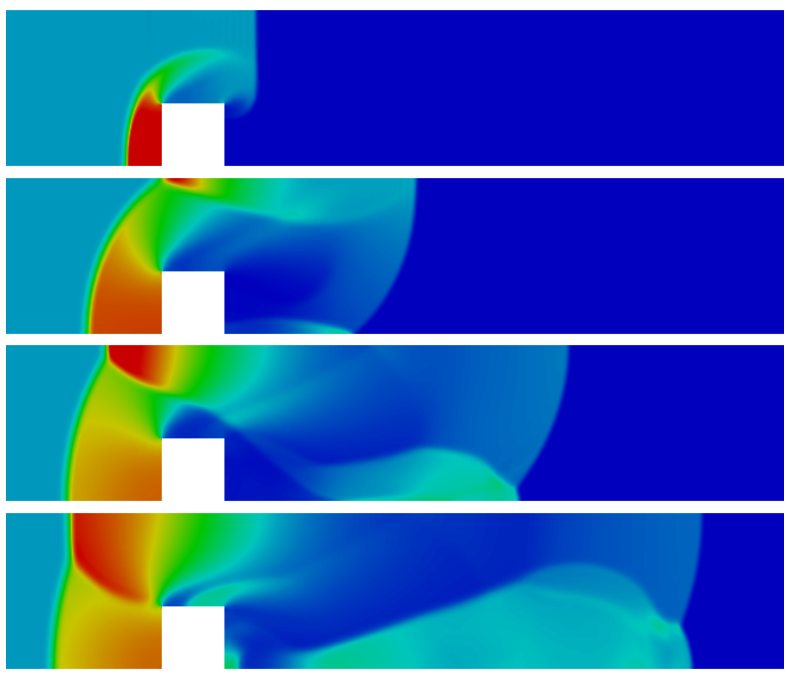

(a) Pressure.
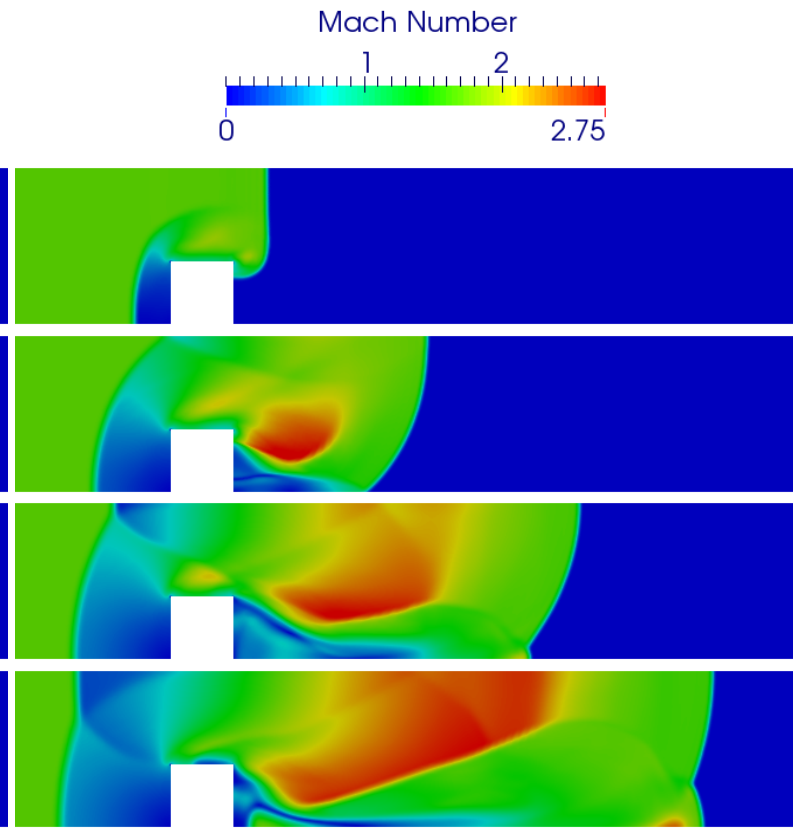

(b) Mach number.

Figure 7.13: Pressure and Mach number for a Mach 5.0 planar shock past a stationary block at time $t=0.2,0.4,0.6$, and 0.8 (from top to bottom). Numerical solutions use VMS-stabilization $c_{\tau}=2.5$ and entropy viscosity $c_{\text {ent }}=40.0$.

the same as (7.8), whereas the post-shock flow variables are

$$
\rho_{\text {post }}=7.0, \quad p_{\text {post }}=29.0, \quad v_{\text {post }}=(4.0,0.0) .
$$

We solve the problem until $T=0.8$, when the reflected shock is about to reach the inlet and we plot the flow pressure and Mach number at the temporal frequency 0.2 in Figures 7.13

We conclude from these computations that the chosen set of stabilization parameters $\left(c_{\tau}=2.5\right.$ and $\left.c_{\text {ent }}=40.0\right)$ work equally well for a wide range of strength of planar shocks.

\subsubsection{Nozzle flow (Eulerian, ALE with linear-elasticity mesh smoothing)}

The last example in this section is the flow passing through a nozzle (Figure 7.14). The computational domain is symmetric, and obtained by cutting two disks with radius 0.5 from a T-shape region. The dimensions are shown in Figure 7.14 The upper and lower edges (A-H-G-F and B-C-D-E) are fixed walls with slip wall boundary conditions. The initial flow is at rest in the nozzle, with density $\rho=1.4$ and pressure $p=1.0$. A uniform flow is injected at the left edge $(\mathrm{A}-\mathrm{B})$ of the nozzle starting from $t=0.0$, and the inflow has the following conditions:

$$
\rho_{A B}=1.4, \quad p_{A B}=1.96, \quad \boldsymbol{u}_{A B}=(0.5,0.0) .
$$




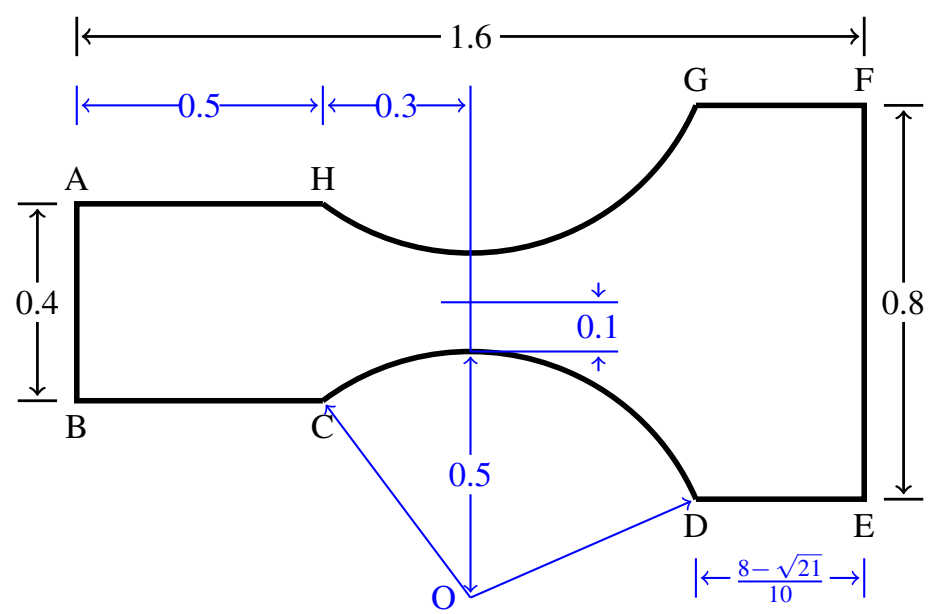

Figure 7.14: Initial computational domain of the nozzle. Edges A-H-G-F and B-C-D-E are stationary slip walls; the edge A-B is the inlet of the domain. For the Eulerian computation, the edge F-E is a fixed Eulerian boundary and for the ALE computation, the edge F-E has pressure boundary condition.

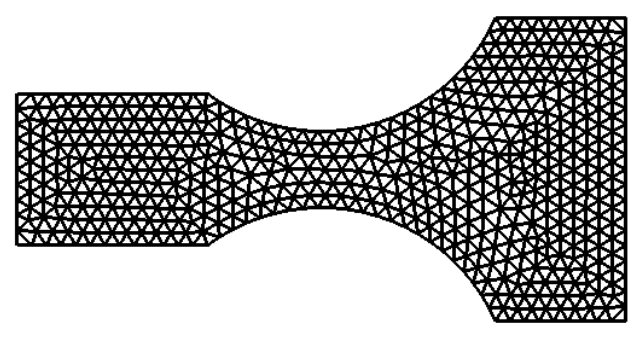

(a) The Eulerian mesh.

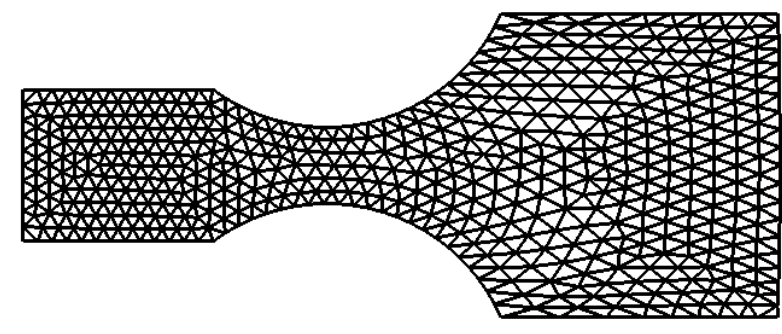

(b) Final ALE mesh at $T=1.8$.

Figure 7.15: Examples of the unstructured triangular grids for the nozzle test: 7.15 a the Eulerian grid and the initial grid for the ALE computation and $7.15 \mathrm{~b})$ the final ALE mesh at $T=1.8$.

We consider both an Eulerian computation and an ALE computation. In the former, the right boundary (E-F) is an outflow boundary, whereas for the ALE computation, a pressure boundary condition of $p=1.0$ is specified, and motion is allowed. Figure 7.15 shows mesh configurations at different stages of the computation for both cases.

We compute the flow in both tests until $T=1.8$ using an unstructured grid with 15,246 triangles and 7,858 nodes. The wave fronts traverses the center of the nozzle throat approximately at $t=0.6$ and reaches the initial location of the right boundary at about $t=1.2$. The solutions at these two times as well as the terminal time for both the Eulerian computation and the ALE computation are plotted in Figures 7.16 and 7.17 for the pressure and the Mach number, respectively. In all the computations, the time-averaged approach is utilized to compute the convection velocity. Note that to plot the solutions, we truncated the higher end of the ranges for both the pressure and the Mach number, which peak along the neck downstream corners $\mathrm{C}$ and $\mathrm{H}$, so that we may visualize more details of the flow patterns in other regions.

Before the traveling wave passes the original location of the outflow boundary, the Eulerian computation and the ALE computation must have the same solution. Past that point in time, this is not the case any longer. Up to the crossing time the Eulerian and ALE numerical solutions compare well with each other. When the wave front starts to interact with the right boundary of the domain, the two tests behave differently. In particular, in the ALE computation the flow interacts with a stationary gas with prescribed pressure whereas in the Eulerian computation the flow is allowed to exit freely. Hence we observe reflected waves in the ALE solutions (particularly at the bottom of Figure 7.16b, whereas these are absent from the Eulerian computations. 

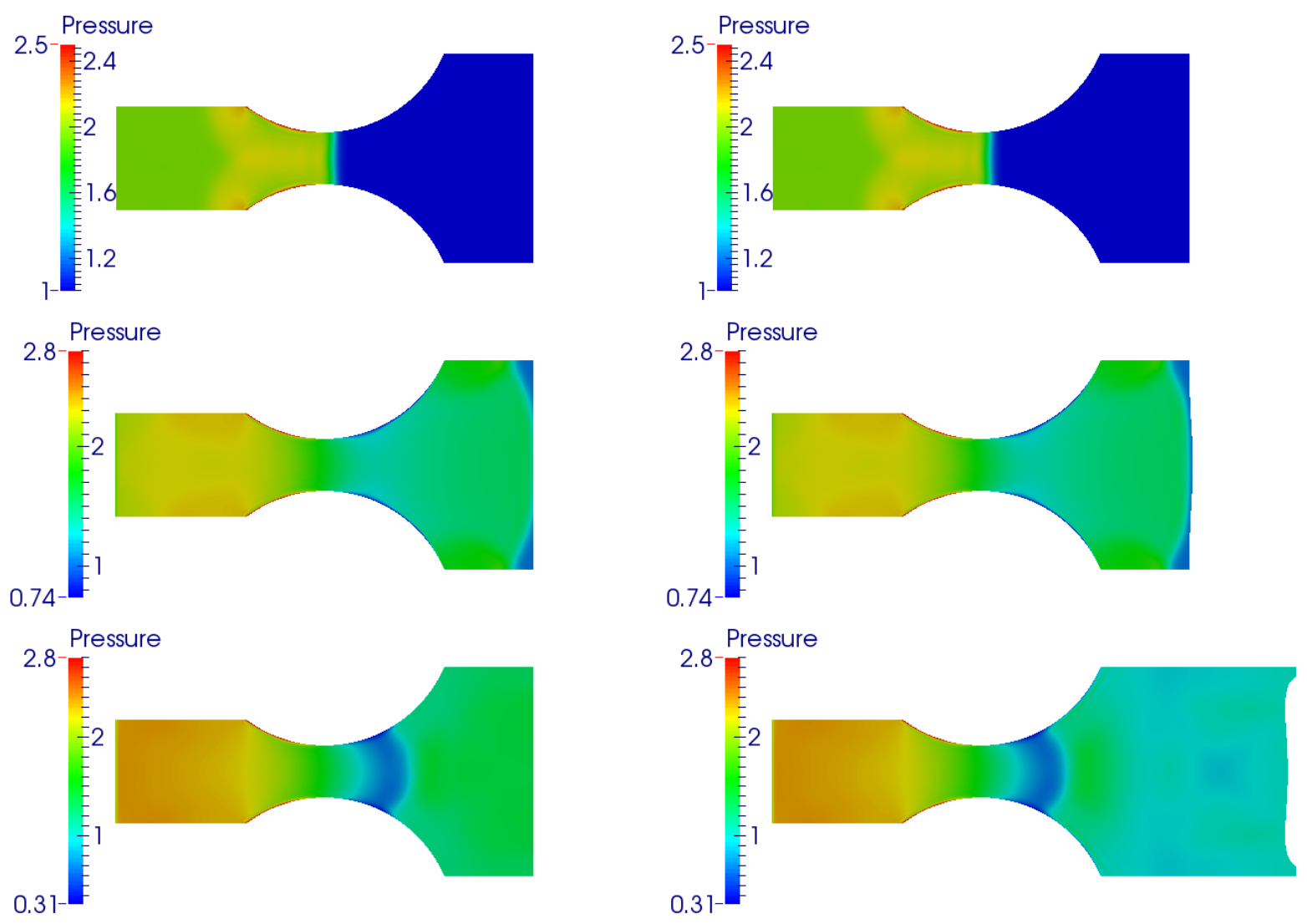

(a) Eulerian T-ave., pressure.

(b) ALE T-ave., pressure.

Figure 7.16: Nozzle problem: Evolution of the pressure. From top to bottom, $t=0.6,1.2$, and 1.8. Numerical solutions use VMS-stabilization $c_{\tau}=2.5$ and entropy viscosity $c_{\mathrm{ent}}=40.0$. 

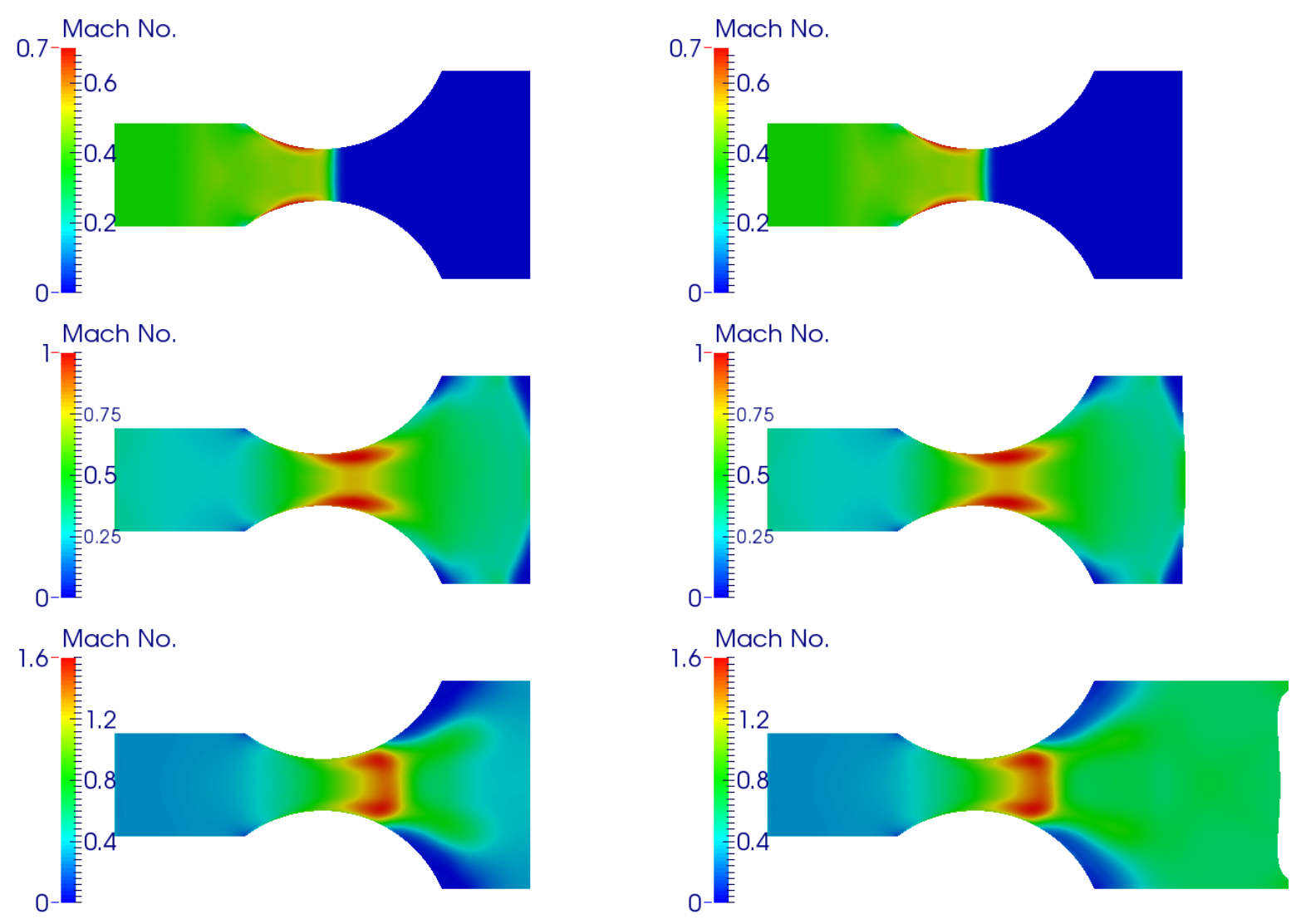

(a) Eulerian T-ave., Mach number.

(b) ALE T-ave., Mach number.

Figure 7.17: Nozzle problem: Evolution of the Mach number. From top to bottom, $t=0.6,1.2$, and 1.8. Numerical solutions use VMS-stabilization $c_{\tau}=2.5$ and entropy viscosity $c_{\mathrm{ent}}=40.0$. 


\subsubsection{Conclusions}

The numerical examples in this section share a few features: First, the numerical results show that the proposed method is very flexible in handling inflow and outflow boundary conditions, in addition to the conventional velocity boundary condition of Lagrangian shock hydrodynamics. Second, there are at least two types of singularities in the tests: (a) Geometrical singularities at corners, with rapid flow rotations and reflections, and (b) singularities produced by inconsistencies between initial and boundary conditions. Both types of singularity tend to create severe spurious oscillations or instabilities in flow computations, but our numerical results show that the proposed VMS-stabilization and entropy viscosity combine to handle these issues very well.

\subsection{Blast waves}

The blast wave test by Sedov [63] is a standard test for strong shocks. It describes the cylindrical symmetric shock (or spherical symmetric shock in three dimensions) initiated by a point source of high energy in a cold environment (i.e., nearly zero absolute temperature). The problem allows an exact solution that is easy to compute. We test the proposed monolithic ALE algorithm by solving the Sedov blast wave in various context and in different numerical settings.

\subsubsection{Sedov blast wave (Eulerian, ALE with Laplacian smoothing, Lagrangian)}

In this classical test, we have a square domain $[-1.1,1.1]^{2}$ with a concentrated Dirac distribution of internal energy at the domain center. Because of the symmetry of the problem, we only simulate the blast wave in the first quadrant, i.e., $[0,1.1]^{2}$. We mesh the domain using a structured triangular grid by subdividing each cell of a uniform $90 \times 90$ quadrilateral mesh into four triangles of equal size. In this test, the initial density is given by the uniform value $\rho_{0}=1.0$, the initial velocity is zero and the initial internal energy is a numerical approximation to $e_{0} \delta(\boldsymbol{x})$, where $e_{0}=0.9792$ designates the strength of the blast wave and $\delta(\boldsymbol{x})$ is the Dirac delta function centered at the origin. A cold background environment is specified by the small internal energy value $1 e-14$.

At the terminal time of the simulation, $T=1.0$, the front of the blast wave has not reached the domain boundary, thus we specify strong velocity boundary condition $\boldsymbol{v} \cdot \boldsymbol{n}=0$ on all boundaries. To assess the numerical stability of the proposed method (in the sense of bounded, non-oscillatory solutions) in various computational settings, we consider (a) the Eulerian computation, (b) the ALE computation with Laplacian smoothing, and (c) the pure Lagrangian computation. With each choice, we further test the time-averaged (T-ave.) convection velocity and the time-interpolated (T-interp.) convection velocity.

We plot the numerical pressure at $T=1.0$ in Figure 7.18 as well as the final mesh locations. In all situations, we obtain stable solutions. The accuracy of the solution is largely dependent on the local mesh resolution near the shock fronts; thus the Lagrangian computations lead to the sharpest shock fronts among all, the ALE computations are a little more dissipative than the Lagrangian ones, whereas the Eulerian computations smear the shock fronts the most. Comparing the time-interpolated strategy and the time-average strategy to compute the convection velocity $\boldsymbol{v}-\hat{\boldsymbol{v}}$, they are almost indistinguishable in the figures: A more detailed analysis shows the time-interpolated approach is slightly more dissipative than the time-averaged approach.

A quantitative comparison against the analytical exact solution along the diagonal direction in the $x-y$ plane is provided in Figure 7.19. It is visible that the computed ALE solutions have nearly the same accuracy as the Lagrangian solutions, and are both more accurate (less dissipative) than the Eulerian solutions. A closer examination of the results shows that the time-averaged convective velocity (solid curves) provides slightly more accurate results than the time-interpolated one (dashed curves). Aside from this detail, the numerical solution converges to the analytical in Figure 7.20, for density and internal energy. This figure shows the global views (left) and zoomed views (right) of both variables. The pressure and velocity converge in a similar way to the density and they are not plotted.

At the end of this test, we demonstrate the symmetry-preserving property of the method by computing the solutions on an anisotropic grid. Particularly we subdivide each cell of a uniform $180 \times 90$ quadrilateral mesh into four triangles of equal size. The final numerical pressure at $T=1.0$ as well as the final mesh locations are plotted in Figure 7.21. We only present the "T-ave" solutions here as the other strategy leads to similar results. All the computations capture the features of the Sedov blast very well. We also note that the peak value of the pressure differs slightly in the two axial directions. This difference is only noticeable in the Eulerian case because the mesh resolution does not improve as the blast wave propagates, and is a byproduct of the different resolutions of the grid along the vertical and horizontal 


\section{Pressure}

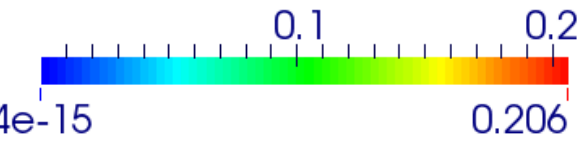

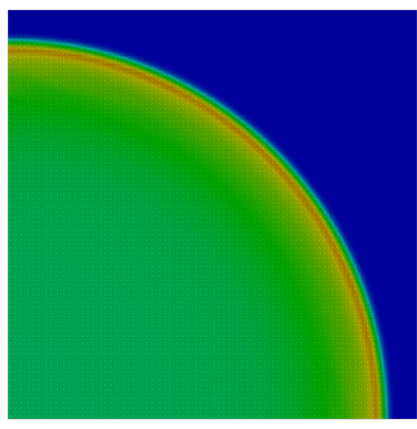

(a) Eulerian, T-ave.

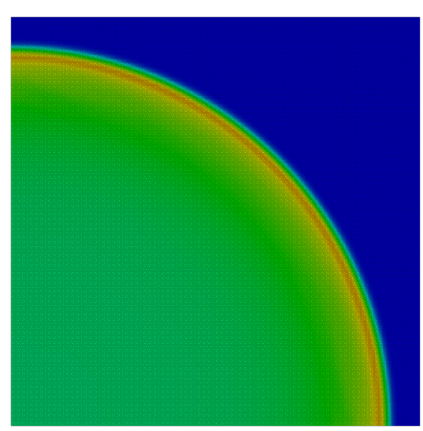

(d) Eulerian, T-interp.

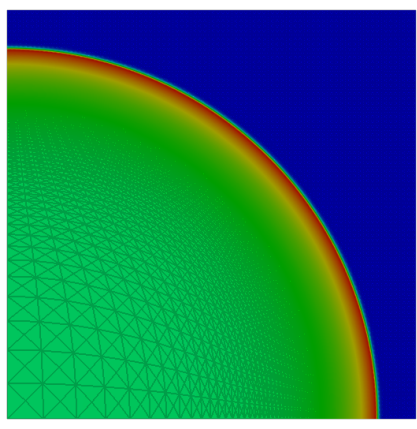

(b) ALE, T-ave.

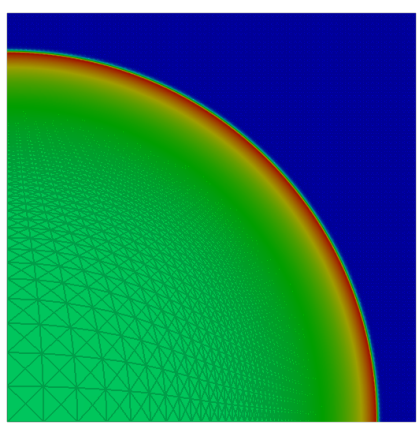

(e) ALE, T-interp.

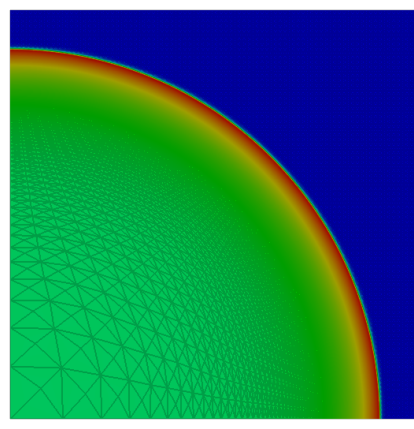

(c) Lagrangian, T-ave.

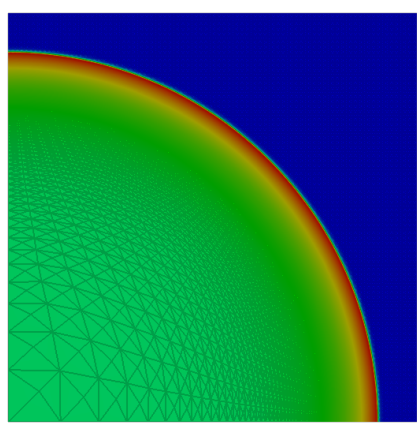

(f) Lagrangian, T-interp.

Figure 7.18: Pressure at $T=1.0$ for: Eulerian (left), ALE (middle), and Lagrangian (right) computations; and time-averaged convection velocity (upper) and time-interpolated convection velocity (lower). All numerical solutions computed on a $4 \times 90 \times 90$ grid, and use VMS-stabilization with $c_{\tau}=2.5$ and entropy viscosity $c_{\text {ent }}=40.0$.
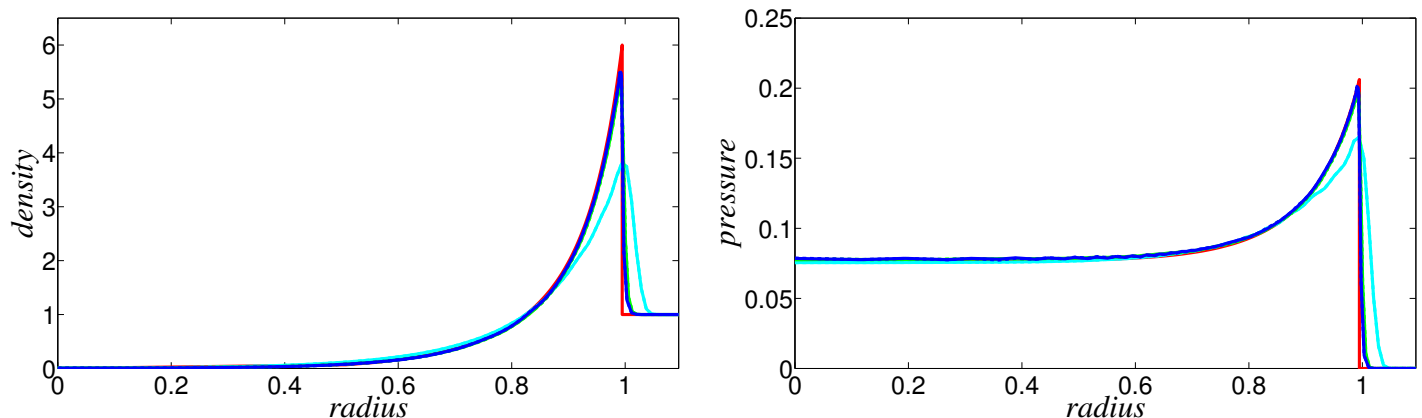

Figure 7.19: Analytical solutions (red) vs. numerical solutions (other colors) along the $x$ - $y$ diagonal direction. The solid lines and dashed lines represent the time-averaged and the time-interpolated mesh convective velocities, respectively. The cyan, green, and blue curves are computed using the Eulerian, ALE, and Lagrangian methods, respectively. 

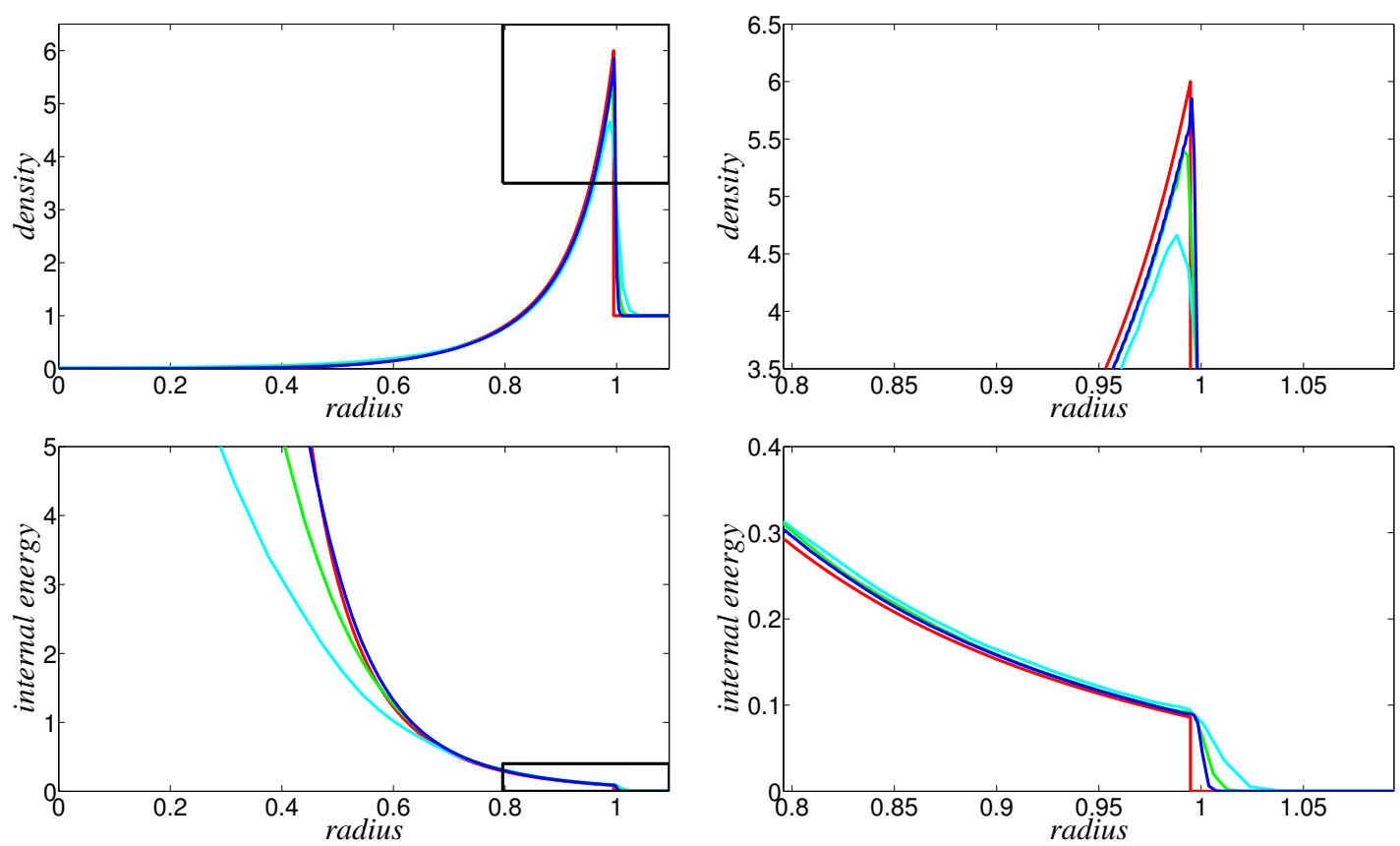

Figure 7.20: Convergence of the numerical solution (ALE and time-averaged mesh convective velocity) to the analytical one along the $x-y$ diagonal direction. The analytical solution is plot in red, whereas the numerical ones computed on the $45 \times 45,90 \times 90$, and $180 \times 180$ grids are plotted in cyan, green, and blue, respectively.

\section{Pressure}
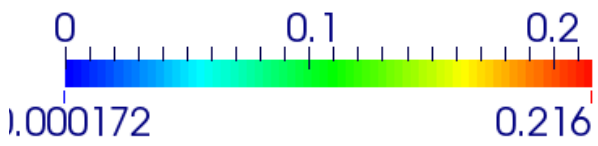

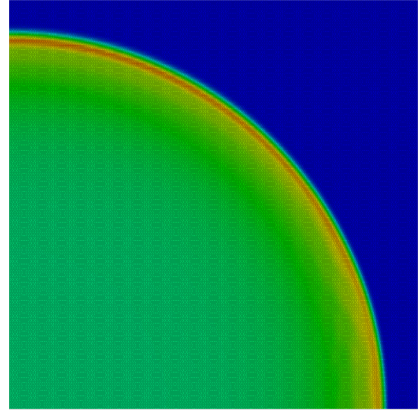

(a) Eulerian, T-ave.

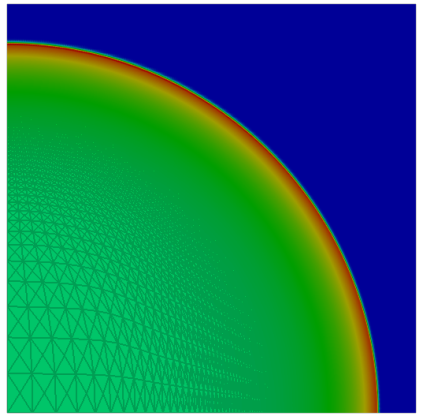

(b) ALE, T-ave.

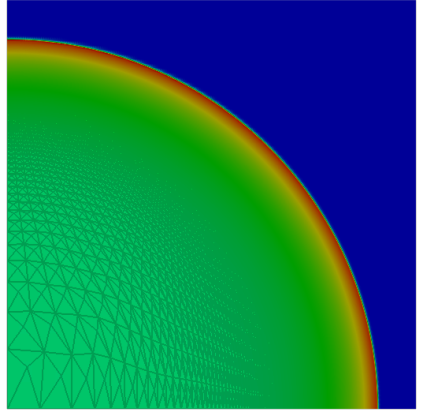

(c) Lagrangian, T-ave.

Figure 7.21: Pressure at $T=1.0$ for: Eulerian (left), ALE (middle), and Lagrangian (right) computations; and time-averaged convection velocity (upper) and time-interpolated convection velocity (lower). All numerical solutions computed on a $4 \times 180 \times 90$ grid, and use VMS-stabilization with $c_{\tau}=2.5$ and entropy viscosity $c_{\text {ent }}=40.0$. 


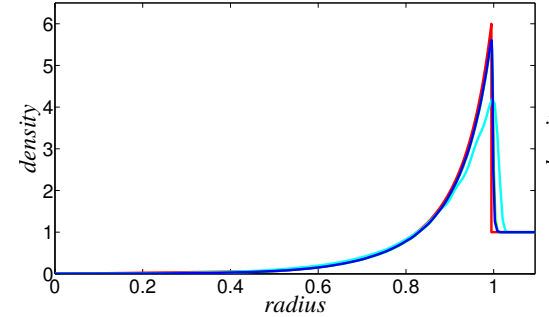

(a) $x$-direction.

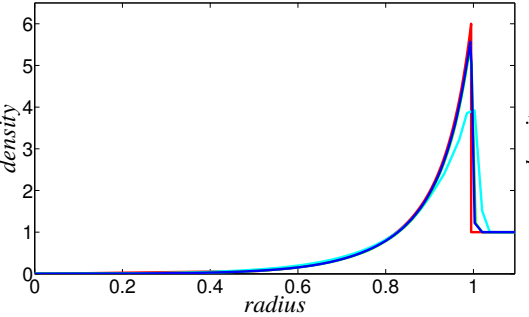

(b) Diagonal direction.

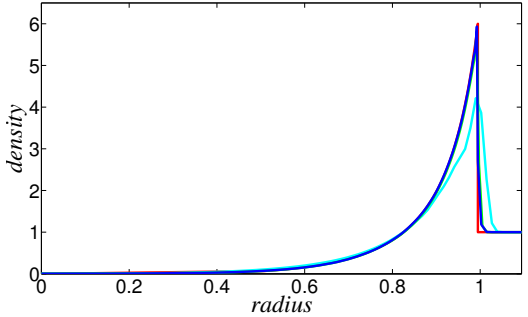

(c) $y$-direction.

Figure 7.22: Analytical solutions (red) vs. numerical solutions (other colors) along directions on an anisotropic $4 \times 180 \times 90$ grid. The cyan, green, and blue curves are computed using the Eulerian, ALE, and Lagrangian methods, respectively. Only the time-averaged mesh convective velocities are considered.

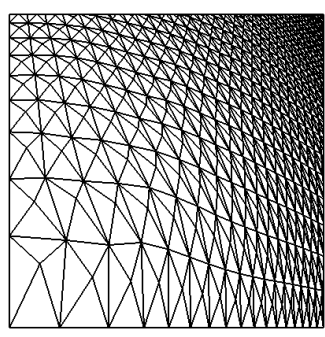

(a) $h_{e}, t=1.0 ;$ lower $[0,0.55]^{2}$.

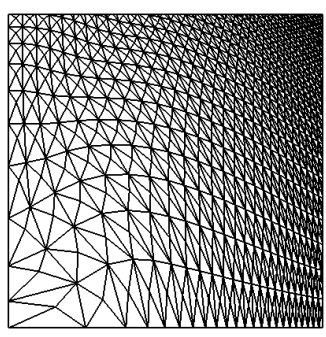

(b) $h_{e}^{1}, t=1.0$; lower $[0,0.55]^{2}$.

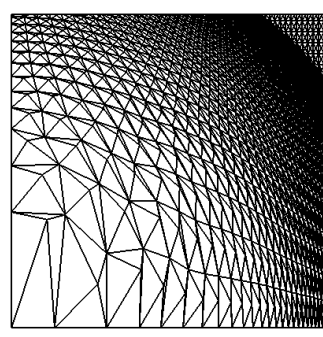

(c) $h_{e}^{2}, t=0.2$; lower $[0,0.35]^{2}$.

Figure 7.23: Mesh positions by using various element length estimates in the VMS-stabilization and the entropy viscosity. For the choices $h_{e}$ and $h_{e}^{1}$ (left and middle), the mesh positions in the region $[0,0.55]^{2}$ at $t=1.0$ are plotted; whereas for the choice $h_{e}^{2}$ (right), the mesh position in the region $[0,0.35]^{2}$ at $t=0.2$. All computations are Lagrangian, use time-averaged mesh convective velocity, and parameters $c_{\tau}=2.5$ and $c_{\mathrm{ent}}=40.0$.

directions. This phenomenon is more readily revealed by plotting the densities along three directions, as shown in Figure 7.22

Finally, we use this test to compare two alternatives to 3.28 when computing the mesh size $h_{e}$ in the VMSstabilization and the artificial viscosity. The first method is to use the largest edge length of an element:

$$
h_{e}^{1}=\max _{\boldsymbol{x}_{A}, \boldsymbol{x}_{B} \in \overline{\Omega_{e}} ; A \neq B}\left\|\boldsymbol{x}_{A}-\boldsymbol{x}_{B}\right\|
$$

where $\boldsymbol{x}_{A}$ and $\boldsymbol{x}_{B}$ are distinct nodes of the element $\Omega_{e}$. The second choice is to use the diameter of the largest circle or sphere inscribed into the element $\Omega_{e}$, as already done in Section 6.3 when computing the stable time step, Namely

$$
h_{e}^{2}=h_{e}^{\mathrm{cfl}} .
$$

These two choices generally tend to overestimate and underestimate the element size, respectively, especially for high aspect ratio elements. For example, with everything else held the same, we apply $h_{e}^{1}$ and $h_{e}^{2}$ to perform the Lagrangian computation with time-averaged mesh convective velocity. The mesh positions are plotted in Figure 7.23. Comparing the final mesh positions, $h_{e}^{1}$ leads to a more mesh dependent solutions than $h_{e}$ : In Figure 7.23b, we see that elements deform more in the direction where the grid is more refined. This is expected, because $h_{e}^{1}$ is highly sensitive to the element orientation. Using the choice $h_{e}^{2}$, instead, highly squeezed elements are produced and the simulation halts around $t=0.2$ (Figure $7.23 \mathrm{c}$ ).

We emphasize, however, that we did not optimized the viscosity parameter for these choices of mesh size. Nevertheless, our experience indicates that $h_{e}$ defined as in 3.28 is a robust choice for a wide range of applications, whereas $h_{e}^{1}$ and $h_{e}^{2}$ tend to lead to more mesh-dependent solutions or squeezed elements. 


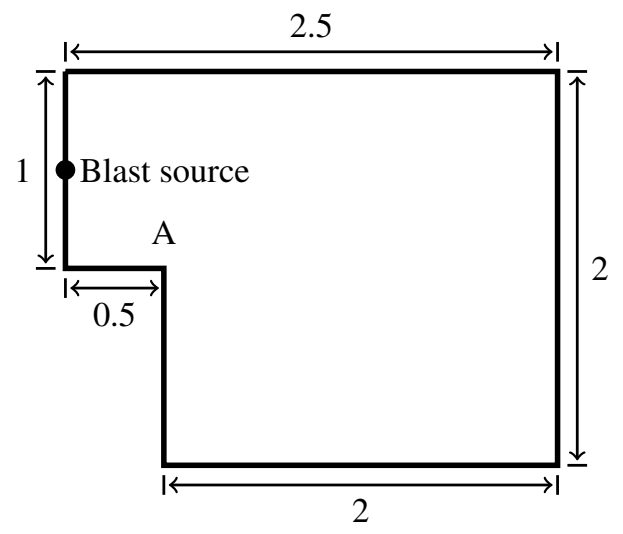

Figure 7.24: The setup of the blast wave past a backward-facing step.

\subsubsection{Blast wave past a backward-facing step (Eulerian)}

The blast wave past a backward-facing step is a very challenging problem in shock hydrodynamics, because the flow exhibits strong shocks, vorticity, and geometric singularities at the same time. The Lagrangian/remap computation requires special attention to handle the data transfer of linear momentum or flow velocity [15] in order to solve this problem correctly. Figure 7.24 describes the problem setup: A Sedov blast wave of strength $e_{0}=12.57$ starts at the center of the left-most boundary that has unit length and this boundary is modeled as a symmetry plane. Then it generates a right-moving shock front through the short channel ahead of the backward-facing step that locates the distance of 0.5 downstream the left boundary. This shock front will reach the corner (point A) of the step at about $t=0.15$, where the flow velocity is zero, and is forced to rotate in a clockwise direction. The upper edge of the computational domain is a slip wall, and the domain to the lower-right of the corner is sufficient large to prevent the interaction of the shock fronts with the lower and the right boundaries until the termination time of simulation: $T=0.8$.

We use an Eulerian unstructured triangular mesh with 262,446 elements and 131,944 nodes. The flow patterns are illustrated by the pressure history in Figure 7.25, where both the time-interpolated convection velocity strategy (right column) and the time-averaged convection velocity strategy (left column) are considered. The time-averaged strategy and the time-interpolated strategy lead to almost identical results.

Finally, to demonstrate the effectiveness of the entropy viscosity for shock hydrodynamics, we plot the intensity of the entropy residual for the previous computations in Figure 7.26 These plots are arranged in the same order as Figure 7.25, and we can clearly see the localization of the entropy viscosity near the shock fronts.

\subsubsection{Conclusions}

The numerical solutions obtained with our method compare well with classical Lagrangian schemes. One important reason is that the artificial mass flux vanishes in the Lagrangian limit, when the mesh convective velocity is zero, and, as a consequence, no artificial mass transfer occurs between neighboring nodes. The ALE solutions compare extremely well with the Lagrangian ones, with an important practical advantage: Because the time step size usually scales with the shortest element size in the mesh (near shock fronts), the ALE computations allow much larger time step sizes than the Lagrangian ones. Hence, ALE simulations can enhance computational efficiency without losing much of the mesh resolution near the shocks.

\subsection{Three-dimensional example - quintuple point problem (Eulerian, ALE with Laplacian smoothing)}

To conclude, we present a three-dimensional example, a generalization of the widely known triple point problem in two space dimensions [12, 15, 16]. The computational domain is the box $\Omega=[0,7] \times[0,3] \times[0,3]$, and it is separated in five regions as shown in Figure 7.27. In particular, region I starts from the front end of the domain and terminates at the distance 1.0, and the remaining sub-region is divided into four smaller boxes of equal sizes. The fluid is ideal 

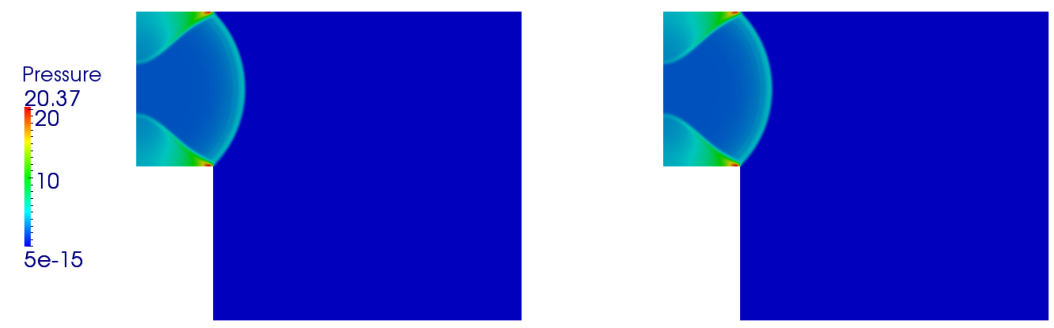

(a) $t=0.15$. T-averaged vs. T-interpolated.
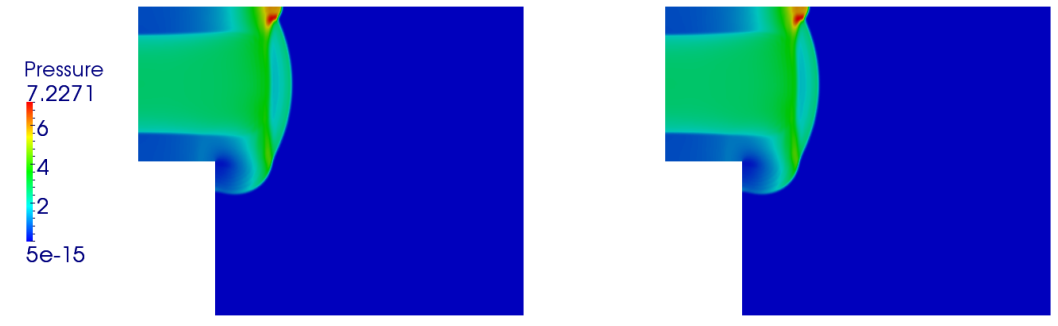

(b) $t=0.3$. T-averaged vs. T-interpolated.
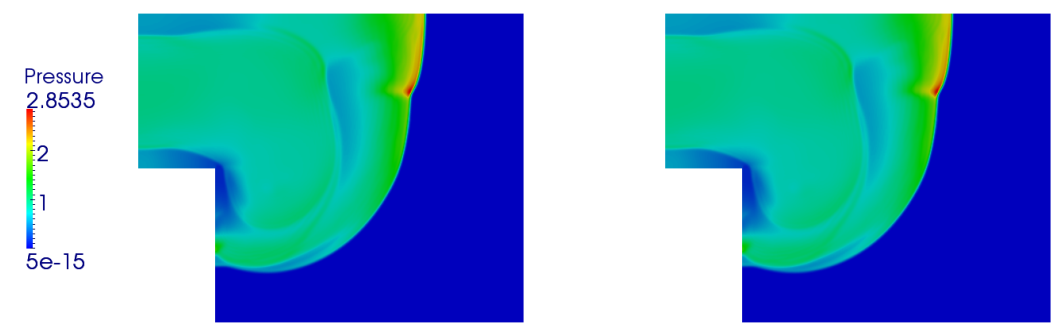

(c) $t=0.8$. T-averaged vs. T-interpolated.

Figure 7.25: Pressure for the Sedov blast wave past a backward-facing step: at $t=0.15$ (top), $t=0.3$ (middle), and $t=0.8$ (bottom) and timeaveraged convection velocity (left) and time-interpolated convection velocity (right). All numerical solutions use VMS-stabilization with $c_{\tau}=2.5$ and entropy viscosity $c_{\text {ent }}=40.0$. 

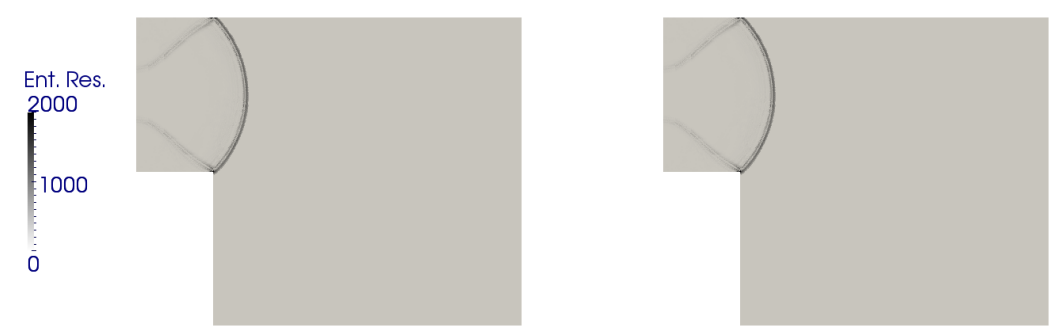

(a) $t=0.15$. T-averaged vs. T-interpolated.
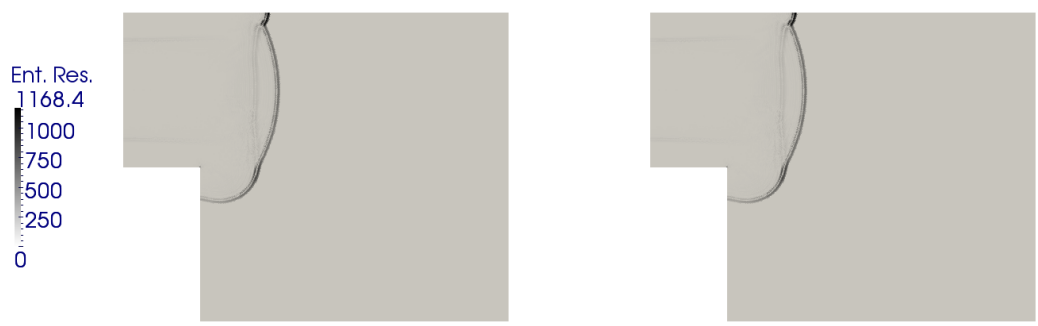

(b) $t=0.3$. T-averaged vs. T-interpolated.

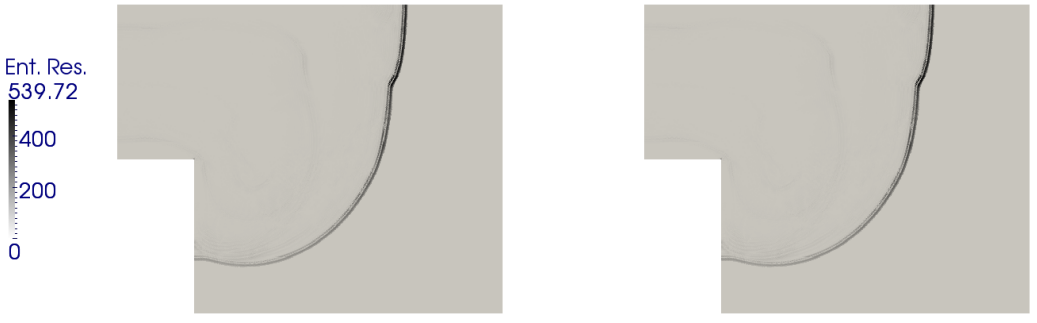

(c) $t=0.8$. T-averaged vs. T-interpolated.

Figure 7.26: Absolute value of the entropy residual for the Sedov blast wave past a backward-facing step: at $t=0.15$ (top), $t=0.3$ (middle), and $t=0.8$ (bottom) and time-averaged convection velocity (left) and time-interpolated convection velocity (right). All numerical solutions use VMS-stabilization with $c_{\tau}=2.5$ and entropy viscosity $c_{\text {ent }}=40.0$. The peak entropy residual at $t=0.15$ occurs at the corner of the step and takes the value $\sim 8.8 e+4$. For the purpose of visualization, we cropped the range of the residual to 2,000 .

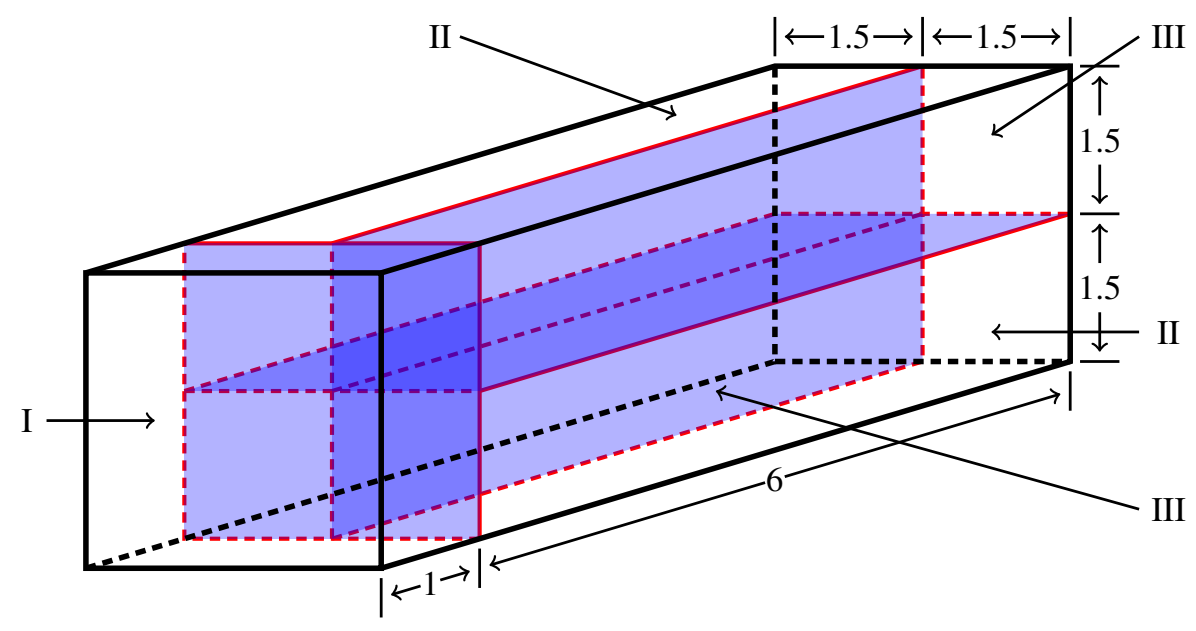

Figure 7.27: The quintuple point problem setup. 


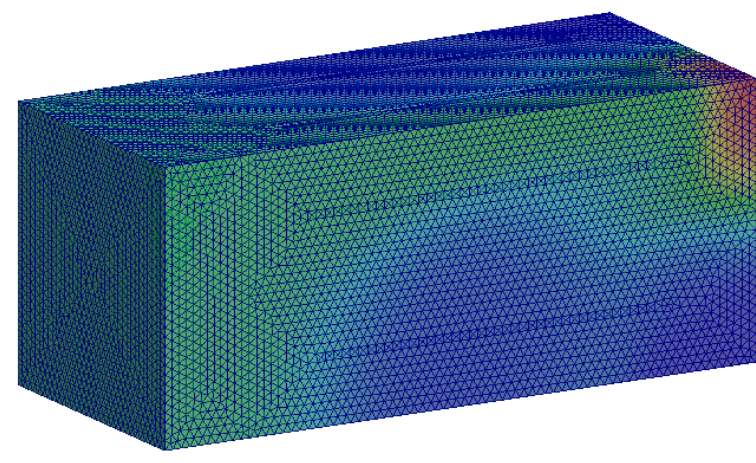

(a) Eulerian mesh at $T=5.0$.

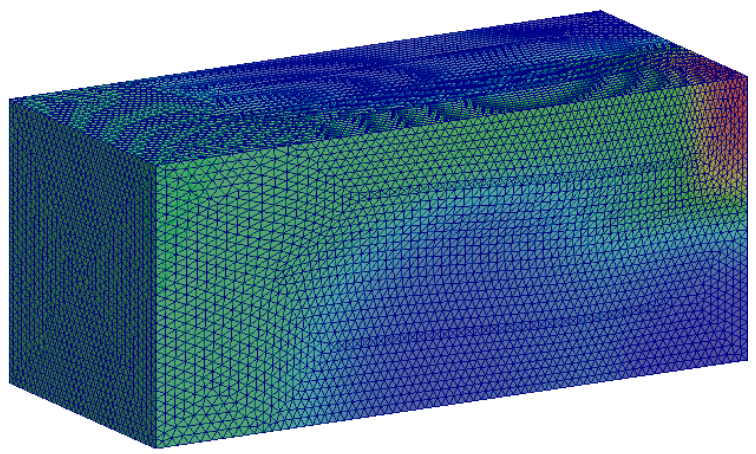

(b) ALE mesh at $T=5.0$.

Figure 7.28: The location of a fully unstructured grid with 512,909 tetrahedra and 91,221 nodes. The grids are plotted on top of internal energy contours in the range $[0.233,3.35]$ at $T=5.0$ (cf. Figure 7.30 .

gas with $\gamma=1.4$, and the initial flow conditions are

$$
\left\{\begin{array}{l}
\rho_{\mathrm{I}}=1.0 \\
\boldsymbol{u}_{\mathrm{I}}=\mathbf{0 . 0} \\
p_{\mathrm{I}}=1.0
\end{array}, \quad\left\{\begin{array}{l}
\rho_{\mathrm{II}}=1.0 \\
\boldsymbol{u}_{\mathrm{II}}=\mathbf{0 . 0} \\
p_{\mathrm{II}}=0.1
\end{array}, \quad\left\{\begin{array}{l}
\rho_{\mathrm{III}}=0.125 \\
\boldsymbol{u}_{\mathrm{III}}=\mathbf{0 . 0} \\
p_{\mathrm{III}}=0.1
\end{array},\right.\right.\right.
$$

here the region numbers are designated in Figure 7.27. Note that each of the two diagonal pairs of the four boxes on the back shares the same initial data.

We set all the boundaries to be slip walls (i.e., homogeneous Dirichlet condition on the normal velocity). An fully unstructured tetrahedral grid with 512,909 elements and 91,221 nodes is used to mesh the domain, and we solve the problem until $T=5.0$ using the same stabilization, artificial viscosities, and Courant number as before. Both an Eulerian computation and an ALE computation are performed, and the final mesh positions are illustrated in Figure 7.28a and Figure 7.28b, respectively. In both cases, we use the time-averaged strategy to compute the convection velocity. The evolutions of (the contours of) the density, internal energy, pressure, and flow Mach number are plotted in Figure 7.297 .32

This example demonstrates the three-dimensional capability of the proposed method. In addition, the problem is an extension of the Riemann problem in 1D and the computational domain is fixed in time. Classical numerical methods for solving these problems are in general Eulerian, and the mesh remains fixed throughout the computation. By introducing a mesh motion and smoothing processes, we are able to perform an ALE computation on the fixed domain, and obtain sharper shock fronts with respect to Eulerian computations.

\section{Conclusions}

We presented a monolithic ALE method for highly transient shock hydrodynamics flows, using linear finite elements on unstructured grids. Linear and nonlinear numerical stabilization mechanisms are provided in the form of VMS operators and entropy viscosity discontinuity capturing operators, respectively. Our approach allows for a reduction in computational effort and a simplification in the formulation of inflow and outflow boundary conditions with respect to traditional shock hydrodynamics algorithms (hydrocodes), which typically adopt a multistage Lagrangian+remap strategy.

Another aspect that we believe important in our work is related to the fact that, to date, practically no literature has been published on the use of monolithic stabilized or VMS finite element methods for Eulerian or ALE unsteady computations of strong shocks. With our work, we aimed at closing this gap. 

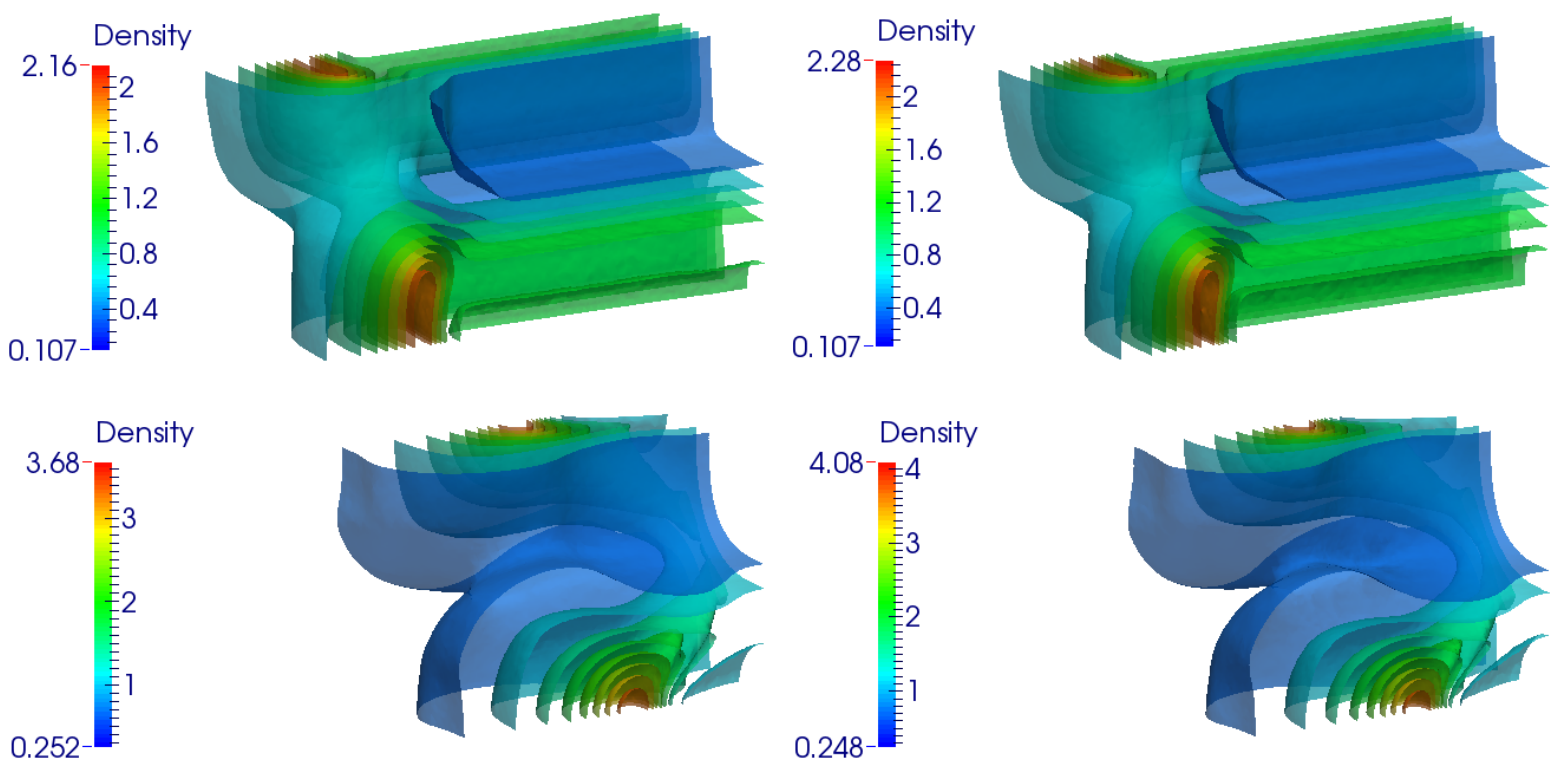

(a) Eulerian T-ave., density.

(b) ALE T-ave., density.

Figure 7.29: Quintuple point problem. Evolution of density contours at $t=1.5$ (top) and $t=5.0$ (bottom), using Eulerian (left) and ALE (right) methods. All numerical solutions use VMS-stabilization with $c_{\tau}=2.5$ and entropy viscosity $c_{\text {ent }}=40.0$.
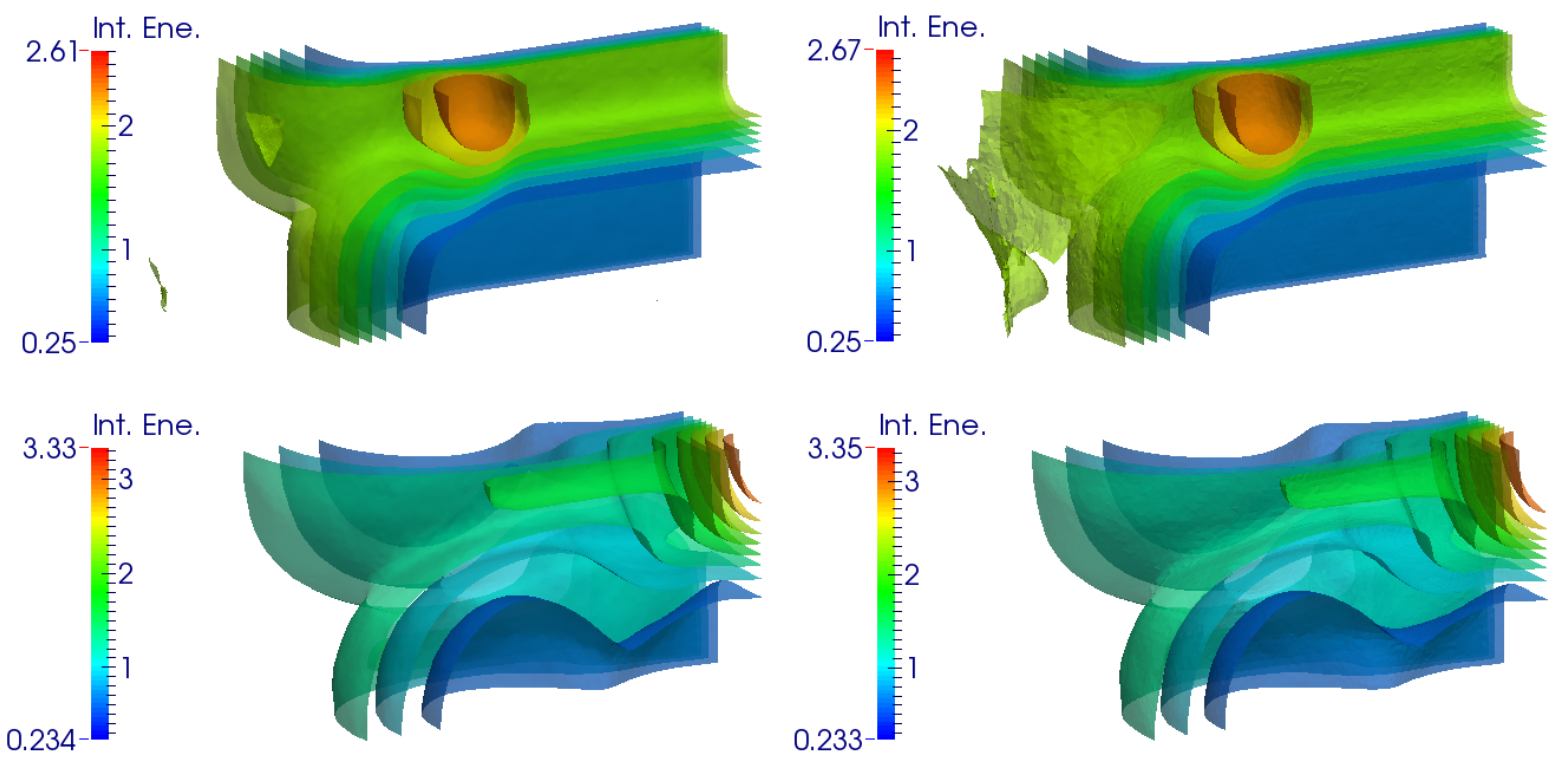

(a) Eulerian T-ave., internal energy.

(b) ALE T-ave., internal energy.

Figure 7.30: Quintuple point problem. Evolution of internal energy contours at $t=1.5$ (top) and $t=5.0$ (bottom), using Eulerian (left) and ALE (right) methods. All numerical solutions use VMS-stabilization with $c_{\tau}=2.5$ and entropy viscosity $c_{\text {ent }}=40.0$. 

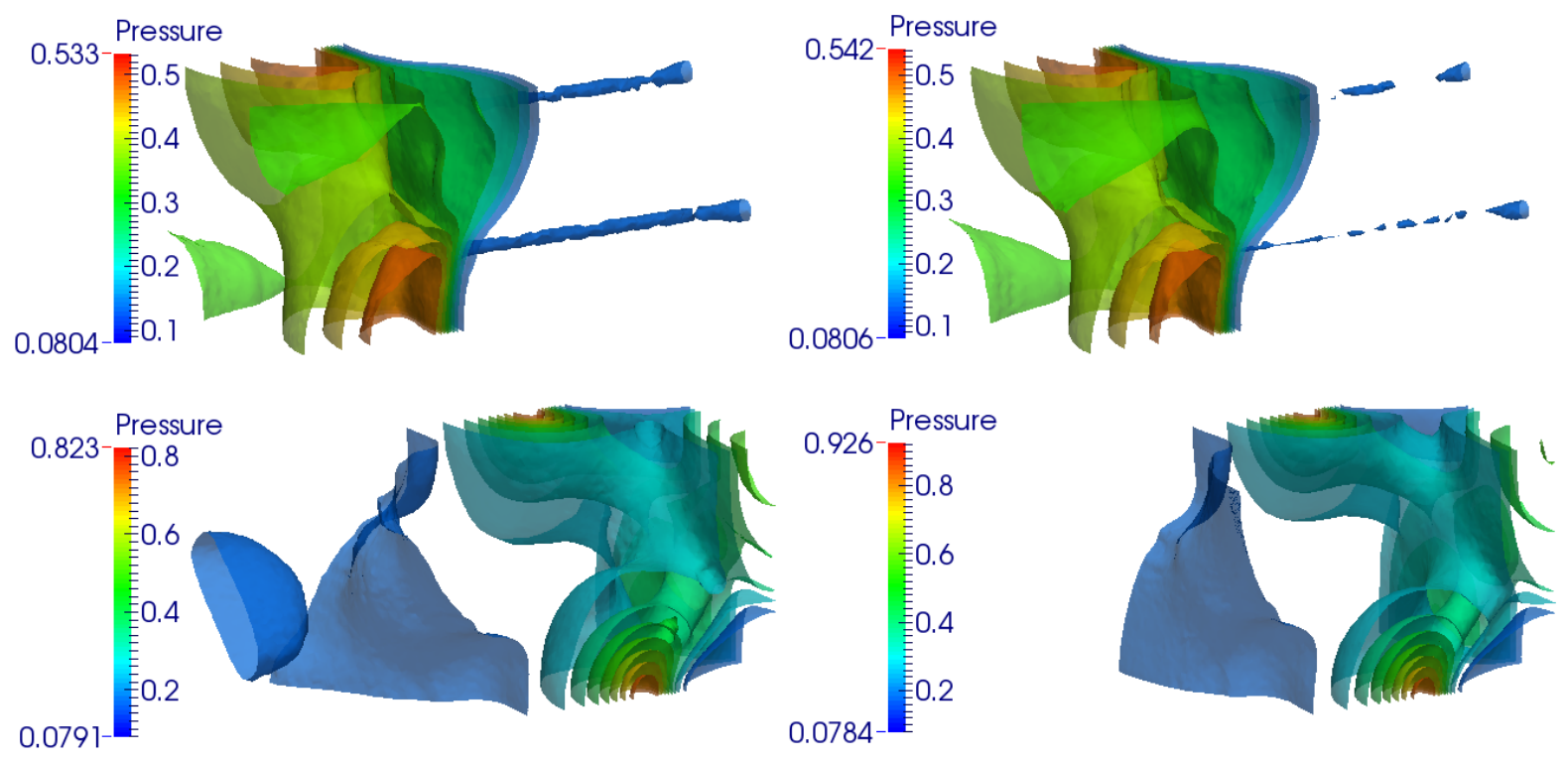

(a) Eulerian T-ave., pressure.

(b) ALE T-ave., pressure.

Figure 7.31: Quintuple point problem. Evolution of pressure contours at $t=1.5$ (top) and $t=5.0$ (bottom), using Eulerian (left) and ALE (right) methods. All numerical solutions use VMS-stabilization with $c_{\tau}=2.5$ and entropy viscosity $c_{\text {ent }}=40.0$.
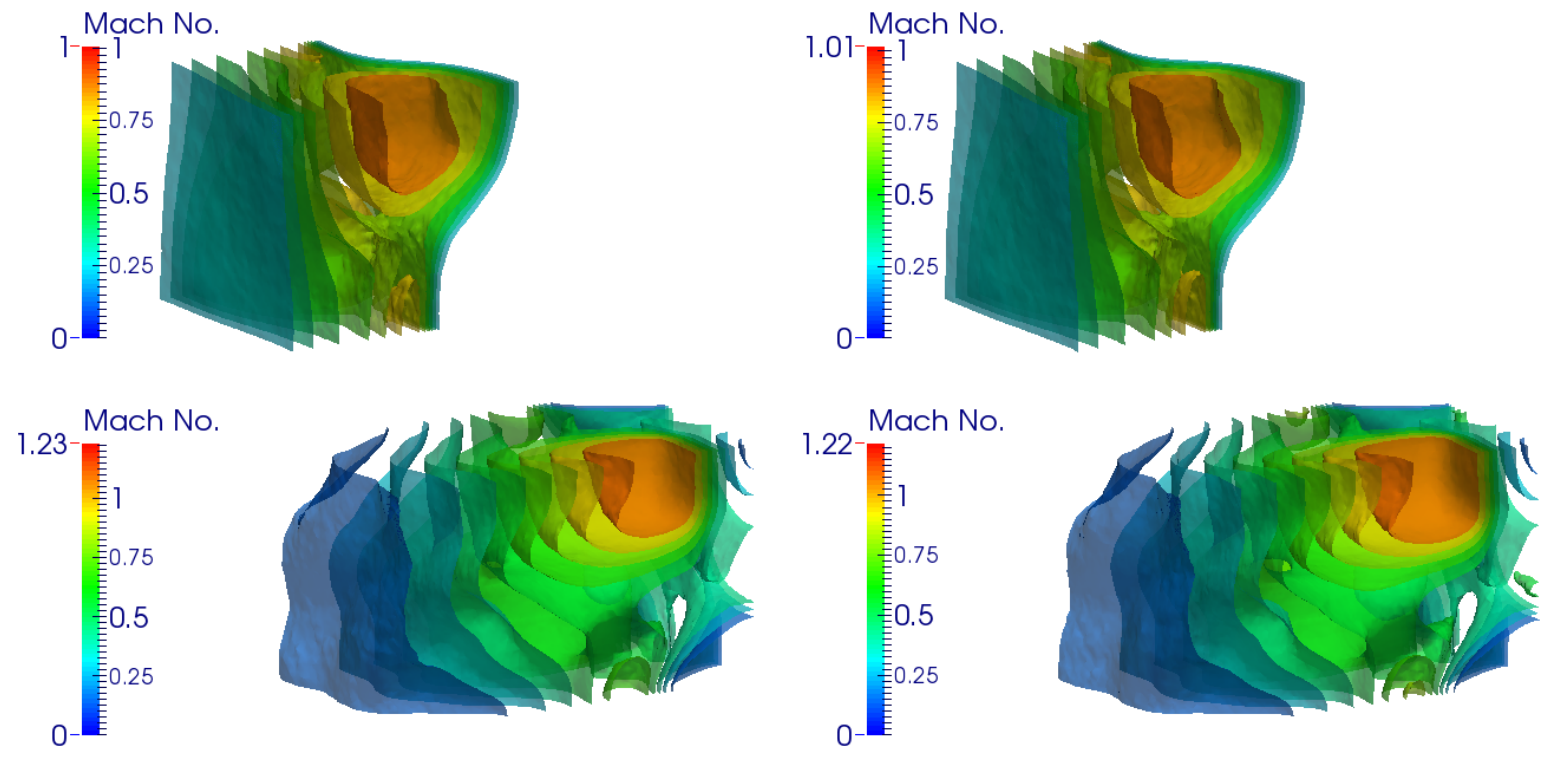

(a) Eulerian T-ave., Mach number.

(b) ALE T-ave., Mach number.

Figure 7.32: Quintuple point problem. Evolution of Mach number contours at $t=1.5$ (top) and $t=5.0$ (bottom), using Eulerian (left) and ALE (right) methods. All numerical solutions use VMS-stabilization with $c_{\tau}=2.5$ and entropy viscosity $c_{\text {ent }}=40.0$. 


\section{Acknowledgement}

This work was supported by the Sandia National Laboratories through the Laboratory Directed Research \& Development program. Sandia National Laboratories is a multi-program laboratory operated by Sandia Corporation, a wholly owned subsidiary of Lockheed Martin company, for the U.S. Department of Energy's National Nuclear Security Administration under contract DE-AC04-94AL85000. The authors would like to thank Dr. John Shadid and Dr. Eric Cyr at Sandia National Laboratories for very fruitful discussions.

\section{References}

[1] D. J. Benson, An efficient, accurate, simple ALE method for nonlinear finite element programs, Comput. Methods Appl. Mech. Eng. 72 (1989) 305-350.

[2] D. J. Benson, Computational methods in Lagrangian and Eulerian hydrocodes, Comput. Methods Appl. Mech. Eng. 99 (1992) $235-394$.

[3] E. J. Caramana, M. J. Shashkov, P. P. Whalen, Formulations of artificial viscosity for multi-dimensional shock wave computations, J. Comput. Phys. 144 (1998) 70-97.

[4] G. Scovazzi, M. A. Christon, T. J. R. Hughes, J. N. Shadid, Stabilized shock hydrodynamics: I. A Lagrangian method, Comput. Methods Appl. Mech. Eng. 196 (2007) 923-966.

[5] G. Scovazzi, Stabilized shock hydrodynamics: II. Design and physical interpretation of the SUPG operator for Lagrangian computations, Comput. Methods Appl. Mech. Eng. 196 (2007) 966-978.

[6] G. Scovazzi, E. Love, M. J. Shashkov, Multi-scale Lagrangian shock hydrodynamics on Q1/P0 finite elements: Theoretical framework and two-dimensional computations, Comput. Methods Appl. Mech. Eng. 197 (2008) 1056-1079.

[7] P.-H. Maire, A high-order cell-centered Lagrangian scheme for two-dimensional compressible fluid flows on unstructured meshes, J. Comput. Phys. 228 (2009) 2391-2425.

[8] G. Scovazzi, J. N. Shadid, E. Love, W. J. Rider, A conservative nodal variational multiscale method for Lagrangian shock hydrodynamics, Comput. Methods Appl. Mech. Eng. 199 (2010) 3059-3100.

[9] A. L. Ortega, G. Scovazzi, A geometrically-conservative, synchronized, flux-corrected remap for arbitrary Lagrangian-Eulerian computations with nodal finite elements, J. Comput. Phys. 230 (2011) 6709-6741.

[10] G. Scovazzi, A. L. Ortega, Algebraic flux correction and geometric conservation in arbitrary Lagrangian-Eulerian computations, in: L. D. Kuzmin D., T. S. (Eds.), Flux-Corrected Transport: Principles, Algorithms, and Applications, Springer-Verlag, Berlin-New York, second edition, 2004

[11] G. Scovazzi, Lagrangian shock hydrodynamics on tetrahedral meshes: A stable and accurate variational multiscale approach, J. Comput. Phys. 231 (2012) 8029-8069.

[12] V. A. Dobrev, T. V. Kolev, R. N. Rieben, High-order curvilinear finite element methods for Lagrangian hydrodynamics, SIAM J. Sci. Comput. 34 (2012) B606-B641.

[13] G. Luttwak, J. Falcovitz, VIP (vector image polygon) multi-dimensional slope limiters for scalar variables, Comput. Fluids 83 (2013) 90-97.

[14] M. Kucharik, M. Shashkov, Conservative multi-material remap for staggered multi-material arbitrary Lagrangian-Eulerian methods, J. Comput. Phys. 258 (2014) 268-304.

[15] X. Zeng, G. Scovazzi, A frame-invariant vector limiter for flux corrected nodal remap in arbitrary Lagrangian-Eulerian flow computations, J. Comput. Phys. 270 (2014) 753-783.

[16] R. Loubére, P.-H. Maire, M. Shashkov, J. Breil, S. Galera, ReALE: A reconnection-based arbitrary-Lagrangian-Eulerian method, J. Comput. Phys. 229 (2010) 4724-4761.

[17] J. Velechovský, M. Kuchař́k, R. Liska, M. Shashkov, P. Váchal, Symmetry- and essentially-bound-preserving flux-corrected remapping of momentum in staggered ALE hydrodynamics, J. Comput. Phys. 255 (2013) 590-611.

[18] J.-L. Guermond, R. Pasquetti, Entropy-based nonlinear viscosity for Fourier approximations of conservation laws, C. R. Math. 346 (2008) $801-806$

[19] J. L. Guermond, R. Pasquetti, B. Popov, Entropy viscosity for conservation laws, in: J. C. F. Pereira, A. Sequeira (Eds.), European Conference on Computational Fluid Dynamics, Lisbon, Portugal. ECCOMAS CFD 2010.

[20] J.-L. Guermond, R. Pasquetti, B. Popov, Entropy viscosity method for nonlinear conservation laws, J. Comput. Phys. 230 (2011) $4248-4267$.

[21] J.-L. Guermond, R. Pasquetti, B. Popov, From suitable weak solutions to entropy viscosity, J. Sci. Comput. 49 (2011) 35-50.

[22] J.-L. Guermond, B. Popov, V. Tomov, Entropyviscosity method for the single material euler equations in lagrangian frame, Comput. Methods Appl. Mech. Eng. 300 (2016) 402-426.

[23] E. Love, G. Scovazzi, On the angular momentum conservation and incremental objectivity properties of a predictor/multi-corrector method for Lagrangian shock hydrodynamics, Comput. Methods Appl. Mech. Eng. 198 (2009) 3207-3213.

[24] J.-L. Guermond, B. Popov, Viscous regularization of the Euler equations and entropy principles, SIAM J. Appl. Math. 74 (2014) $284-305$.

[25] Árpád Csík, M. Richhiuto, H. Deconinck, A conservative formulation of the multidimensional upwind residual distribution schemes for general nonlinear conservation laws, J. Comput. Phys. 179 (2002) 286-312.

[26] M. Ricchiuto, Árpád Csík, H. Deconinck, Residual distribution for general time-dependent conservation laws, J. Comput. Phys. 209 (2005) 249-289.

[27] H. Deconinck, M. Ricchiuto, Residual distribution schemes: Foundations and analysis, in: E. Stein, R. de Borst, T. J. R. Hughes (Eds.), Encyclopedia of Computational Mechanics, John Wiley \& Sons, 2004.

[28] M. Ricchiuto, A. Bollermann, Stabilized residual distribution for shallow water simulations, J. Comput. Phys. 228 (2009) $1071-1115$.

[29] R. Abgrall, A. Larat, M. Ricchiuto, Construction of very high order residual distribution schemes for steady inviscid flow problems on hybrid unstructured meshes, J. Comput. Phys. 230 (2011) 4103-4136. 
[30] L. Arpaia, M. Ricchiuto, R. Abgrall, An ALE formulation for explicit Runge-Kutta residual distribution, J. Sci. Comput. 63 (2015) $502-547$.

[31] J. Waltz, N. R. Morgan, T. R. Canfield, M. R. J. Charest, L. D. Risinger, J. G. Wohlbier, A three-dimensional finite element arbitrary Lagrangian-Eulerian method for shock hydrodynamics on unstructured grids, Comput. Fluids 92 (2014) $172-187$.

[32] J. Donea, S. Giuliani, J. P. Halleux, An arbitrary Lagrangian-Eulerian finite element method for transient dynamic fluid-structure interactions, Comput. Methods Appl. Mech. Eng. 33 (1982) 689-723.

[33] S. Pipernoa, C. Farhat, B. Larrouturou, Partitioned procedures for the transient solution of coupled aroelastic problems Part I: Model problem, theory and two-dimensional application, Comput. Methods Appl. Mech. Eng. 124 (1995) 79-112.

[34] P. Geuzaine, C. Grandmont, C. Farhat, Design and analysis of ALE schemes with provable second-order time-accuracy for inviscid and viscous flow simulations, J. Comput. Phys. 191 (2003) 206-227.

[35] C. Farhat, P. Geuzaine, G. Brown, Application of a three-field nonlinear fluidstructure formulation to the prediction of the aeroelastic parameters of an F-16 fighter, Comput. Fluids 32 (2003) 3-29.

[36] K. Takizawa, T. E. Tezduyar, Multiscale space-time fluid-structure interaction techniques, Comput. Mech. 48 (2011) 247-267.

[37] T. J. R. Hughes, G. Scovazzi, T. E. Tezduyar, Stabilized methods for compressible flows, J. Sci. Comput. 43 (2007) $343-368$.

[38] G. Scovazzi, A discourse on Galilean invariance and SUPG-type stabilization, Comput. Methods Appl. Mech. Eng. 196 (2007) $1108-1132$.

[39] G. Scovazzi, E. Love, A generalized view on Galilean invariance in stabilized compressible flow computations, Int. J. Numer. Meth. Fl. 64 (2010) 1065-1083.

[40] G. Scovazzi, Galilean invariance and stabilized methods for compressible flows, Int. J. Numer. Meth. Fl. 54 (2007) $757-778$.

[41] C. J. Chapman, High Speed Flow, volume 23 of Cambridge Texts in Applied Mathematics, Cambridge University Press, 2000.

[42] J.-M. Ghidaglia, F. Pascal, On boundary conditions for multidimensional hyperbolic systems of conservation laws in the finite volume framework, 2002. Technical report, CMLA, ENS de Cachan.

[43] T. J. R. Hughes, Multiscale phenomena: Green's functions, the Dirichlet-to-Neumann formulation, subgrid scale models, bubbles and the origins of stabilized methods, Comput. Methods Appl. Mech. Eng. 127 (1995) 387-401.

[44] T. J. R. Hughes, G. R. Feijóo, L. Mazzei, J.-B. Quincy, The variational multiscale method - a paradigm for computational mechanics, Comput. Methods Appl. Mech. Eng. 166 (1998) 3-24.

[45] T. J. R. Hughes, G. Scovazzi, F. L. P., Multiscale and stabilized methods, in: E. Stein, R. de Borst, T. J. R. Hughes (Eds.), Encyclopedia of Computational Mechanics, John Wiley \& Sons, 2004.

[46] F. Brezzi, M. Fortin, Mixed and Hybrid Finite Element Methods, volume 15 of Springer Series in Computational Mathematics, Springer, 1 edition, 1991

[47] T. J. R. Hughes, G. Sangalli, Variational multiscale analysis: The fine-scale Green's functions, projection, optimization, localization, and stabilized methods, SIAM J. Numer. Anal. 45 (2007) 539-557.

[48] T. Song, G. Scovazzi, A Nitsche method for wave propagation problems in time domain, Comput. Methods Appl. Mech. Eng. 293 (2015) $481-521$.

[49] G. Hauke, Simple stabilizing matrices for the computation of compressible flows in primitive variables, Comput. Methods Appl. Mech. Eng. 190 (2001) 6881-6893

[50] J. S. Wong, D. L. Darmofal, J. Peraire, The solution of the compressible Euler equations at low Mach numbers using a stabilized finite element algorithm, Comput. Methods Appl. Mech. Eng. 190 (2001) 5719-5737.

[51] J. V. Neumann, R. D. Richtmyer, A method for the numerical calculation of hydrodynamic shocks, J. Appl. Phys. 21 (1950) $232-237$.

[52] T. J. R. Hughes, G. Engel, L. Mazzei, M. G. Larson, The continuous Galerkin method is locally conservative, J. Comput. Phys. 163 (2000) 467-488.

[53] T. J. R. Hughes, G. N. Wells, Conservation properties for the Galerkin and stabilised forms of the advectiondiffusion and incompressible Navier-Stokes equations, Comput. Methods Appl. Mech. Eng. 194 (2005) 1141-1159.

[54] R. Löhner, C. Yang, Improved ALE mesh velocities for moving bodies, Commun. Numer. Meth. Eng. 12 (1996) 599-608.

[55] P. M. Knupp, Winslow smoothing on two-dimensional unstructured meshes, Eng. Comput. 15 (1999) $263-268$.

[56] J. L. F. Aymone, Mesh motion techniques for the ALE formulation in 3D large deformation problems, Int. J. Numer. Meth. Eng. 59 (2004) 1879-1908.

[57] A. A. Johnson, T. E. Tezduyar, Mesh update strategies in parrallel finite element computations of flow problems with moving boundaries and interfaces, Comput. Methods Appl. Mech. Eng. 119 (1994) 73-94.

[58] G. Olivier, F. Alauzet, A mesh topology change ALE framework for efficient body large-displacement adaptive simulations, in: J. C. F. Pereira, A. Sequeira (Eds.), V European Conference on Computational Fluid Dynamics. Lisbon, Portugal.

[59] B. Koobus, C. Farhat, Second-order time-accurate and geometrically conservative implicit schemes for flow computations on unstructured dynamic meshes, Comput. Methods Appl. Mech. Eng. 170 (1999) 103-129.

[60] C. Farhat, P. Geuzaine, C. Grandmont, The discrete geometric conservation law and the nonlinear stability of ALE schemes for the solution of flow problems on moving grids, J. Comput. Phys. 174 (2001) 669-694.

[61] J. H. Williamson, Low-storage Runge-Kutta schemes, J. Comput. Phys. 35 (1980) 48-56.

[62] R.-H. Ni, A multiple-grid scheme for solving the Euler equations, AIAA J. 20 (1982) 1565-1571.

[63] L. I. Sedov, Similarity and Dimensional Methods in Mechanics, CRC Press, 10th edition, 1993. 